Supporting Information 1

Experimental Section

\title{
Meldrum's Acids as Acylating Agents in the Catalytic Intramolecular Friedel-Crafts Reaction
}

\author{
Eric Fillion*, Dan Fishlock, Ashraf Wilsily, and Julie M. Goll \\ Department of Chemistry, University of Waterloo, Waterloo, Ontario N2L 3G1, Canada \\ efillion@uwaterloo.ca
}




\section{Table of Contents for Supporting Information 1}

General Methods

General Procedure A - Preparation of Mono-Benzylic Meldrum’s Acids via a One-Pot Reductive Alkylation 2

General Procedure B - Alkylation of 5-Substituted Meldrum's Acid and Dialkylation of Meldrum's Acid 3

General Procedure C - Conjugate Addition of Grignard Reagents to Meldrum's Alkylidenes 3

General Procedure D - Intramolecular Metal Trifluoromethanesulfonate - Catalyzed Friedel-Crafts Acylation of Meldrum's Acid Derivatives

Compound Specific Experimental Data 4

References

General Methods. All reactions were carried out in flame-dried glassware under a dry nitrogen atmosphere. Nitromethane and DMF were distilled from $\mathrm{CaH}_{2} . \mathrm{Sc}(\mathrm{OTf})_{3}$ was dried under high vacuum $(0.5 \mathrm{mmHg})$ for 2 hours at $180{ }^{\circ} \mathrm{C}$ and stored in a dry-box. ${ }^{1} \mathrm{H}$ NMR spectra were referenced to residual ${ }^{1} \mathrm{H}$ shift in $\mathrm{CDCl}_{3}(7.24 \mathrm{ppm})$. $\mathrm{CDCl}_{3}(77.0 \mathrm{ppm})$ was used as the internal reference for ${ }^{13} \mathrm{C}$ NMR spectra. Reactions were monitored by thin-layer chromatography (TLC) on commercial silica pre-coated plates with a particle size of $60 \AA$. Developed plates were viewed by UV lamp (254 nm), and with $p$-anisaldehyde stain. Flash chromatography was performed using 230-400 mesh silica gel. Melting points are uncorrected. Methyl $^{1}$, and dimethyl Meldrum's alkylidenes ${ }^{2}$ were prepared according to literature procedures. Cyclohexyl, tetrahydro-4-pyran, tetrahydro-4-thiopyran, and 4-cyclohexanone (ethylene ketal) Meldrum's alkylidenes were prepared from their respective ketones according to the method of Baty. ${ }^{3}$

\section{General Procedure A - Preparation of Mono-Benzylic Meldrum's Acids via a One-Pot} Reductive Alkylation: Meldrum's acid (1 eq) and the appropriate benzaldehyde (0.98 eq) were dissolved in absolute $\mathrm{EtOH}(0.5-1.0 \mathrm{M})$, followed by the addition of catalytic piperidinium acetate $(0.1 \mathrm{eq})$. The resulting solution was stirred vigorously for 30 minutes, and then cooled to $0{ }^{\circ} \mathrm{C} . \mathrm{NaBH}_{3} \mathrm{CN}$ (1.5 eq) was added portion-wise over 60 minutes, and the reaction allowed to warm to room temperature. The reaction was monitored by TLC and upon completion was carefully quenched with $10 \% \mathrm{HCl}$ (extreme caution should be exercised due to the evolution of HCN gas). Vigorous stirring was maintained until gas evolution had ceased, after which time the reaction was concentrated in vacuo to remove EtOH. The residue was re-suspended in $10 \% \mathrm{HCl}$ and extracted four times with $\mathrm{CH}_{2} \mathrm{Cl}_{2}$. The combined organic layers were 
dried over $\mathrm{MgSO}_{4}$, filtered, and concentrated. The resulting product was generally found to be of good purity by ${ }^{1} \mathrm{H} \mathrm{NMR}$, but was recrystallized from $\mathrm{MeOH}$ or purified by flash chromatography on silica gel.

General Procedure B - Alkylation of 5-Substituted Meldrum's Acid and Dialkylation of Meldrum's Acid: Equivalents are doubled for the dialkylation. Anhydrous $\mathrm{K}_{2} \mathrm{CO}_{3}(1.5 \mathrm{eq})$ was added to a solution of 5-substituted Meldrum's acid (1 eq) in dry DMF (1.0 M). To the vigorously stirred suspension was added dropwise the appropriate electrophile, such as iodomethane (5-10 eq), allyl bromide (1.2 eq), propargyl bromide (1.2 eq), or benzyl bromides (1.1 eq). The reaction was monitored by TLC (typical reaction times are 30-120 minutes for activated electrophiles and 16-24 hours for iodomethane). Upon completion, the mixture was poured into water (15 vol eq relative to DMF) and extracted 3 times with EtOAc. The combined organic layers were washed twice with saturated aqueous $\mathrm{NaHCO}_{3}$, brine, dried over $\mathrm{MgSO}_{4}$, filtered and concentrated. The resulting crude products were typically very pure by ${ }^{1} \mathrm{H}$ NMR and nearly quantitative in yield, but were purified by recrystallization or flash chromatography on silica gel.

General Procedure C - Conjugate Addition of Grignard Reagents to Meldrum's Alkylidenes: In a typical reaction, Meldrum's alkylidene was suspended in dry THF $(0.1 \mathrm{M})$ and cooled to $0{ }^{\circ} \mathrm{C}$ with stirring under inert atmosphere. A solution of the appropriate Grignard reagent (2 eq) in THF (or $\mathrm{Et}_{2} \mathrm{O}$ ) (0.5 to $3 \mathrm{M}$ ) was added dropwise over one hour, and then the reaction allowed to warm to room temperature and monitored by TLC. Upon completion, the reaction was quenched with $5 \% \mathrm{HCl}$ and extracted three times with EtOAc. The combined organic layers were washed with $\mathrm{H}_{2} \mathrm{O}$, brine, then dried over $\mathrm{MgSO}_{4}$ and filtered. Concentrated provided the crude product that was purified by either recrystallization or by flash chromatography on silica gel.

General Procedure D - Intramolecular Metal Trifluoromethanesulfonate - Catalyzed FriedelCrafts Acylation of Meldrum's Acid Derivatives: Reactions were typically performed on 200 milligrams of substrate. In a flame-dried round bottomed flask equipped with a magnetic stir bar, a reflux condenser and under inert atmosphere, was placed the substrate and $\mathrm{Sc}(\mathrm{OTf})_{3}(0.1 \mathrm{eq})$ (or other solid catalyst; liquid catalysts were administered to a solution of the substrate in $\mathrm{CH}_{3} \mathrm{NO}_{2}$ ). Distilled $\mathrm{CH}_{3} \mathrm{NO}_{2}$ was added in one portion by syringe, and the resulting suspension immediately placed into an oil bath preheated to $100{ }^{\circ} \mathrm{C}$. The reaction was maintained at this temperature and monitored by TLC until complete consumption of starting material was observed. The reaction was then concentrated under vacuum and directly subjected to flash chromatography, using a small quantity of $\mathrm{CH}_{2} \mathrm{Cl}_{2}$ to assist column loading. 
The slow (syringe pump) addition of a substrate solution to a stirring suspension of $\mathrm{Sc}(\mathrm{OTf})_{3}$ at $100{ }^{\circ} \mathrm{C}$ was found to be advantageous for less activated $\pi$-nucleophiles.

5,6-Dimethoxy-1-indanone ${ }^{4}$ (2): Prepared from 27 in 59\% yield using Procedure D in 45 min. Purified by flash chromatography (6:5 hexanes:EtOAc) to provide a white solid. M.p. $116-118{ }^{\circ} \mathrm{C}$ $\left(\mathrm{CH}_{2} \mathrm{Cl}_{2}\right)$. Lit. $119.5-120.5^{\circ} \mathrm{C}$ and $118.8-119.5{ }^{\circ} \mathrm{C} ;{ }^{1} \mathrm{H}$ NMR $\left(\mathrm{CDCl}_{3}, 300 \mathrm{MHz}\right) \delta 7.13(\mathrm{~s}, 1 \mathrm{H}), 6.85(\mathrm{~s}$, $1 \mathrm{H}), 3.92(\mathrm{~s}, 3 \mathrm{H}), 3.86(\mathrm{~s}, 3 \mathrm{H}), 3.03-2.99(\mathrm{~m}, 2 \mathrm{H}), 2.65-2.61(\mathrm{~m}, 2 \mathrm{H}) ;{ }^{13} \mathrm{C} \mathrm{NMR}\left(\mathrm{CDCl}_{3}, 75 \mathrm{MHz}\right) \delta$ 205.7, 155.3, 150.4, 149.3, 129.8, 107.4, 104.1, 56.1, 56.0, 36.4, 25.5; HRMS m/z calcd for $\mathrm{C}_{11} \mathrm{H}_{12} \mathrm{O}_{3}$ $\left(\mathrm{M}^{+}\right)$: 192.0786. Found: 192.0787.

Dimethyl 2-(3,5-dimethoxybenzyl)-2-methylmalonate (4): Compound (3) was alkylated according to Procedure B using iodomethane. Recrystallization from $\mathrm{MeOH}$ provided white crystals in $70 \%$ yield. M.p. $75-76{ }^{\circ} \mathrm{C}(\mathrm{MeOH}) ;{ }^{1} \mathrm{H} \mathrm{NMR}\left(\mathrm{CDCl}_{3}, 300 \mathrm{MHz}\right) \delta 6.28(\mathrm{~s}, 1 \mathrm{H}), 6.20$ (s, 2H), 3.69-3.68 (br s, 12H), $3.12(\mathrm{~s}, 2 \mathrm{H}), 1.31(\mathrm{~s}, 3 \mathrm{H}) ;{ }^{13} \mathrm{C} \mathrm{NMR}\left(\mathrm{CDCl}_{3}, 75 \mathrm{MHz}\right) \delta 172.1,160.4,138.0,108.1,98.6,55.0,54.6,52.3$, 41.3, 19.6; HRMS(EI) $m / z$ calcd for $\mathrm{C}_{15} \mathrm{H}_{20} \mathrm{O}_{6}\left(\mathrm{M}^{+}\right)$: 296.1260. Found: 296.1249.

5-(3,5-Dimethoxybenzyl)-2,2-dimethyl-1,3-dioxane-4,6-dione (9): Prepared by reductive alkylation of Meldrum's acid with 3,5-dimethoxybenzaldehyde using Procedure A. Recrystallization from $\mathrm{MeOH}$ provided a fine white solid in $61 \%$ yield. M.p. $102-103{ }^{\circ} \mathrm{C}(\mathrm{MeOH}) ;{ }^{1} \mathrm{H} \mathrm{NMR}\left(\mathrm{CDCl}_{3}, 300 \mathrm{MHz}\right)$ $\delta 6.44(\mathrm{~d}, J=2.3 \mathrm{~Hz}, 2 \mathrm{H}), 6.29(\operatorname{app~t}, J=2.2 \mathrm{~Hz}, 1 \mathrm{H}), 3.73(\mathrm{t}, J=4.9 \mathrm{~Hz}, 1 \mathrm{H}), 3.37(\mathrm{~d}, J=4.9 \mathrm{~Hz}, 2 \mathrm{H})$, $1.70(\mathrm{~s}, 3 \mathrm{H}), 1.51(\mathrm{~s}, 3 \mathrm{H}) ;{ }^{13} \mathrm{C} \mathrm{NMR}\left(\mathrm{CDCl}_{3}, 75 \mathrm{MHz}\right) \delta 165.2,160.7,139.6,107.4,105.1,99.1,55.2$, 47.9, 32.1, 28.4, 27.0; Anal. calcd for $\mathrm{C}_{15} \mathrm{H}_{18} \mathrm{O}_{6}$ : C, 61.22; H, 6.16. Found: C, 61.28; H, 6.26.

5,7-Dimethoxy-1-indanone 5 (10): Prepared from 9 in 73\% yield using Procedure D in 60 min. Purified by flash chromatography using 2:1 EtOAc:hexanes; M.p. 98-99 ${ }^{\circ} \mathrm{C}$ (benzene/petroleum ether). Lit. $98-99{ }^{\circ} \mathrm{C} ;{ }^{1} \mathrm{H} \mathrm{NMR}\left(\mathrm{CDCl}_{3}, 300 \mathrm{MHz}\right) \delta 6.42$ (br s, $\left.1 \mathrm{H}\right), 6.24$ (br s, $\left.1 \mathrm{H}\right), 3.85$ (s, 3H), 3.82 (s, 3H), 2.98-2.94 (m, 2H), 2.60-2.56 (m, 2H); ${ }^{13} \mathrm{C} \mathrm{NMR}\left(\mathrm{CDCl}_{3}, 75 \mathrm{MHz}\right) \delta 203.3,166.8,160.3,159.2,119.3$, 101.5, 97.3, 55.6, 36.8, 25.8. Anal. calcd for $\mathrm{C}_{11} \mathrm{H}_{12} \mathrm{O}_{3}$ : C, 68.74; H, 6.29. Found: C, 69.03; H, 6.30.

5-[1-(3,5-Dimethoxyphenyl)ethyl]-2,2-dimethyl-1,3-dioxane-4,6-dione (11): Prepared by conjugate addition of 3,5-dimethoxyphenylmagnesium chloride to methyl Meldrum's alkylidene using Procedure $\mathrm{C}$ in $49 \%$ yield after recrystallization from $\mathrm{MeOH}$. M.p. $111-112{ }^{\circ} \mathrm{C}(\mathrm{MeOH}) ;{ }^{1} \mathrm{H}$ NMR $\left(\mathrm{CDCl}_{3}, 300 \mathrm{MHz}\right) \delta 6.53-6.52(\mathrm{~m}, 2 \mathrm{H}), 6.36-6.31(\mathrm{~m}, 1 \mathrm{H}), 3.95(\mathrm{dq}, J=7.2,2.9 \mathrm{~Hz}, 1 \mathrm{H}), 3.78(\mathrm{~s}, 6 \mathrm{H})$, $3.69(\mathrm{~d}, J=3.0 \mathrm{~Hz}, 1 \mathrm{H}), 1.69(\mathrm{~s}, 3 \mathrm{H}), 1.64(\mathrm{~d}, J=7.3 \mathrm{~Hz}, 3 \mathrm{H}), 1.40(\mathrm{~s}, 3 \mathrm{H}) ;{ }^{13} \mathrm{C} \mathrm{NMR}\left(\mathrm{CDCl}_{3}, 75 \mathrm{MHz}\right)$ 
$\delta 164.9,164.8,160.8,143.5,106.4,105.2,99.4,55.3,52.4,39.7,28.2,27.9,17.8$; Anal. calcd for $\mathrm{C}_{16} \mathrm{H}_{20} \mathrm{O}_{6}: \mathrm{C}, 62.33 ; \mathrm{H}, 6.54$. Found: $\mathrm{C}, 62.05 ; \mathrm{H}, 6.61$.

5,7-Dimethoxy-3-methyl-1-indanone (12): Prepared from 11 in 77\% yield using Procedure D in 60 min. Purified by flash chromatography using 2:1 EtOAc:hexanes; M.p. 61-62.5 ${ }^{\circ} \mathrm{C} ;{ }^{1} \mathrm{H}$ NMR $\left(\mathrm{CDCl}_{3}, 300\right.$ MHz) $\delta 6.45$ (br s, 1H), 6.27 (br s, 1H), 3.87 (s, 3H), 3.85 (s, 3H), 3.28-3.22 (m, 1H), $2.85(\mathrm{dd}, J=18.5$, $7.6 \mathrm{~Hz}, 1 \mathrm{H}), 2.21(\mathrm{dd}, J=18.5,3.5 \mathrm{~Hz}, 1 \mathrm{H}), 1.32(\mathrm{~d}, J=7.6 \mathrm{~Hz}, 3 \mathrm{H}) ;{ }^{13} \mathrm{C} \mathrm{NMR}\left(\mathrm{CDCl}_{3}, 75 \mathrm{MHz}\right) \delta$ 202.4, 167.0, 165.2, 159.0, 118.6, 100.4, 97.2, 55.7, 55.6, 45.9, 32.5, 21.3. Anal. calcd for $\mathrm{C}_{12} \mathrm{H}_{14} \mathrm{O}_{3}: \mathrm{C}_{\text {, }}$ 69.88; H, 6.84. Found: C, 69.82; H, 6.73.

5-[1-(3,5-Dimethoxyphenyl)-1-methylethyl]-2,2-dimethyl-1,3-dioxane-4,6-dione (13): Prepared by conjugate addition of 3,5-dimethoxyphenylmagnesium chloride to dimethyl Meldrum's alkylidene using Procedure $\mathrm{C}$ to provide a 76\% yield of white solid after recrystallization from MeOH. M.p. 121-122 ${ }^{\circ} \mathrm{C}(\mathrm{MeOH}) ;{ }^{1} \mathrm{H}$ NMR $\left(\mathrm{CDCl}_{3}, 300 \mathrm{MHz}\right) \delta$ 6.46-6.45 (m, 2H), 6.33-6.31 (m, 1H), 3.75 (s, 6H), 3.59 (s, 1H), $1.63(\mathrm{~s}, 3 \mathrm{H}), 1.61(\mathrm{~s}, 6 \mathrm{H}), 1.34(\mathrm{~s}, 3 \mathrm{H}) ;{ }^{13} \mathrm{C} \mathrm{NMR}\left(\mathrm{CDCl}_{3}, 75 \mathrm{MHz}\right) \delta 164.0,160.6,147.4,105.0$, 104.8, 98.1, 57.1, 55.2, 42.5, 28.9, 27.6, 27.5; Anal. Calcd for $\mathrm{C}_{17} \mathrm{H}_{22} \mathrm{O}_{6}: \mathrm{C}, 63.34$; H, 6.88. Found: C, 63.22; H, 7.06.

5,7-Dimethoxy-3,3-dimethyl-1-indanone ${ }^{6}$ (14): Prepared from 13 in $82 \%$ yield using Procedure D with $\mathrm{CH}_{3} \mathrm{CN}$ in 120 min. Purified by flash chromatography using 2:1 EtOAc:hexanes; M.p. 92-94 ${ }^{\circ} \mathrm{C}$ (Et $\left.\mathrm{E}_{2} \mathrm{O}\right)$. Lit. $95.5{ }^{\circ} \mathrm{C}$ (benzene); ${ }^{1} \mathrm{H} \mathrm{NMR}\left(\mathrm{CDCl}_{3}, 300 \mathrm{MHz}\right) \delta 6.44$ (br s, 1H), 6.27 (br s, 1H), 3.89 (s, 3H), 3.87 (s, 3H), $2.52(\mathrm{~s}, 2 \mathrm{H}), 1.34(\mathrm{~s}, 6 \mathrm{H}) ;{ }^{13} \mathrm{C} \mathrm{NMR}\left(\mathrm{CDCl}_{3}, 75 \mathrm{MHz}\right) \delta 201.6,168.9,167.0,158.8$, 117.5, 98.8, 96.9, 55.7, 55.6, 53.4, 37.9, 29.7. Anal. calcd for $\mathrm{C}_{13} \mathrm{H}_{16} \mathrm{O}_{3}: \mathrm{C}, 70.89 ; \mathrm{H}, 7.32$. Found: C, $70.87 ; \mathrm{H}, 7.49$.

5-[1-(3,5-Dimethoxyphenyl)cyclohexyl]-2,2-dimethyl-1,3-dioxane-4,6-dione (15): Prepared by conjugate addition of 3,5-dimethoxyphenylmagnesium chloride to cyclohexyl Meldrum's alkylidene using Procedure $\mathrm{C}$ to provide a $78 \%$ yield of white solid after recrystallization from $\mathrm{MeOH}$. M.p. $124-125{ }^{\circ} \mathrm{C}$ $(\mathrm{MeOH}) ;{ }^{1} \mathrm{H}$ NMR $\left(\mathrm{CDCl}_{3}, 300 \mathrm{MHz}\right) \delta 6.38(\mathrm{~m}, 2 \mathrm{H}), 6.29(\mathrm{~m}, 1 \mathrm{H}), 3.60(\mathrm{~s}, 6 \mathrm{H}), 3.38(\mathrm{~s}, 1 \mathrm{H}), 2.38-2.33$ $(\mathrm{m}, 2 \mathrm{H}), 2.00-1.90(\mathrm{~m}, 2 \mathrm{H}), 1.70-1.50(\mathrm{~m}, 2 \mathrm{H}), 1.44(\mathrm{~s}, 3 \mathrm{H}), 1.44-1.30(\mathrm{~m}, 4 \mathrm{H}), 0.84(\mathrm{~s}, 3 \mathrm{H}) ;{ }^{13} \mathrm{C}$ NMR $\left(\mathrm{CDCl}_{3}, 75 \mathrm{MHz}\right) \delta 164.3,160.9,142.1,105.9,105.4,99.0,56.6,55.2,47.2,35.6,30.3,26.4,25.4,22.3$; Anal. Calcd for $\mathrm{C}_{20} \mathrm{H}_{26} \mathrm{O}_{6}$ : C, 66.28; H, 7.23. Found: C, 66.36; H, 7.33. 
5,7-Dimethoxy-(3.3)-pentamethylene-1-indanone (16): Prepared from 15 in 83\% yield using Procedure D with $\mathrm{CH}_{3} \mathrm{CN}$ in 120 min. Purified by flash chromatography using 1:1 EtOAc:hexanes; M.p. $132-133{ }^{\circ} \mathrm{C}\left(\mathrm{Et}_{2} \mathrm{O}\right) ;{ }^{1} \mathrm{H} \mathrm{NMR}\left(\mathrm{CDCl}_{3}, 300 \mathrm{MHz}\right) \delta 6.45(\mathrm{~d}, J=1.8 \mathrm{~Hz}, 1 \mathrm{H}), 6.26(\mathrm{~d}, J=1.8 \mathrm{~Hz}, 1 \mathrm{H}), 3.88$ (s, 3H), 3.86 (s, 3H), 2.51 (s, 2H), 1.76-1.19 (m, 10H); ${ }^{13} \mathrm{C} \mathrm{NMR}\left(\mathrm{CDCl}_{3}, 75 \mathrm{MHz}\right) \delta 201.9,169.3,166.9$, 159.0, 117.8, 99.3, 97.2, 55.8, 55.7, 49.1, 42.7, 38.2, 25.4, 23.6. Anal. calcd for $\mathrm{C}_{16} \mathrm{H}_{20} \mathrm{O}_{3}: \mathrm{C}, 73.82 ; \mathrm{H}$, 7.74. Found: C, 73.93; H, 7.83 .

\section{5-[4-(3,5-Dimethoxyphenyl)tetrahydro-2H-4-pyranyl]-2,2-dimethyl-1,3-dioxane-4,6-dione}

(17): Conjugate addition of 3,5-dimethoxyphenylmagnesium chloride to tetrahydro-4-pyran Meldrum's alkyidene using Procedure $\mathrm{C}$ provided a clear, colorless solid after recrystallization from $\mathrm{MeOH}$ in $73 \%$ yield. M.p. $147-148{ }^{\circ} \mathrm{C}(\mathrm{MeOH}) ;{ }^{1} \mathrm{H} \mathrm{NMR}\left(\mathrm{CDCl}_{3}, 300 \mathrm{MHz}\right) \delta 6.34$ (s, 3H), 3.84-3.77 (m, 2H), 3.71 (s, $6 \mathrm{H}), 3.60-3.47(\mathrm{~m}, 2 \mathrm{H}), 2.50-2.45(\mathrm{~m}, 2 \mathrm{H}), 2.30-2.22(\mathrm{~m}, 2 \mathrm{H}), 1.48(\mathrm{~s}, 3 \mathrm{H}), 0.86(\mathrm{~s}, 3 \mathrm{H}) ;{ }^{13} \mathrm{C}$ NMR $\left(\mathrm{CDCl}_{3}, 75 \mathrm{MHz}\right) \delta 163.9,161.3,140.9,105.8,105.7,99.4,64.0,55.6,55.3,45.3,36.0,30.2,26.7$; Anal. Calcd $\mathrm{C}_{15} \mathrm{H}_{18} \mathrm{O}_{6}$ : C, 62.63; H, 6.64. Found: C, 62.53; H, 6.71.

5,7-Dimethoxy-[3.3]-(3',4',6',7')tetrahydropyran-1-indanone (18): Prepared from 17 in $79 \%$ yield using Procedure D with $\mathrm{Yb}(\mathrm{OTf})_{3}$ in 15 min. Purified by flash chromatography (3:1 EtOAc:hexanes) to provide a white solid. M.p. 201-203 ${ }^{\circ} \mathrm{C}$ (EtOAc/hexanes); ${ }^{1} \mathrm{H}$ NMR $\left(\mathrm{CDCl}_{3}, 300 \mathrm{MHz}\right) \delta 6.47(\mathrm{~s}, 1 \mathrm{H})$, $6.28(\mathrm{~s}, 1 \mathrm{H}), 3.96(\mathrm{dd}, J=11.8,4.4 \mathrm{~Hz}, 2 \mathrm{H}), 3.86(\mathrm{~s}, 3 \mathrm{H}), 3.84(\mathrm{~s}, 3 \mathrm{H}), 3.48$ (br t, $J=12.3 \mathrm{~Hz}), 2.58(\mathrm{~s}$, 2H), $2.06(\mathrm{dt}, J=13.1,4.6 \mathrm{~Hz}, 2 \mathrm{H}), 1.36(\mathrm{brd}, J=13.4 \mathrm{~Hz}) ;{ }^{13} \mathrm{C} \mathrm{NMR}\left(\mathrm{CDCl}_{3}, 75 \mathrm{MHz}\right) \delta 200.4,167.2$, 167.1, 159.1, 117.8, 99.3, 97.5, 65.4, 55.8, 55.7, 48.4, 40.2, 38.0; $\mathrm{HRMS}(\mathrm{EI}) \mathrm{m} / \mathrm{z}$ calcd for $\mathrm{C}_{15} \mathrm{H}_{18} \mathrm{O}_{4}\left(\mathrm{M}^{+}\right)$: 262.1205. Found: 262.1209.

\section{5-[4-(3,5-Dimethoxyphenyl)tetrahydro-2H-4-thiopyranyl]-2,2-dimethyl-1,3-dioxane-4,6-dione}

(19): Conjugate addition of 3,5-dimethoxy phenylmagnesium chloride to tetrahydro-4-thiopyranyl Meldrum's alkyidene according to Procedure C produced a $69 \%$ yield of the desired product as a white powder after recrystallization from $\mathrm{MeOH}$. M.p. $135-136{ }^{\circ} \mathrm{C}(\mathrm{MeOH}) ;{ }^{1} \mathrm{H}$ NMR $\left(\mathrm{CDCl}_{3}, 300 \mathrm{MHz}\right)$ $\delta 6.33(\mathrm{~s}, 3 \mathrm{H}), 3.71(\mathrm{~s}, 6 \mathrm{H}), 3.41(\mathrm{~s}, 1 \mathrm{H}), 2.85-2.55(\mathrm{~m}, 6 \mathrm{H}), 2.50-2.30(\mathrm{~m}, 2 \mathrm{H}), 1.47$ (s, 3H), 0.89 (s, 3H); ${ }^{13} \mathrm{C} \mathrm{NMR}\left(\mathrm{CDCl}_{3}, 75 \mathrm{MHz}\right) \delta 163.8,161.2,140.5,106.0,105.7,99.2,56.4,55.3,46.3,36.5,30.3,26.5$, 24.1; $\mathrm{HRMS}(\mathrm{EI}) \mathrm{m} / \mathrm{z}$ calcd for $\mathrm{C}_{19} \mathrm{H}_{24} \mathrm{O}_{6} \mathrm{~S}\left(\mathrm{M}^{+}\right)$: 380.1293 . Found: 380.1302 .

5,7-Dimethoxy-[3.3]-(3',4',6',7')tetrahydrothiopyran-1-indanone (20): Prepared from 19 in $86 \%$ yield using Procedure D with $\mathrm{Yb}(\mathrm{OTf})_{3}$ in 20 min. Purified by flash chromatography (1:1 hexanes:EtOAc) SI-1-6 
to provide a white solid. M.p. $196-197{ }^{\circ} \mathrm{C}\left(\mathrm{CH}_{2} \mathrm{Cl}_{2}\right) ;{ }^{1} \mathrm{H} \mathrm{NMR}\left(\mathrm{CDCl}_{3}, 300 \mathrm{MHz}\right) \delta 6.49(\mathrm{~d}, J=1.8 \mathrm{~Hz}$, $1 \mathrm{H}), 6.29(\mathrm{~d}, J=1.8 \mathrm{~Hz}, 1 \mathrm{H}), 3.87(\mathrm{~s}, 3 \mathrm{H}), 3.86(\mathrm{~s}, 3 \mathrm{H}), 2.84-2.74(\mathrm{~m}, 2 \mathrm{H}), 2.59-2.54(\mathrm{~m}, 2 \mathrm{H}), 2.49$ (s, 2H), 2.10-2.00 (m, 2H), 1.79-1.72 (m, 2H); ${ }^{13} \mathrm{C} \mathrm{NMR}\left(\mathrm{CDCl}_{3}, 75 \mathrm{MHz}\right) \delta 200.4,167.9,167.1,159.2$, 117.5, 99.3, 97.7, 55.8, 47.9, 41.7, 38.4, 25.7; HRMS(EI) $m / z$ calcd for $\mathrm{C}_{15} \mathrm{H}_{18} \mathrm{O}_{3} \mathrm{~S}\left(\mathrm{M}^{+}\right)$: 278.0977 . Found: 278.0974.

\section{5-[8-(3,5-Dimethoxyphenyl)-1,4-dioxaspiro[4.5]dec-8-yl]-2,2-dimethyl-1,3-dioxane-4,6-dione}

(21): Conjugate addition of 3,5-dimethoxyphenylmagnesium chloride to cyclohexyl-4-one (ethylene ketal) Meldrum's alkylidene using Procedure C produced a 78\% yield following recrystallization from $\mathrm{MeOH}$. M.p. $139-140{ }^{\circ} \mathrm{C}(\mathrm{MeOH}) ;{ }^{1} \mathrm{H}$ NMR $\left(\mathrm{CDCl}_{3}, 300 \mathrm{MHz}\right) \delta$ 6.25-6.23 (m, 3H), 3.82-3.77 (m, $\left.4 \mathrm{H}\right), 3.63$ (s, $6 \mathrm{H}), 3.33(\mathrm{~s}, 1 \mathrm{H}), 2.50-2.40(\mathrm{~m}, 2 \mathrm{H}), 2.20-2.00(\mathrm{~m}, 2 \mathrm{H}), 1.70-1.40(\mathrm{~m}, 4 \mathrm{H}), 1.40(\mathrm{~s}, 3 \mathrm{H}), 0.78(\mathrm{~s}, 3 \mathrm{H}) ;{ }^{13} \mathrm{C}$ NMR $\left(\mathrm{CDCl}_{3}, 75 \mathrm{MHz}\right) \delta 164.0,160.9,140.6,107.6,105.8,105.4,99.1,64.0,55.7,55.1,46.0,32.7,31.1$, 30.0, 26.4; HRMS(EI) $m / z$ calcd for $\mathrm{C}_{22} \mathrm{H}_{28} \mathrm{O}_{8}\left(\mathrm{M}^{+}\right)$: 420.1784. Found: 420.1783.

5,7-Dimethoxy-[3.3]cyclohexan-6'-one (ethylene ketal)-1-indanone (22): Prepared from 21 in $78 \%$ yield using Procedure D with powdered $5 \AA$ molecular sieves ( 1 eq) and $\mathrm{Yb}(\mathrm{OTf})_{3}$ in 21 hours. Purified by flash chromatography $\left(4: 1\right.$ hexanes:EtOAc) to provide a pale yellow solid. M.p. $152-154{ }^{\circ} \mathrm{C}$ $\left(\mathrm{CH}_{2} \mathrm{Cl}_{2}\right) ;{ }^{1} \mathrm{H} \mathrm{NMR}\left(\mathrm{CDCl}_{3}, 300 \mathrm{MHz}\right) \delta 6.51(\mathrm{~d}, J=1.6 \mathrm{~Hz}, 1 \mathrm{H}), 6.29(\mathrm{~d}, J=1.6 \mathrm{~Hz}, 1 \mathrm{H}), 3.98(\mathrm{~d}, J=$ $1.7 \mathrm{~Hz}, 4 \mathrm{H}), 3.88(\mathrm{~d}, J=1.8 \mathrm{~Hz}, 3 \mathrm{H}), 3.86(\mathrm{~d}, J=1.6 \mathrm{~Hz}, 3 \mathrm{H}), 2.57(\mathrm{~d}, J=1.8 \mathrm{~Hz}, 2 \mathrm{H}), 2.09-2.05(\mathrm{~m}, 2 \mathrm{H})$, 1.82-1.61 (m, 4H), 1.56-1.51 (m, 2H) ${ }^{13} \mathrm{C}$ NMR $\left(\mathrm{CDCl}_{3}, 75 \mathrm{MHz}\right) \delta 201.1,168.1,167.1,159.1,118.0$, 108.0, 99.1, 97.8, 64.4, 64.3, 55.8, 48.3, 41.8, 35.8, 32.6; $\mathrm{HRMS}(\mathrm{EI}) \mathrm{m} / z$ calcd for $\mathrm{C}_{18} \mathrm{H}_{22} \mathrm{O}_{5}\left(\mathrm{M}^{+}\right)$: 318.1467. Found: 318.1463.

5-(3,5-Dimethylbenzyl)-2,2-dimethyl-1,3-dioxane-4,6-dione (23): Reductive alkylation of Meldrum's acid according to Procedure A with 3,5-dimethylbenzaldehyde provided a clear colorless oil after flash chromatography (62\%) which solidified on standing. M.p. $69-70{ }^{\circ} \mathrm{C}(\mathrm{MeOH}) ;{ }^{1} \mathrm{H}$ NMR $\left(\mathrm{CDCl}_{3}, 300 \mathrm{MHz}\right) \delta 6.91$ (br s, 2H), 6.85 (br s, 1H), 3.72 (t, $\left.J=4.9 \mathrm{~Hz}, 1 \mathrm{H}\right), 3.39$ (d, J= 4.9 Hz, 2H), $2.60(\mathrm{~s}, 6 \mathrm{H}), 1.72(\mathrm{~s}, 3 \mathrm{H}), 1.48(\mathrm{~s}, 3 \mathrm{H}) ;{ }^{13} \mathrm{C} \mathrm{NMR}\left(\mathrm{CDCl}_{3}, 75 \mathrm{MHz}\right) \delta 165.4,138.1,137.2,128.7,127.3$, 105.2, 48.2, 31.9, 28.4, 27.1, 21.2; HRMS(EI) $m / z$ calcd for $\mathrm{C}_{15} \mathrm{H}_{18} \mathrm{O}_{4}\left(\mathrm{M}^{+}\right)$: 262.1205. Found: 262.1206.

5,7-Dimethyl-1-indanone ${ }^{7}$ (24): Prepared from 23 in 52\% yield following Procedure D using the slow addition of substrate over 9.5 h. Purified by flash chromatography using 8:1 hexanes:EtOAc; M.p. 75-76 ${ }^{\circ} \mathrm{C}(\mathrm{MeOH})$. Lit. 76-77 ${ }^{\circ} \mathrm{C} ;{ }^{1} \mathrm{H}$ NMR $\left(\mathrm{CDCl}_{3}, 300 \mathrm{MHz}\right) \delta 7.05$ (s, 1H), $6.89(\mathrm{~s}, 1 \mathrm{H}), 3.02-2.98$ (m, 
2H), 2.64-2.60 (m, 2H), $2.57(\mathrm{~s}, 3 \mathrm{H}), 2.36(\mathrm{~s}, 3 \mathrm{H}) ;{ }^{13} \mathrm{C} \mathrm{NMR}\left(\mathrm{CDCl}_{3}, 75 \mathrm{MHz}\right) \delta 207.5,156.5,145.0$, 138.5, 132.2, 130.3, 124.4, 36.1, 25.2, 21.8, 18.2. Anal. calcd for $\mathrm{C}_{11} \mathrm{H}_{12} \mathrm{O}: \mathrm{C}, 82.46 ; \mathrm{H}, 7.55$. Found: $\mathrm{C}$, 82.40; H, 7.36.

5-[1-(3,5-Dimethylphenyl)cyclohexyl]-2,2-dimethyl-1,3-dioxane-4,6-dione (25): Prepared by conjugate addition of 3,5-dimethylphenylmagnesium bromide to cyclohexyl Meldrum's alkylidene according to Procedure $\mathrm{C}$, and recrystallized using $\mathrm{MeOH}$ to provide a white solid in $88 \%$ yield. M.p. 144.5-146 ${ }^{\circ} \mathrm{C}(\mathrm{MeOH}) ;{ }^{1} \mathrm{H} \mathrm{NMR}\left(\mathrm{CDCl}_{3}, 300 \mathrm{MHz}\right) \delta 6.87$ (br s, 3H), 3.42 (s, 1H), 2.46-2.41 (m, 2H), $2.26(\mathrm{~s}, 6 \mathrm{H}), 2.04-1.96(\mathrm{~m}, 2 \mathrm{H}), 2.75-2.60(\mathrm{~m}, 2 \mathrm{H}), 1.46(\mathrm{~s}, 3 \mathrm{H}), 1.46-1.40(\mathrm{~m}, 4 \mathrm{H}), 0.71(\mathrm{~s}, 3 \mathrm{H}) ;{ }^{13} \mathrm{C}$ $\operatorname{NMR}\left(\mathrm{CDCl}_{3}, 75 \mathrm{MHz}\right) \delta 164.2,139.5,137.8,128.6,125.1,105.4,56.5,46.7,38.4,30.3,25.8,25.5,22.1$, 21.2; Anal. Calcd for $\mathrm{C}_{20} \mathrm{H}_{26} \mathrm{O}_{4}$ : C, 72.70; H, 7.93. Found: C, 72.65; H, 7.89.

5,7-Dimethyl-(3.3)-pentamethylene-1-indanone (26): Prepared from 25 in $75 \%$ yield using Procedure D in 90 min. Purified by flash chromatography using 10:1 hexanes:EtOAc; M.p. 87.5-88.5 ${ }^{\circ} \mathrm{C}$ (hexanes); ${ }^{1} \mathrm{H}$ NMR $\left(\mathrm{CDCl}_{3}, 300 \mathrm{MHz}\right) \delta 7.08$ (s, 1H), 6.89 (s, 1H), 2.57 (s, 3H), 2.52 (s, 2H), 2.37 (s, 3H), 1.77-1.23 (m, 10H); ${ }^{13} \mathrm{C}$ NMR $\left(\mathrm{CDCl}_{3}, 75 \mathrm{MHz}\right) \delta 206.5,165.6,145.0,138.2,130.5,121.7,49.1$, 42.1, 38.4, 25.6, 23.8, 22.0, 18.3. Anal. calcd for $\mathrm{C}_{16} \mathrm{H}_{20} \mathrm{O}: \mathrm{C}, 84.16 ; \mathrm{H}, 8.83$. Found: $\mathrm{C}, 84.00 ; \mathrm{H}, 9.12$.

5-(3,4-Dimethoxybenzyl)-2,2-dimethyl-1,3-dioxane-4,6-dione ${ }^{8}$ (27): Prepared by reductive alkylation of Meldrum's acid with 3,4-dimethoxybenzaldehyde according to Procedure A. A fine white powder was obtained in $77 \%$ yield after recrystallizaton from $\mathrm{MeOH}$. M.p. 136-137 ${ }^{\circ} \mathrm{C}(\mathrm{MeOH})$. Lit. 142$144{ }^{\circ} \mathrm{C} ;{ }^{1} \mathrm{H}$ NMR $\left(\mathrm{CDCl}_{3}, 300 \mathrm{MHz}\right) \delta$ 6.77-6.64 (m, 3H), 3.75-3.73 (br s, 7H), 3.32 (d, J = 4.8 Hz, 2H), $1.63(\mathrm{~s}, 3 \mathrm{H}), 1.40(\mathrm{~s}, 3 \mathrm{H}) ;{ }^{13} \mathrm{C} \mathrm{NMR}\left(\mathrm{CDCl}_{3}, 75 \mathrm{MHz}\right) \delta 165.3,148.4,147.7,129.3,121.7,112.8,110.8$, 104.9, 55.5, 47.9, 31.5, 28.1, 26.9; $\mathrm{HRMS}(\mathrm{EI}) \mathrm{m} / \mathrm{z}$ calcd for $\mathrm{C}_{15} \mathrm{H}_{18} \mathrm{O}_{6}\left(\mathrm{M}^{+}\right)$: 294.1103. Found: 294.1106.

5-[1-(3,4-Dimethoxyphenyl)ethyl]-2,2-dimethyl-1,3-dioxane-4,6-dione (28): Conjugate addition of methylmagnesium iodide to 3,4-dimethoxyphenyl Meldrum's alkylidene (prepared by condensation of 3,4-dimethoxybenzaldehyde with Meldrum's acid) ${ }^{9}$ according to Procedure C produced a 53\% yield of a white powder after recrystallization from $\mathrm{MeOH}$. M.p. $137-138{ }^{\circ} \mathrm{C}(\mathrm{MeOH}) ;{ }^{1} \mathrm{H} \mathrm{NMR}\left(\mathrm{CDCl}_{3}, 300 \mathrm{MHz}\right)$ $\delta$ 6.82-6.79 (m, 2H), 6.72-6.69 (m, 1H), $3.87(\mathrm{dq}, J=7.3,3.0 \mathrm{~Hz}, 1 \mathrm{H}), 3.78(\mathrm{~s}, 3 \mathrm{H}), 3.76(\mathrm{~s}, 3 \mathrm{H}), 3.63(\mathrm{~d}, J$ $=3.0 \mathrm{~Hz}, 1 \mathrm{H}), 1.59(\mathrm{~s}, 6 \mathrm{H}), 1.26(\mathrm{~s}, 3 \mathrm{H}) ;{ }^{13} \mathrm{C} \mathrm{NMR}\left(\mathrm{CDCl}_{3}, 75 \mathrm{MHz}\right) \delta 165.2,164.7,148.5,148.0,133.3$, 120.3, 111.4, 110.7, 105.0, 55.6, 55.3, 39.2, 27.9, 27.7, 18.2; $\mathrm{HRMS}(\mathrm{EI}) \mathrm{m} / z$ calcd for $\mathrm{C}_{16} \mathrm{H}_{20} \mathrm{O}_{6}\left(\mathrm{M}^{+}\right)$: 308.1260. Found: 308.1269. 
5,6-Dimethoxy-3-methyl-1-indanone ${ }^{4}$ (29): Prepared from 28 in 68\% yield using Procedure D in 20 min. Purified by flash chromatography (4:1 hexanes:EtOAc) to provide a white solid. M.p. $87-88{ }^{\circ} \mathrm{C}$ (hexanes/EtOAc). Lit. 90.5-92 ${ }^{\circ} \mathrm{C} ;{ }^{1} \mathrm{H}$ NMR $\left(\mathrm{CDCl}_{3}, 300 \mathrm{MHz}\right) \delta 7.12$ (s, 1H), $6.86(\mathrm{~s}, 1 \mathrm{H}), 3.95$ (s, 3H), $3.88(\mathrm{~s}, 3 \mathrm{H}), 3.36-3.30(\mathrm{~m}, 1 \mathrm{H}), 2.92(\mathrm{dd}, J=7.2,1.2 \mathrm{~Hz}, 1 \mathrm{H}), 2.23(\mathrm{dd}, J=18.8,1.4 \mathrm{~Hz}, 1 \mathrm{H}), 1.35(\mathrm{~d}, J=$ $7.1 \mathrm{~Hz}, 3 \mathrm{H}) ;{ }^{13} \mathrm{C} \mathrm{NMR}\left(\mathrm{CDCl}_{3}, 75 \mathrm{MHz}\right) \delta 204.7,155.4,155.2,149.3,129.1,106.0,103.8,56.1,56.0$, 45.4, 32.4, 21.3; $\mathrm{HRMS}(\mathrm{EI}) \mathrm{m} / \mathrm{z}$ calcd for $\mathrm{C}_{12} \mathrm{H}_{14} \mathrm{O}_{3}\left(\mathrm{M}^{+}\right)$: 206.0943. Found: 206.0949 .

5-[1-(3,4-Dimethoxyphenyl)-1-methylethyl]-2,2-dimethyl-1,3-dioxane-4,6-dione (30): Conjugate addition of 3,4-dimethoxyphenylmagnesium bromide to dimethyl Meldrum's alkylidene according to Procedure $\mathrm{C}$ provided a white solid in $49 \%$ yield after recrystallization from EtOH. M.p. 137$138{ }^{\circ} \mathrm{C}(\mathrm{EtOH}) ;{ }^{1} \mathrm{H}$ NMR $\left(\mathrm{CDCl}_{3}, 300 \mathrm{MHz}\right) \delta$ 6.86-6.75 (m, 3H), $3.83(\mathrm{~s}, 3 \mathrm{H}), 3.81(\mathrm{~s}, 3 \mathrm{H}), 3.46(\mathrm{~s}, 1 \mathrm{H})$, $1.63(\mathrm{~s}, 6 \mathrm{H}), 1.56(\mathrm{~s}, 3 \mathrm{H}), 1.19(\mathrm{~s}, 3 \mathrm{H}) ;{ }^{13} \mathrm{C} \mathrm{NMR}\left(\mathrm{CDCl}_{3}, 75 \mathrm{MHz}\right) \delta 164.4,148.5,148.0,136.5,118.7$, 110.7, 110.1, 105.2, 57.7, 55.9, 55.8, 42.7, 29.5, 28.2, 27.2; Anal. Calcd for $\mathrm{C}_{17} \mathrm{H}_{22} \mathrm{O}_{6}$ : C, 63.34; H, 6.88. Found: C, 63.69; H, 6.93.

5,6-Dimethoxy-3,3-dimethyl-1-indanone ${ }^{4}$ (31): Prepared from 30 in 69\% yield using Procedure D in 20 min. Purified by flash chromatography (4:1 hexanes:EtOAc) to provide a pale yellow oil. ${ }^{1} \mathrm{H}$ NMR $\left(\mathrm{CDCl}_{3}, 300 \mathrm{MHz}\right) \delta 7.14(\mathrm{~s}, 1 \mathrm{H}), 6.87(\mathrm{~s}, 1 \mathrm{H}), 4.01(\mathrm{~s}, 3 \mathrm{H}), 3.92(\mathrm{~s}, 3 \mathrm{H}), 2.58(\mathrm{~s}, 2 \mathrm{H}), 1.42(\mathrm{~s}, 6 \mathrm{H}) ;{ }^{13} \mathrm{C}$ $\mathrm{NMR}\left(\mathrm{CDCl}_{3}, 75 \mathrm{MHz}\right) \delta 204.4,159.2,155.6,149.4,128.1,104.3,103.8,56.2,56.1,52.9,38.2,29.9$; HRMS(EI) $m / z$ calcd for $\mathrm{C}_{13} \mathrm{H}_{16} \mathrm{O}_{3}\left(\mathrm{M}^{+}\right)$: 220.1099. Found: 220.1108 .

5-(2,5-Dimethoxybenzyl)-2,2-dimethyl-1,3-dioxane-4,6-dione (32): Prepared by the reductive alkyation of Meldrum's acid with 2,5-dimethoxybenzaldehyde according to Procedure A, and recrystallized from $\mathrm{MeOH}$ to give a yield of 57\%. M.p. $94-95{ }^{\circ} \mathrm{C}(\mathrm{MeOH}) ;{ }^{1} \mathrm{H} \mathrm{NMR}\left(\mathrm{CDCl}_{3}, 300 \mathrm{MHz}\right) \delta 6.91(\mathrm{~s}, 1 \mathrm{H})$, $6.74(\mathrm{~d}, J=2.2 \mathrm{~Hz}, 2 \mathrm{H}), 4.00(\mathrm{t}, J=5.8 \mathrm{~Hz}, 1 \mathrm{H}), 3.75(\mathrm{~s}, 3 \mathrm{H}), 3.74(\mathrm{~s}, 3 \mathrm{H}), 3.34(\mathrm{~d}, J=5.8 \mathrm{~Hz}, 2 \mathrm{H}), 1.75$ (s, 3H), $1.70(\mathrm{~s}, 3 \mathrm{H}) ;{ }^{13} \mathrm{C} \mathrm{NMR}\left(\mathrm{CDCl}_{3}, 75 \mathrm{MHz}\right) \delta 165.3,153.3,151.3,126.7,117.8,112.5,104.8,60.4$, 55.7, 46.1, 28.6, 28.0, 26.4; $\mathrm{HRMS}(\mathrm{EI}) \mathrm{m} / \mathrm{z}$ calcd for $\mathrm{C}_{15} \mathrm{H}_{18} \mathrm{O}_{6}\left(\mathrm{M}^{+}\right)$: 294.1103. Found: 294.1095.

4,7-Dimethoxy-1-indanone ${ }^{10}$ (33): Prepared from 32 in 32\% yield using Procedure D in 20 min. Purified by flash chromatography $\left(1: 1\right.$ hexanes:EtOAc) to provide a white solid. M.p. $122-123{ }^{\circ} \mathrm{C}\left(\mathrm{Et}_{2} \mathrm{O}\right)$. Lit. $125-126{ }^{\circ} \mathrm{C} ;{ }^{1} \mathrm{H}$ NMR $\left(\mathrm{CDCl}_{3}, 300 \mathrm{MHz}\right) \delta 6.96(\mathrm{~d}, J=8.7 \mathrm{~Hz}, 1 \mathrm{H}), 6.70(\mathrm{~d}, J=8.7 \mathrm{~Hz}, 1 \mathrm{H}), 3.88(\mathrm{~s}$, 3H), $2.96(\mathrm{AB} \mathrm{m}, 2 \mathrm{H}), 2.65(\mathrm{AB} \mathrm{m}, 2 \mathrm{H}) ;{ }^{13} \mathrm{C} \mathrm{NMR}\left(\mathrm{CDCl}_{3}, 75 \mathrm{MHz}\right) \delta 204.3,151.8,150.4,145.9,126.3$, 116.5, 109.4, 55.9, 36.7, 22.2; $\mathrm{HRMS}(\mathrm{EI}) \mathrm{m} / \mathrm{z}$ for $\mathrm{C}_{17} \mathrm{H}_{17} \mathrm{NO}_{3}\left(\mathrm{M}^{+}\right)$: 192.0786. Found: 192.0782. 


\section{5-(2-[1-(tert-Butyl)-1,1-diphenylsilyl]oxy-5-methoxybenzyl)-2,2-dimethyl-1,3-dioxane-4,6-}

dione (34): Obtained by reductive alkylation of Meldrum's acid with TBDPS-protected 4methoxysalicylaldehydeaccording to Procedure A. Flash chromatography (10:3 hexanes:EtOAc), followed by recrystallization from $\mathrm{MeOH}$ gave a white solid in $21 \%$ yield. M.p. $128-129{ }^{\circ} \mathrm{C}(\mathrm{MeOH})$; ${ }^{1} \mathrm{H}$ NMR $\left(\mathrm{CDCl}_{3}, 300 \mathrm{MHz}\right) \delta 7.71-6.80(\mathrm{~m}, 4 \mathrm{H}), 7.46-7.35(\mathrm{~m}, 6 \mathrm{H}), 7.01($ br s, $1 \mathrm{H}), 6.38-6.36(\mathrm{~m}, 2 \mathrm{H}), 4.49(\mathrm{t}, J=$ $6.0 \mathrm{~Hz}, 1 \mathrm{H}), 3.69(\mathrm{~s}, 3 \mathrm{H}), 3.54(\mathrm{~d}, J=6.0 \mathrm{~Hz}, 2 \mathrm{H}), 1.73(\mathrm{~s}, 3 \mathrm{H}), 1.62(\mathrm{~s}, 3 \mathrm{H}), 1.10(\mathrm{~s}, 9 \mathrm{H}) ;{ }^{13} \mathrm{C} \mathrm{NMR}$ $\left(\mathrm{CDCl}_{3}, 75 \mathrm{MHz}\right) \delta 164.9,153.3,147.0,135.1,132.5,130.0,128.1,127.9,118.9,117.7,112.4,124.8$, 55.4, 46.7, 48.6, 27.7, 26.5, 25.6, 19.3; HRMS(EI) $m / z$ calcd for $\mathrm{C}_{30} \mathrm{H}_{34} \mathrm{O}_{6} \mathrm{Si}\left(\mathrm{M}^{+}\right)$: 518.2125. Found: 518.2142.

4-[1-(tert-Butyl)-1,1-diphenylsilyl]oxy-7-methoxy-1-indanone (35): Prepared from 34 in $63 \%$ yield using Procedure D in 35 min. Purified by flash chromatography (3:1 hexanes:EtOAc) to provide a pale yellow oil. ${ }^{1} \mathrm{H}$ NMR $\left(\mathrm{CDCl}_{3}, 300 \mathrm{MHz}\right) \delta 7.72(\mathrm{dd}, J=7.9,1.4 \mathrm{~Hz}, 4 \mathrm{H}), 7.42-7.31(\mathrm{~m}, 6 \mathrm{H}), 6.64(\mathrm{~d}, J$ $=6.6 \mathrm{~Hz}, 1 \mathrm{H}), 6.41(\mathrm{~d}, J=8.8 \mathrm{~Hz}, 1 \mathrm{H}), 3.82(\mathrm{~s}, 3 \mathrm{H}), 3.11(\mathrm{t}, J=5.8 \mathrm{~Hz}, 2 \mathrm{H}), 2.70(\mathrm{t}, J=5.9 \mathrm{~Hz}, 1 \mathrm{H}), 1.15$ (s, 9H); ${ }^{13} \mathrm{C} \mathrm{NMR}\left(\mathrm{CDCl}_{3}, 75 \mathrm{MHz}\right) \delta 205.0,152.1,147.3,146.1,135.4,132.5,130.1,127.9,126.1,125.0$, 109.4, 55.8, 36.8, 26.5, 22.8, 19.5; $\mathrm{HRMS}(\mathrm{EI}) \mathrm{m} / \mathrm{z}$ calcd for $\mathrm{C}_{26} \mathrm{H}_{28} \mathrm{O}_{3} \mathrm{Si}\left(\mathrm{M}^{+}\right)$: 416.1808. Found: 416.1799.

5-(2,3-Dimethoxybenzyl)-2,2-dimethyl-1,3-dioxane-4,6-dione (36): Prepared by the reductive alkylation of Meldrum's acid with veratraldehyde according to Procedure A. Recrystallization from $\mathrm{MeOH}$ provided white crystals in $44 \%$ yield. M.p. $120-121{ }^{\circ} \mathrm{C}(\mathrm{MeOH}) ;{ }^{1} \mathrm{H} \mathrm{NMR}\left(\mathrm{CDCl}_{3}, 300 \mathrm{MHz}\right) \delta 7.00-$ $6.95(\mathrm{~m}, 2 \mathrm{H}), 6.80(\mathrm{dd}, J=7.5,2.1 \mathrm{~Hz}, 1 \mathrm{H}), 4.11(\mathrm{t}, J=5.6 \mathrm{~Hz}, 1 \mathrm{H}), 3.84(\mathrm{~s}, 3 \mathrm{H}), 3.83(\mathrm{~s}, 3 \mathrm{H}), 3.34(\mathrm{~d}, J=$ $5.6 \mathrm{~Hz}, 2 \mathrm{H}), 1.75(\mathrm{~s}, 3 \mathrm{H}), 1.71$ (s, 3H); ${ }^{13} \mathrm{C} \mathrm{NMR}\left(\mathrm{CDCl}_{3}, 75 \mathrm{MHz}\right) \delta 165.2,152.4,146.9,131.8,123.9$, 123.4, 111.2, 104.9, 60.4, 55.6, 47.1, 29.6, 27.4, 26.2; Anal. Calcd for $\mathrm{C}_{15} \mathrm{H}_{18} \mathrm{O}_{6}$ : C, 61.22; H, 6.16. Found: C, 61.26; H, 6.17; HRMS(EI) $m / z$ calcd for $\mathrm{C}_{15} \mathrm{H}_{18} \mathrm{O}_{6}\left(\mathrm{M}^{+}\right)$: 294.1103. Found: 294.1109.

4,5-Dimethoxy-1-indanone ${ }^{11}$ (37): Prepared from 36 in 64\% yield using Procedure D in 80 min. Purified by flash chromatography $\left(1: 1\right.$ hexanes:EtOAc) to provide a pale yellow solid. M.p. $68-70{ }^{\circ} \mathrm{C}$ $\left(\mathrm{Et}_{2} \mathrm{O}\right)$. Lit. $74{ }^{\circ} \mathrm{C} ;{ }^{1} \mathrm{H} \mathrm{NMR}\left(\mathrm{CDCl}_{3}, 300 \mathrm{MHz}\right) \delta 7.46(\mathrm{~d}, J=8.3 \mathrm{~Hz}, 1 \mathrm{H}), 6.92(\mathrm{~d}, J=8.4 \mathrm{~Hz}, 1 \mathrm{H}), 3.90$ $(\mathrm{s}, 3 \mathrm{H}), 3.87(\mathrm{~s}, 3 \mathrm{H}), 3.07-3.02(\mathrm{AB} \mathrm{m}, 2 \mathrm{H}), 2.63-2.59(\mathrm{AB} \mathrm{m}, 2 \mathrm{H}) ;{ }^{13} \mathrm{C} \mathrm{NMR}\left(\mathrm{CDCl}_{3}, 75 \mathrm{MHz}\right) \delta 205.4$, 157.4, 147.8, 145.9, 131.0, 120.1, 112.2, 60.2, 56.1, 36.3, 22.4; HRMS(EI) $m / z$ calcd for $\mathrm{C}_{11} \mathrm{H}_{12} \mathrm{O}_{3}\left(\mathrm{M}^{+}\right)$: 192.0786. Found: 192.0782. 
5-[1-(2,3-Dimethoxyphenyl)ethyl]-2,2-dimethyl-1,3-dioxane-4,6-dione (38): The target material was obtained by the conjugate addition of $\mathrm{Me}_{2} \mathrm{AlCl}$ to 2,3-dimethoxyphenyl Meldrum's alkylidene (prepared by condensing 2,3-dimethoxybenzaldehyde with Meldrum's acid) ${ }^{9}$. A white powder was obtained in 51\% yield after flash chromatography (3:1 hexanes:EtOAc) and recrystallization from $\mathrm{MeOH}$. M.p. $123-123.5{ }^{\circ} \mathrm{C}(\mathrm{MeOH}) ;{ }^{1} \mathrm{H}$ NMR $\left(\mathrm{CDCl}_{3}, 300 \mathrm{MHz}\right) \delta$ 7.08-7.03 (m, 2H), 6.86-6.82 (m, 1H), 4.29 $(\mathrm{d}, J=2.9 \mathrm{~Hz}, 1 \mathrm{H}), 4.12(\mathrm{dq}, J=7.1,2.9 \mathrm{~Hz}, 1 \mathrm{H}), 3.86(\mathrm{~s}, 3 \mathrm{H}), 3.85$ (s, 3H), 1.69 (s, 3H), 1.67 (s, 3H), $1.43(\mathrm{~d}, J=7.1 \mathrm{~Hz}, 3 \mathrm{H}) ;{ }^{13} \mathrm{C} \mathrm{NMR}\left(\mathrm{CDCl}_{3}, 75 \mathrm{MHz}\right) \delta 165.5,163.5,152.1,146.3,135.6,123.7,120.9$, $110.9,104.6,60.6,55.6,50.8,31.6,28.3,26.7,13.9 ; \mathrm{HRMS}(\mathrm{EI}) \mathrm{m} / z$ calcd for $\mathrm{C}_{16} \mathrm{H}_{20} \mathrm{O}_{6}\left(\mathrm{M}^{+}\right)$: 308.1260. Found: 308.1251.

4,5-Dimethoxy-3-methyl-1-indanone (39): Prepared from 38 in 38\% yield using Procedure D in 40 min. Purified by flash chromatography (3:1 hexanes:EtOAc) to provide a clear colorless oil. ${ }^{1} \mathrm{H}$ NMR $\left(\mathrm{CDCl}_{3}, 300 \mathrm{MHz}\right) \delta 7.45(\mathrm{~d}, J=8.4 \mathrm{~Hz}), 6.93(\mathrm{~d}, J=8.4 \mathrm{~Hz}), 3.91(\mathrm{~s}, 3 \mathrm{H}), 3.89(\mathrm{~s}, 3 \mathrm{H}), 3.51(\mathrm{ddq}, J=$ 7.7, 7.0, 2.6 Hz, 1H), 2.87 (dd, $J=18.9,7.7 \mathrm{~Hz}, 1 \mathrm{H}), 2.22(\mathrm{dd}, J=18.9,2.6 \mathrm{~Hz}, 1 \mathrm{H}), 1.38(\mathrm{~d}, 7.0 \mathrm{~Hz}, 3 \mathrm{H})$; ${ }^{13} \mathrm{C} \mathrm{NMR}\left(\mathrm{CDCl}_{3}, 75 \mathrm{MHz}\right) \delta 205.0,158.0,152.3,145.8,130.6,119.9,112.4,60.5,56.1,45.8,30.8,21.0$; HRMS(EI) $m / z$ calcd for $\mathrm{C}_{12} \mathrm{H}_{14} \mathrm{O}_{3}\left(\mathrm{M}^{+}\right)$: 206.0943. Found: 206.0937.

\section{5-[1-(2,3-Dimethoxyphenyl)-1-methylethyl]-2,2-dimethyl-1,3-dioxane-4,6-dione (40):}

Conjugate addition of 2,3-dimethoxyphenylmagnesium bromide to dimethyl Meldrum's alkylidene according to Procedure C provided a $25 \%$ yield of white solid after flash chromatography (6:1 hexanes:EtOAc). M.p. 118-119 ${ }^{\circ} \mathrm{C}\left(\mathrm{CH}_{2} \mathrm{Cl}_{2}\right) ;{ }^{1} \mathrm{H} \mathrm{NMR}\left(\mathrm{CDCl}_{3}, 300 \mathrm{MHz}\right) \delta$ 7.02-6.94 (m, $\left.2 \mathrm{H}\right), 6.81$ (dd, $J=7.2,2.3 \mathrm{~Hz}, 1 \mathrm{H}), 5.38(\mathrm{~s}, 1 \mathrm{H}), 3.89(\mathrm{~s}, 3 \mathrm{H}), 3.80(\mathrm{~s}, 3 \mathrm{H}), 1.77(\mathrm{~s}, 3 \mathrm{H}), 1.65(\mathrm{~s}, 3 \mathrm{H}), 1.63(\mathrm{~s}, 6 \mathrm{H}) ;{ }^{13} \mathrm{C}$ $\operatorname{NMR}\left(\mathrm{CDCl}_{3}, 75 \mathrm{MHz}\right) \delta 163.6,152.3,146.4,140.2,123.1,118.7,110.7,103.6,60.4,55.3,53.5,39.5$, 28.4, 25.9, 25.5; HRMS(EI) $m / z$ calcd for $\mathrm{C}_{17} \mathrm{H}_{22} \mathrm{O}_{6}\left(\mathrm{M}^{+}\right)$: 322.1416. Found: 322.1419.

\section{5-(2-[1-(tert-Butyl)-1,1-diphenylsilyl]oxy-3-methoxybenzyl)-2,2-dimethyl-1,3-dioxane-4,6-}

dione (42): Reductive alkylation of Meldrum's acid with TBDPS-protected $o$-vanillin according to Procedure A, followed by flash chromatography (8:1 hexanes:EtOAc) gave a 32\% yield of white solid. M.p. $116-118{ }^{\circ} \mathrm{C}$ (Benzene); ${ }^{1} \mathrm{H}$ NMR $\left(\mathrm{CDCl}_{3}, 300 \mathrm{MHz}\right) \delta 7.63(\mathrm{dd}, J=7.6,1.8 \mathrm{~Hz}, 4 \mathrm{H}), 7.38-7.28$ (m, $6 \mathrm{H}), 6.98(\mathrm{dd}, J=7.7,1.3 \mathrm{~Hz}, 1 \mathrm{H}), 6.81(\operatorname{app~t}, J=7.9 \mathrm{~Hz}, 1 \mathrm{H}), 6.49(\mathrm{dd}, J=8.1,1.4 \mathrm{~Hz}, 1 \mathrm{H}), 4.33(\mathrm{t}, J=$ $6.2 \mathrm{~Hz}, 1 \mathrm{H}), 3.57(\mathrm{~d}, J=6.2 \mathrm{~Hz}, 2 \mathrm{H}), 2.76(\mathrm{~s}, 1 \mathrm{H}), 1.72(\mathrm{~s}, 3 \mathrm{H}), 1.61(\mathrm{~s}, 3 \mathrm{H}), 1.02(\mathrm{~s}, 9 \mathrm{H}) ;{ }^{13} \mathrm{C} \mathrm{NMR}$ 
$\left(\mathrm{CDCl}_{3}, 75 \mathrm{MHz}\right) \delta 165.0,148.7,142.8,135.3,134.0,129.0,127.2,123.7,120.8,110.3,104.5,53.3,46.7$, 28.8, 27.9, 26.8, 25.7, 20.0; HRMS(EI) $\mathrm{m} / z$ calcd for $\mathrm{C}_{30} \mathrm{H}_{34} \mathrm{O}_{6} \mathrm{Si}\left(\mathrm{M}^{+}-\mathrm{CH}_{3}\right)$ : 503.1890. Found: 503.1905.

4-[1-(tert-Butyl)-1,1-diphenylsilyl]oxy-5-methoxy-1-indanone (43): Prepared from 42 in $50 \%$ yield using Procedure D in $40 \mathrm{~min}$. Purified by flash chromatography (10:1 hexanes:EtOAc) to provide a pale yellow oil. ${ }^{1} \mathrm{H}$ NMR $\left(\mathrm{CDCl}_{3}, 300 \mathrm{MHz}\right) \delta 7.66(\mathrm{~d}, J=7.1 \mathrm{~Hz}, 4 \mathrm{H}), 7.37-7.29(\mathrm{~m}, 4 \mathrm{H}), 6.65(\mathrm{~d}, J=$ 8.3 Hz, 1H), 3.11-3.07 (m, 2H), 3.09 (s, 3H), 2.66-2.63 (AB m, 2H), 1.09 (s, 9H); ${ }^{13} \mathrm{C} \mathrm{NMR}\left(\mathrm{CDCl}_{3}, 75\right.$ MHz) $\delta 205.8,154.5,145.7,141.5,134.7,134.1,131.1,129.4,127.3,117.6,111.6,54.4,36.5,26.7,23.2$, 20.0; $\mathrm{HRMS}(\mathrm{EI}) \mathrm{m} / z$ calcd for $\mathrm{C}_{26} \mathrm{H}_{28} \mathrm{O}_{3} \mathrm{Si}\left(\mathrm{M}^{+}-\mathrm{CH}_{3}\right): 401.1573$. Found: 401.1581.

\section{5-3-Methoxy-2-[(1,1,1-triisopropylsilyl)oxy]benzyl-2,2-dimethyl-1,3-dioxane-4,6-dione (44):}

Reductive alkylation of Meldrum's acid with TIPS-protected $o$-vanillin according to Procedure A, followed by flash chromatography (6:1 hexanes:EtOAc) and recrystallization from $\mathrm{MeOH}$ gave a $21 \%$ yield of white solid. M.p. $77-78{ }^{\circ} \mathrm{C}(\mathrm{MeOH}) ;{ }^{1} \mathrm{H} \mathrm{NMR}\left(\mathrm{CDCl}_{3}, 300 \mathrm{MHz}\right) \delta 6.96(\mathrm{dd}, J=7.5,1.3 \mathrm{~Hz}, 1 \mathrm{H}), 6.83$ (app t, $J=7.8 \mathrm{~Hz}, 1 \mathrm{H}), 6.74(\mathrm{dd}, J=8.1,1.4 \mathrm{~Hz}, 1 \mathrm{H}), 4.15(\mathrm{t}, J=6.0 \mathrm{~Hz}, 1 \mathrm{H}), 3.79(\mathrm{~s}, 3 \mathrm{H}), 3.39(\mathrm{~d}, J=6.0 \mathrm{~Hz}$, 2H), 1.79 (s, 3H), $1.76(\mathrm{~s}, 3 \mathrm{H}), 1.28$ (septet, $J=7.7 \mathrm{~Hz}, 3 \mathrm{H}), 1.10(\mathrm{~d}, J=7.3 \mathrm{~Hz}, 18 \mathrm{H}) ;{ }^{13} \mathrm{C} \mathrm{NMR}\left(\mathrm{CDCl}_{3}\right.$, $75 \mathrm{MHz}) \delta 165.0,149.3,143.5,128.3,124.1,120.3,109.1,104.7,54.5,46.6,28.7,27.7,25.9,18.0,14.2$; HRMS(EI) $m / z$ calcd for $\mathrm{C}_{23} \mathrm{H}_{36} \mathrm{O}_{6} \mathrm{Si}\left(\mathrm{M}^{+}-\mathrm{CH}_{3}\right)$ : 421.2046. Found: 421.2056.

5-Methoxy-4-[(1,1,1-triisopropylsilyl)oxy]-1-indanone (45): Prepared from 44 in $45 \%$ yield using Procedure D in 40 min. Purified by flash chromatography (12:1 hexanes:EtOAc) to provide a pale yellow solid. M.p. 59-60 ${ }^{\circ} \mathrm{C}\left(\mathrm{Et}_{2} \mathrm{O}\right) ;{ }^{1} \mathrm{H} \mathrm{NMR}\left(\mathrm{CDCl}_{3}, 300 \mathrm{MHz}\right) \delta 7.36(\mathrm{~d}, J=8.3 \mathrm{~Hz}, 1 \mathrm{H}), 6.88(\mathrm{~d}, J=8.3 \mathrm{~Hz}$, 1H), $3.85(\mathrm{~s}, 3 \mathrm{H}), 3.04(\mathrm{t}, J=5.9 \mathrm{~Hz}, 2 \mathrm{H}), 2.64(\mathrm{dd}, J=6.1,4.8 \mathrm{~Hz}, 2 \mathrm{H}), 1.24$ (septet, $J=7.9 \mathrm{~Hz}, 3 \mathrm{H})$, $1.07(\mathrm{~d}, J=7.1 \mathrm{~Hz}, 18 \mathrm{H}) ;{ }^{13} \mathrm{C} \mathrm{NMR}\left(\mathrm{CDCl}_{3}, 75 \mathrm{MHz}\right) \delta 206.0,154.9,146.1,142.2,131.2,117.1,111.4$, 55.3, 36.5, 23.1, 18.0, 13.7; HRMS(EI) $m / z$ calcd for $\mathrm{C}_{19} \mathrm{H}_{30} \mathrm{O}_{3} \mathrm{Si}\left(\mathrm{M}^{+}\right)$: 334.1964. Found: 334.1966.

2,2-Dimethyl-5-(1-phenylcyclohexyl)-1,3-dioxane-4,6-dione (48): Conjugate addition of phenylmagnesium bromide to cyclohexyl Meldrum's alkylidene according to Procedure C provided a $66 \%$ yield of product following recrystallization from hexanes. M.p. 106-107 ${ }^{\circ} \mathrm{C}$ (hexanes); ${ }^{1} \mathrm{H} \mathrm{NMR}\left(\mathrm{CDCl}_{3}\right.$, $300 \mathrm{MHz}) \delta$ 7.35-7.22 (m, 5H), $3.44(\mathrm{~s}, 1 \mathrm{H}), 2.5-2.45(\mathrm{~m}, 2 \mathrm{H}), 2.05-1.97(\mathrm{~m}, 2 \mathrm{H}), 1.66-1.61(\mathrm{~m}, 2 \mathrm{H}), 1.45$ (s, 3H), 1.45-1.32 (m, 4H), $0.71(\mathrm{~s}, 3 \mathrm{H}) ;{ }^{13} \mathrm{C} \mathrm{NMR}\left(\mathrm{CDCl}_{3}, 75 \mathrm{MHz}\right) \delta 164.4,139.6,128.7,127.7,127.3$, 105.7, 57.2, 47.2, 35.5, 30.6, 26.2, 25.7, 22.3; Anal. Calcd for $\mathrm{C}_{18} \mathrm{H}_{22} \mathrm{O}_{4}: \mathrm{C}, 71.50 ; \mathrm{H}, 7.33$. Found: C, $71.40 ; \mathrm{H}, 7.32$. 
(3.3)-Pentamethylene-1-indanone ${ }^{12}$ (49): Prepared from 48 in 56\% yield using Procedure D in 30 min. Purified by flash chromatography using 7:1 hexanes:EtOAc to provide a colorless oil. Lit. M.p. 58-59 ${ }^{\circ} \mathrm{C}$ (petroleum ether); ${ }^{1} \mathrm{H} \mathrm{NMR}\left(\mathrm{CDCl}_{3}, 300 \mathrm{MHz}\right) \delta 7.65(\mathrm{~d}, J=7.7 \mathrm{~Hz}, 1 \mathrm{H}), 7.54(\mathrm{t}, J=7.1 \mathrm{~Hz}, 1 \mathrm{H})$, $7.45(\mathrm{~d}, J=7.7 \mathrm{~Hz}, 1 \mathrm{H}), 7.30(\mathrm{t}, J=7.4 \mathrm{~Hz}, 1 \mathrm{H}), 2.53(\mathrm{~s}, 3 \mathrm{H}), 1.75-1.20(\mathrm{~m}, 10 \mathrm{H}) ;{ }^{13} \mathrm{C} \mathrm{NMR}\left(\mathrm{CDCl}_{3}, 75\right.$ $\mathrm{MHz}) \delta 205.9,164.0,135.4,134.7,127.4,123.8,123.3,48.2,43.0,38.2,25.3,23.7$. Anal. calcd for $\mathrm{C}_{14} \mathrm{H}_{16} \mathrm{O}: \mathrm{C}, 83.96 ; \mathrm{H}, 8.05$. Found: C, 83.60; H, 8.12.

5-(5-Chloro-1-methyl-1-phenylpentyl)-2,2-dimethyl-1,3-dioxane-4,6-dione (50): Meldrum's acid and 6-chloro-2-hexanone were condensed according to the procedure of Brown ${ }^{13}$, to provide the chlorobutyl methyl Meldrum's alkylidene in quantitative yield and was used without further purification. Subsequent conjugate addition according to Procedure $\mathrm{C}$ using phenylmagnesium bromide produced the desired compound in 51\% yield as an oil following flash chromatography (5:1 hexanes:EtOAc). M.p. 84$85{ }^{\circ} \mathrm{C}(\mathrm{MeOH}) ;{ }^{1} \mathrm{H}$ NMR $\left(\mathrm{CDCl}_{3}, 300 \mathrm{MHz}\right) \delta$ 7.34-7.20 (m, 5H), $3.60(\mathrm{~s}, 1 \mathrm{H}), 3.46(\mathrm{t}, J=6.6 \mathrm{~Hz}, 2 \mathrm{H})$, 2.14-2.06 (m, 2H), 1.77-1.68 (m, 2H), $1.64(\mathrm{~s}, 3 \mathrm{H}), 1.59$ (s, 3H), 1.42-1.37 (m, 1H), 1.18 (s, 3H), 1.18$1.08(\mathrm{~m}, 1 \mathrm{H}) ;{ }^{13} \mathrm{C} \mathrm{NMR}\left(\mathrm{CDCl}_{3}, 75 \mathrm{MHz}\right) \delta 164.4,163.9,142.3,128.5,127.1,126.6,105.2,57.2,45.8$, 44.6, 39.3, 32.8, 29.3, 27.2, 22.3, 21.7; $\mathrm{HRMS}(\mathrm{EI}) \mathrm{m} / \mathrm{z}$ calcd for $\mathrm{C}_{18} \mathrm{H}_{23} \mathrm{ClO}_{4}\left(\mathrm{M}^{+}\right)$: 338.1285. Found: 338.1292.

3-(4-Chlorobutyl)-3-methyl-1-indanone (51): Prepared from 50 in 52\% yield using Procedure D in 15 min. Purified by flash chromatography (8:1 hexanes:EtOAc) to provide a pale yellow oil. ${ }^{1} \mathrm{H}$ NMR $\left(\mathrm{CDCl}_{3}, 300 \mathrm{MHz}\right) \delta 7.68(\mathrm{~d}, J=7.7 \mathrm{~Hz}, 1 \mathrm{H}), 7.59(\mathrm{t}, J=7.1 \mathrm{~Hz}, 1 \mathrm{H}), 7.43(\mathrm{~d}, J=7.7 \mathrm{~Hz}, 1 \mathrm{H}), 7.35(\mathrm{t}, J$ $=7.5 \mathrm{~Hz}, 1 \mathrm{H}), 3.43(\mathrm{t}, J=6.6 \mathrm{~Hz}, 2 \mathrm{H}), 2.54(\mathrm{AB}$ quartet, $J=18.9 \mathrm{~Hz}, 2 \mathrm{H}), 1.76-1.60(\mathrm{~m}, 4 \mathrm{H}), 1.50-1.39$ (m, 1H), $1.39(\mathrm{~s}, 3 \mathrm{H}), 1.1-0.95(\mathrm{~m}, 1 \mathrm{H}) ;{ }^{13} \mathrm{C} \mathrm{NMR}\left(\mathrm{CDCl}_{3}, 75 \mathrm{MHz}\right) \delta 205.8,162.4,135.9,134.9,127.5$, 123.7, 123.3, 50.0, 44.5, 41.9, 41.3, 32.9, 28.3, 22.3; HRMS(EI) $m / z$ calcd for $\mathrm{C}_{14} \mathrm{H}_{17} \mathrm{ClO}\left(\mathrm{M}^{+}\right): 236.0968$. Found: 236.0971.

5-(3-Methoxybenzyl)-2,2-dimethyl-1,3-dioxane-4,6-dione (52): Prepared by reductive alkylation of Meldrum's acid with $m$-anisaldehyde using Procedure A in $45 \%$ yield after recrystallization from $\mathrm{MeOH}$. M.p. $102-104{ }^{\circ} \mathrm{C}(\mathrm{MeOH}) ;{ }^{1} \mathrm{H}$ NMR $\left(\mathrm{CDCl}_{3}, 300 \mathrm{MHz}\right) \delta 7.15($ app t, $J=8.0 \mathrm{~Hz}, 1 \mathrm{H}), 6.86-6.84$ (m, 2H), 6.75-6.72 (m, 1H), $3.77(\mathrm{t}, J=5.0 \mathrm{~Hz}, 1 \mathrm{H}), 3.73(\mathrm{~s}, 3 \mathrm{H}), 3.40(\mathrm{~d}, J=5.0 \mathrm{~Hz}, 2 \mathrm{H}), 1.69(\mathrm{~s}, 3 \mathrm{H}), 1.49$ $(\mathrm{s}, 3 \mathrm{H}) ;{ }^{13} \mathrm{C} \mathrm{NMR}\left(\mathrm{CDCl}_{3}, 75 \mathrm{MHz}\right) \delta 165.2,159.4,138.7,129.4,121.7,115.1,112.5,105.1,55.0,47.8$, 31.8, 28.2, 26.8; Anal. Calcd for $\mathrm{C}_{14} \mathrm{H}_{16} \mathrm{O}_{5}$ : C, 63.63; H, 6.10. Found: C, 63.70; H, 6.15. 
5-Methoxy-1-indanone (53) and 7-Methoxy-1-indanone (54): Prepared from 52 in $52 \%$ yield as a 5.5:1 ratio of para 53:ortho 54 products using Procedure D in $60 \mathrm{~min}$. The mixture was separated with 2:1 hexanes:EtOAc; (53): M.p. 108-109 ${ }^{\circ} \mathrm{C}(\mathrm{MeOH}) ;{ }^{1} \mathrm{H}$ NMR $\left(\mathrm{CDCl}_{3}, 300 \mathrm{MHz}\right) \delta$ 7.68-7.65 (m, 1H), 6.89$6.87(\mathrm{~m}, 2 \mathrm{H}), 3.86(\mathrm{~s}, 3 \mathrm{H}), 3.07(\mathrm{~m}, 2 \mathrm{H}), 2.65(\mathrm{~m}, 2 \mathrm{H}) ;{ }^{13} \mathrm{C} \mathrm{NMR}\left(\mathrm{CDCl}_{3}, 75 \mathrm{MHz}\right) \delta 205.3,165.2,158.2$, 130.4, 125.3, 115.3, 109.7, 55.6, 36.4, 25.8. Anal. calcd for $\mathrm{C}_{10} \mathrm{H}_{10} \mathrm{O}_{2}: \mathrm{C}, 74.06 ; \mathrm{H}, 6.21$. Found: C, 73.90; H, 6.27. (54): M.p. 96-97.5 ${ }^{\circ} \mathrm{C} ;{ }^{1} \mathrm{H}$ NMR $\left(\mathrm{CDCl}_{3}, 300 \mathrm{MHz}\right) \delta 7.50(\mathrm{t}, J=7.9 \mathrm{~Hz}, 1 \mathrm{H}), 6.99(\mathrm{~d}, J=7.5$ $\mathrm{Hz}, 1 \mathrm{H}), 6.76(\mathrm{~d}, J=8.0 \mathrm{~Hz}, 1 \mathrm{H}), 3.93(\mathrm{~s}, 3 \mathrm{H}), 3.08-3.04(\mathrm{~m}, 2 \mathrm{H}), 2.68-2.63(\mathrm{~m}, 2 \mathrm{H}) ;{ }^{13} \mathrm{C} \mathrm{NMR}\left(\mathrm{CDCl}_{3}\right.$, $75 \mathrm{MHz}) \delta 204.9,158.1,158.0,136.4,125.2,118.4,108.8,55.7,36.8,25.5$. HRMS(EI): $m / z$ calcd for $\mathrm{C}_{10} \mathrm{H}_{10} \mathrm{O}_{2}\left(\mathrm{M}^{+}\right):$162.0681. Found 162.0685.

5-[1-(3-Methoxyphenyl)cyclohexyl]-2,2-dimethyl-1,3-dioxane-4,6-dione (55): Conjugate addition of 3-methoxyphenylmagnesium bromide to cyclohexyl Meldrum's alkylidene according to Procedure C provided a solid in 36\% yield after flash chromatography (4:1 hexanes:EtOAc) and recrystallization from MeOH. M.p. 88-89 ${ }^{\circ} \mathrm{C}(\mathrm{MeOH}) ;{ }^{1} \mathrm{H}$ NMR $\left(\mathrm{CDCl}_{3}, 300 \mathrm{MHz}\right)$ 8 7.27-7.21 (m, 2H), 6.89-6.76 (m, 3H), $3.76(\mathrm{~s}, 3 \mathrm{H}), 3.43(\mathrm{~s}, 1 \mathrm{H}), 2.55-2.40(\mathrm{~m}, 2 \mathrm{H}), 2.10-1.90(\mathrm{~m}, 2 \mathrm{H}), 1.70-1.50(\mathrm{~m}, 2 \mathrm{H}), 1.52-1.30(\mathrm{~m}, 4 \mathrm{H})$, $1.47(\mathrm{~s}, 3 \mathrm{H}), 0.81(\mathrm{~s}, 3 \mathrm{H}) ;{ }^{13} \mathrm{C} \mathrm{NMR}\left(\mathrm{CDCl}_{3}, 75 \mathrm{MHz}\right) \delta 164.4,159.9,141.4,129.6,120.0,113.9,112.5$, 105.6, 57.0, 55.2, 47.2, 35.6, 30.5, 26.4, 25.6, 22.3; Anal. Calcd for $\mathrm{C}_{19} \mathrm{H}_{24} \mathrm{O}_{5}: \mathrm{C}, 68.66 ; \mathrm{H}, 7.28$. Found: C, 68.64; H, 7.39.

\section{5-Methoxy-(3.3)-pentamethylene-1-indanone (56) and 7-Methoxy-(3.3)-pentamethylene-1-}

indanone $^{14}$ (57): Prepared from 55 in $71 \%$ yield as a 3.4:1 ratio of para 56:ortho 57 products using Procedure D in $120 \mathrm{~min}$. The mixture was separated with 3:1 hexanes:EtOAc; (56): M.p. 82-82.5 ${ }^{\circ} \mathrm{C}$ (hexanes). Lit. 83.5-85 ${ }^{\circ} \mathrm{C} ;{ }^{1} \mathrm{H}$ NMR $\left(\mathrm{CDCl}_{3}, 300 \mathrm{MHz}\right) \delta 7.60$ (d, J=9.0 Hz, 1H), 6.87-6.84 (m, 2H), $3.86(\mathrm{~s}, 3 \mathrm{H}), 2.53(\mathrm{~s}, 2 \mathrm{H}), 1.77-1.26(\mathrm{~m}, 10 \mathrm{H}) ;{ }^{13} \mathrm{C} \mathrm{NMR}\left(\mathrm{CDCl}_{3}, 75 \mathrm{MHz}\right) \delta 204.2,167.1,165.3,128.8$ 125.1, 115.1, 107.4, 55.6, 48.5, 42.9, 38.2, 25.4, 23.7. Anal. calcd for $\mathrm{C}_{15} \mathrm{H}_{18} \mathrm{O}_{2}$ : C, 78.23; H, 7.88. Found: C, 78.38; H, 7.84. (57): M.p. $94-96{ }^{\circ} \mathrm{C}$. Lit. $97.5-99{ }^{\circ} \mathrm{C}\left(\mathrm{Et}_{2} \mathrm{O}\right) ;{ }^{1} \mathrm{H} \mathrm{NMR}\left(\mathrm{CDCl}_{3}, 300 \mathrm{MHz}\right) \delta 7.52(\mathrm{t}, J=$ $7.9 \mathrm{~Hz}, 1 \mathrm{H}), 7.02(\mathrm{~d}, J=7.7 \mathrm{~Hz}, 1 \mathrm{H}), 6.55(\mathrm{~d}, J=8.2 \mathrm{~Hz}, 1 \mathrm{H}), 3.92(\mathrm{~s}, 3 \mathrm{H}), 2.55(\mathrm{~s}, 2 \mathrm{H}), 1.77-1.25$ (m, $10 \mathrm{H}) ;{ }^{13} \mathrm{C} \mathrm{NMR}\left(\mathrm{CDCl}_{3}, 75 \mathrm{MHz}\right) \delta 203.9,167.0,157.6,136.5,123.6,115.6,108.8,55.8,48.9,42.5,38.3$, 25.5, 23.7. $\mathrm{HRMS}(\mathrm{EI}): \mathrm{m} / z$ calcd for $\mathrm{C}_{15} \mathrm{H}_{18} \mathrm{O}_{2}\left(\mathrm{M}^{+}\right)$: 230.1307. Found: 230.1304.

2,2-Dimethyl-5-(3-methylbenzyl)-1,3-dioxane-4,6-dione (58): Reductive alkylation of Meldrum's acid with $m$-tolualdehyde according to Procedure A provided a white solid in $69 \%$ yield following SI-1-14 
recrystallization from $\mathrm{MeOH}$. M.p. $81-82.5{ }^{\circ} \mathrm{C}(\mathrm{MeOH}) ;{ }^{1} \mathrm{H}$ NMR $\left(\mathrm{CDCl}_{3}, 300 \mathrm{MHz}\right) \delta$ 7.15-7.01 (m, $4 \mathrm{H}), 3.76(\mathrm{t}, J=4.9 \mathrm{~Hz}, 1 \mathrm{H}), 3.41(\mathrm{~d}, J=4.9 \mathrm{~Hz}, 2 \mathrm{H}), 2.30(\mathrm{~s}, 3 \mathrm{H}), 1.71(\mathrm{~s}, 3 \mathrm{H}), 1.48(\mathrm{~s}, 3 \mathrm{H}) ;{ }^{13} \mathrm{C} \mathrm{NMR}$ $\left(\mathrm{CDCl}_{3}, 75 \mathrm{MHz}\right) \delta 165.3,138.1,137.1,130.3,128.3,127.7,126.5,105.1,47.9,31.8,38.3,27.0,21.2$; Anal. calcd for $\mathrm{C}_{14} \mathrm{H}_{16} \mathrm{O}_{4}$ : C, 67.73; H, 6.50. Found: C, 67.67; H, 6.46.

5-Methyl-1-indanone (59) and 7-Methyl-1-indanone ${ }^{15}(60)$ : Prepared from 58 in $48 \%$ yield as a 1:1 ratio of para 59:ortho 60 products using Procedure D in $60 \mathrm{~min}$. The mixture was separated with 10:1 hexanes:EtOAc; (59): M.p 67-68 ${ }^{\circ} \mathrm{C}$ (petroleum ether). Lit. 68-69 ${ }^{\circ} \mathrm{C} ;{ }^{1} \mathrm{H}$ NMR $\left(\mathrm{CDCl}_{3}, 300 \mathrm{MHz}\right) \delta 7.62$ (d, $J=7.8 \mathrm{~Hz}, 1 \mathrm{H}), 7.25$ (br s, 1H), $7.15(\mathrm{~d}, J=7.9 \mathrm{~Hz}, 1 \mathrm{H}), 3.08-3.04(\mathrm{~m}, 2 \mathrm{H}), 2.67-2.63$ (m, $2 \mathrm{H}), 2.41$ $(\mathrm{s}, 3 \mathrm{H}) ;{ }^{13} \mathrm{C} \mathrm{NMR}\left(\mathrm{CDCl}_{3}, 75 \mathrm{MHz}\right) \delta 206.6,155.7,145.7,134.8,128.5,127.0,123.5,36.4,25.6,22.0$. HRMS(EI): $m / z$ calcd for $\mathrm{C}_{10} \mathrm{H}_{10} \mathrm{O}\left(\mathrm{M}^{+}\right)$: 146.0732. Found: 146.0732. (60): M.p. 49-50 ${ }^{\circ} \mathrm{C}$ (petroleum ether). Lit. 52.5-53.5 ${ }^{\circ} \mathrm{C} ;{ }^{1} \mathrm{H} \mathrm{NMR}\left(\mathrm{CDCl}_{3}, 300 \mathrm{MHz}\right) \delta 7.38(\mathrm{t}, J=7.5 \mathrm{~Hz}, 1 \mathrm{H}), 7.23(\mathrm{~d}, J=7.4 \mathrm{~Hz}, 1 \mathrm{H})$, $7.04(\mathrm{~d}, J=7.4 \mathrm{~Hz}, 1 \mathrm{H}), 3.05-3.01(\mathrm{~m}, 2 \mathrm{H}), 2.62-2.58(\mathrm{~m}, 2 \mathrm{H}), 2.59(\mathrm{~s}, 3 \mathrm{H}) ;{ }^{13} \mathrm{C} \mathrm{NMR}\left(\mathrm{CDCl}_{3}, 75 \mathrm{MHz}\right)$ $\delta 207.8,155.8,138.6,134.3,133.8,128.9,123.9,36.6,25.2,18.2$. HRMS(EI): $\mathrm{m} / z$ calcd for $\mathrm{C}_{10} \mathrm{H}_{10} \mathrm{O}$ $\left(\mathrm{M}^{+}\right)$: 146.0732. Found: 146.0728 .

2,2-Dimethyl-5-[1-(3-methylphenyl)cyclohexyl]-1,3-dioxane-4,6-dione (61): Prepared by conjugate addition of 3-methylphenylmagnesium bromide to cyclohexyl Meldrum's alkylidene according to Procedure C. A white solid was obtained in $35 \%$ yield after flash chromatography $(5: 1$ hexanes:EtOAc) and recrystallization from MeOH. M.p. $124-125{ }^{\circ} \mathrm{C}(\mathrm{MeOH}) ;{ }^{1} \mathrm{H}$ NMR $\left(\mathrm{CDCl}_{3}, 300\right.$ $\mathrm{MHz}) \delta 7.21-6.90(\mathrm{~m}, 4 \mathrm{H}), 3.42(\mathrm{~s}, 1 \mathrm{H}), 2.50-2.40(\mathrm{~m}, 2 \mathrm{H}), 2.28(\mathrm{~s}, 3 \mathrm{H}), 2.00-1.90(\mathrm{~m}, 2 \mathrm{H}), 1.75-1.55$ (m, 2H), $1.44(\mathrm{~s}, 3 \mathrm{H}), 1.44-1.30(\mathrm{~m}, 4 \mathrm{H}), 0.69(\mathrm{~s}, 3 \mathrm{H}) ;{ }^{13} \mathrm{C} \mathrm{NMR}\left(\mathrm{CDCl}_{3}, 75 \mathrm{MHz}\right) \delta 164.3,139.6,138.1$, $128.5,128.1,127.9,124.6,105.5,56.9,47.0,35.5,30.5,26.1,25.6,22.2,21.5$; Anal. Calcd for $\mathrm{C}_{19} \mathrm{H}_{24} \mathrm{O}_{4}$ : C, 72.13; H, 7.65. Found: C, 72.09; H, 7.63.

\section{5-Methyl-(3.3)-pentamethylene-1-indanone (62) and 7-Methyl-(3.3)-pentamethylene-1-}

indanone (63): Prepared from 61 in $62 \%$ yield as a 1:1 ratio of para 62:ortho 63 products using Procedure D in 120 min. The resulting mixture of $\mathbf{6 2}$ and 63 was separated with 10:1 hexanes:EtOAc; (62): M.p. $77.5-78.5^{\circ} \mathrm{C} ;{ }^{1} \mathrm{H}$ NMR $\left(\mathrm{CDCl}_{3}, 300 \mathrm{MHz}\right) \delta 7.58(\mathrm{~d}, J=7.8 \mathrm{~Hz}, 1 \mathrm{H}), 7.27$ (s, $\left.1 \mathrm{H}\right), 7.15(\mathrm{~d}, J$ $=7.8 \mathrm{~Hz}, 1 \mathrm{H}), 2.55(\mathrm{~s}, 2 \mathrm{H}), 2.43(\mathrm{~s}, 3 \mathrm{H}), 1.79-1.27(\mathrm{~m}, 10 \mathrm{H}) ;{ }^{13} \mathrm{C} \mathrm{NMR}\left(\mathrm{CDCl}_{3}, 75 \mathrm{MHz}\right) \delta 205.6,164.7$, 145.9, 133.3, 128.8, 124.3, 123.2, 48.5, 42.9, 38.3, 25.5, 23.8, 22.2. HRMS(EI): $\mathrm{m} / z$ calcd for $\mathrm{C}_{15} \mathrm{H}_{18} \mathrm{O}$ $\left(\mathrm{M}^{+}\right)$214.1358. Found 214.1354. (63): Oil; ${ }^{1} \mathrm{H}$ NMR $\left(\mathrm{CDCl}_{3}, 300 \mathrm{MHz}\right) \delta 7.42(\mathrm{t}, J=7.5 \mathrm{~Hz}, 1 \mathrm{H}), 7.29$ 
$(\mathrm{d}, J=7.7 \mathrm{~Hz}, 1 \mathrm{H}), 7.07(\mathrm{~d}, J=7.3 \mathrm{~Hz}, 1 \mathrm{H}), 2.61(\mathrm{~s}, 3 \mathrm{H}), 2.53(\mathrm{~s}, 2 \mathrm{H}), 1.78-1.23(\mathrm{~m}, 10 \mathrm{H}) ;{ }^{13} \mathrm{C}$ NMR $\left(\mathrm{CDCl}_{3}, 75 \mathrm{MHz}\right) \delta 207.1,165.1$ 138.5, 134.0, 133.0, 129.3, 121.2, 48.9, 42.3, 38.5, 25.5, 23.8, 18.4. Anal. calcd for $\mathrm{C}_{15} \mathrm{H}_{18} \mathrm{O}: \mathrm{C}, 84.07 ; \mathrm{H}, 8.47$. Found: C, 84.19; H, 8.36.

5-(3-Chlorobenzyl)-2,2-dimethyl-1,3-dioxane-4,6-dione (64): Reductive alkylation of Meldrum's acid with 3-chlorobenzaldehyde according to Procedure A produced white needles in $51 \%$ yield following recrystallization from $\mathrm{MeOH}$. M.p. $105-105{ }^{\circ} \mathrm{C}(\mathrm{MeOH}) ;{ }^{1} \mathrm{H} \mathrm{NMR}\left(\mathrm{CDCl}_{3}, 300 \mathrm{MHz}\right) \delta 7.31(\mathrm{~s}, 1 \mathrm{H})$, $7.19(\mathrm{~s}, 3 \mathrm{H}), 3.74(\mathrm{t}, J=5.0 \mathrm{~Hz}, 1 \mathrm{H}), 3.41(\mathrm{~d}, J=5.0 \mathrm{~Hz}, 2 \mathrm{H}), 1.73(\mathrm{~s}, 3 \mathrm{H}), 1.58(\mathrm{~s}, 3 \mathrm{H}) ;{ }^{13} \mathrm{C} \mathrm{NMR}$ $\left(\mathrm{CDCl}_{3}, 75 \mathrm{MHz}\right) \delta 164.9,139.2,134.2,129.8,128.0,127.3,105.2,47.9,31.3,28.4,26.9 . \mathrm{HRMS}(\mathrm{EI}): \mathrm{m} / z$ calcd for $\mathrm{C}_{13} \mathrm{H}_{13} \mathrm{ClO}_{4}\left(\mathrm{M}^{+}\right)$: 268.0502. Found 268.0493.

5-[1-(3-Chlorophenyl)cyclohexyl]-2,2-dimethyl-1,3-dioxane-4,6-dione (65): Conjugate addition of 3-chlorophenylmagnesium bromide to cyclohexyl Meldrum's alkylidene according to Procedure C provided a 51\% yield of a white solid following flash chromatography (4:1 hexanes:EtOAc) and recrystallization from $\mathrm{MeOH}$. M.p. $115-116{ }^{\circ} \mathrm{C}(\mathrm{MeOH}) ;{ }^{1} \mathrm{H}$ NMR $\left(\mathrm{CDCl}_{3}, 300 \mathrm{MHz}\right) \delta$ 7.29-7.14 (m, $4 \mathrm{H}), 3.44(\mathrm{~s}, 1 \mathrm{H}), 2.50-2.30(\mathrm{~m}, 2 \mathrm{H}), 2.10-1.90(\mathrm{~m}, 2 \mathrm{H}), 1.75-1.65(\mathrm{~m}, 2 \mathrm{H}), 1.48(\mathrm{~s}, 3 \mathrm{H}), 1.48-1.30(\mathrm{~m}$, 4H), $0.88(\mathrm{~s}, 3 \mathrm{H}) ;{ }^{13} \mathrm{C} \mathrm{NMR}\left(\mathrm{CDCl}_{3}, 75 \mathrm{MHz}\right) \delta 164.2,142.1,134.7,129.9,128.0,127.5,126.0,105.6$, 56.9, 47.0, 35.4, 30.4, 26.4, 25.5, 22.2; Anal. Calcd for $\mathrm{C}_{18} \mathrm{H}_{21} \mathrm{ClO}_{4}$ : C, 64.19; H, 6.28. Found: C, 64.16; $\mathrm{H}$, 6.39 .

\section{5-Chloro-(3.3)-pentamethylene-1-indanone (66) and 7-Chloro-(3.3)-pentamethylene-1-} indanone (67): Prepared from 65 in 62\% yield as a 2:1 ratio of para 66:ortho 67 products using Procedure D in 120 min. The mixture was separated with $3: 1 \mathrm{CH}_{2} \mathrm{Cl}_{2}$ :hexanes; (66): M.p. $74-75{ }^{\circ} \mathrm{C} ;{ }^{1} \mathrm{H}$ NMR $\left(\mathrm{CDCl}_{3}, 300 \mathrm{MHz}\right) \delta 7.61(\mathrm{~d}, J=8.2 \mathrm{~Hz}, 1 \mathrm{H}), 7.46(\mathrm{~d}, J=1.6 \mathrm{~Hz}, 1 \mathrm{H}), 7.30(\mathrm{dd}, J=8.1,1.6 \mathrm{~Hz}$, 1H), $2.57(3 \mathrm{H}, \mathrm{s}), 1.80-1.22(\mathrm{~m}, 10 \mathrm{H}) ;{ }^{13} \mathrm{C} \mathrm{NMR}\left(\mathrm{CDCl}_{3}, 75 \mathrm{MHz}\right) \delta 204.5,165.6,141.2,134.0,128.3$, 124.7, 124.4, 48.3, 43.2, 38.2, 25.3, 23.7. HRMS(EI): $\mathrm{m} / \mathrm{z}$ calcd for $\mathrm{C}_{14} \mathrm{H}_{15} \mathrm{ClO}\left(\mathrm{M}^{+}\right)$234.0811. Found 234.0807. (67): M.p. $159-160{ }^{\circ} \mathrm{C}$ (hexanes); ${ }^{1} \mathrm{H} \mathrm{NMR}\left(\mathrm{CDCl}_{3}, 300 \mathrm{MHz}\right) \delta 7.48(\mathrm{t}, J=7.7 \mathrm{~Hz}, 1 \mathrm{H}), 7.38$ $(\mathrm{d}, J=7.5 \mathrm{~Hz}, 1 \mathrm{H}), 7.27(\mathrm{~d}, J=7.6 \mathrm{~Hz}, 1 \mathrm{H}), 2.60(\mathrm{~s}, 2 \mathrm{H}), 1.80-1.24(\mathrm{~m}, 10 \mathrm{H}) ;{ }^{13} \mathrm{C} \mathrm{NMR}\left(\mathrm{CDCl}_{3}, 75\right.$

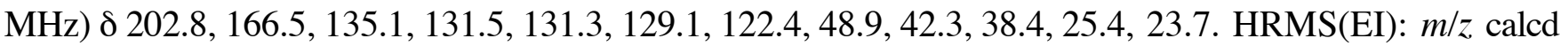
for $\mathrm{C}_{14} \mathrm{H}_{15} \mathrm{ClO}\left(\mathrm{M}^{+}\right): 234.0811$. Found 234.0806.

5-(3,5-Dimethoxybenzyl)-2,2,5-trimethyl-1,3-dioxane-4,6-dione (68): Prepared by alkylation of 9 according to Procedure B using iodomethane. Recrystallization from $i$-PrOH provided a white solid in 
79\% yield. M.p. $113-114{ }^{\circ} \mathrm{C}(i-\mathrm{PrOH}) ;{ }^{1} \mathrm{H} \mathrm{NMR}\left(\mathrm{CDCl}_{3}, 300 \mathrm{MHz}\right) \delta 6.24(\mathrm{~s}, 3 \mathrm{H}), 3.64(\mathrm{~s}, 6 \mathrm{H}), 3.18$ (s, 2H), $1.65(\mathrm{~s}, 3 \mathrm{H}), 1.53(\mathrm{~s}, 3 \mathrm{H}), 0.94(\mathrm{~s}, 3 \mathrm{H}) ;{ }^{13} \mathrm{C} \mathrm{NMR}\left(\mathrm{CDCl}_{3}, 75 \mathrm{MHz}\right) \delta 169.6,160.7,137.3,107.6$, 105.1, 99.9, 55.1, 51.9, 44.7, 29.1, 28.3, 26.1; Anal. Calcd for $\mathrm{C}_{16} \mathrm{H}_{20} \mathrm{O}_{6}: \mathrm{C}, 62.33$; H, 6.54. Found: C, $62.29 ; \mathrm{H}, 6.58$.

5,7-Dimethoxy-2-methyl-1-indanone ${ }^{16}$ (69): Prepared from 68 in $80 \%$ yield using Procedure D in 45 min. Purified with 1:1 hexanes:EtOAc; M.p. 71.5-73 ${ }^{\circ} \mathrm{C}$ (hexanes). Lit. 76-79 ${ }^{\circ} \mathrm{C}$; ${ }^{1} \mathrm{H}$ NMR $\left(\mathrm{CDCl}_{3}\right.$, $300 \mathrm{MHz}) \delta 6.44$ (br s, 1H), 6.28 (br s, 1H), 3.88 (s, 3H), 3.85 (s, 3H), 3.21-3.29 (m, 1H), 2.69-2.55 (m, $2 \mathrm{H}), 1.25(\mathrm{~d}, J=7.3 \mathrm{~Hz}, 3 \mathrm{H}) ;{ }^{13} \mathrm{C} \mathrm{NMR}\left(\mathrm{CDCl}_{3}, 75 \mathrm{MHz}\right) \delta 205.6,166.9,159.5,158.6,118.5,101.5$, 97.4, 55.7, 42.3, 35.0, 17.1. Anal. Calcd. for $\mathrm{C}_{12} \mathrm{H}_{14} \mathrm{O}_{3}:$ C, 69.88; H, 6.84. Found: C, 69.58; H, 6.71.

5-(3,5-Dimethylbenzyl)-2,2,5-trimethyl-1,3-dioxane-4,6-dione (70): Alkylation of 23 according to Procedure B using iodomethane, followed by recrystallization from $\mathrm{MeOH}$ gave a 38\% yield of white crystals. M.p. $117-118{ }^{\circ} \mathrm{C}(\mathrm{MeOH}) ;{ }^{1} \mathrm{H}$ NMR $\left(\mathrm{CDCl}_{3}, 300 \mathrm{MHz}\right) \delta 6.83(\mathrm{~s}, 1 \mathrm{H}), 6.75$ (s, $\left.2 \mathrm{H}\right), 3.22$ (s, 2H), $2.21(\mathrm{~s}, 6 \mathrm{H}), 1.71(\mathrm{~s}, 3 \mathrm{H}), 1.57(\mathrm{~s}, 3 \mathrm{H}), 0.88(\mathrm{~s}, 3 \mathrm{H}) ;{ }^{13} \mathrm{C} \mathrm{NMR}\left(\mathrm{CDCl}_{3}, 75 \mathrm{MHz}\right) \delta 169.8,138.2$, 135.1, 129.2, 127.7, 105.3, 52.1, 44.7, 29.3, 28.2, 26.0, 21.1; HRMS(EI) $m / z$ calcd for $\mathrm{C}_{16} \mathrm{H}_{20} \mathrm{O}_{4}\left(\mathrm{M}^{+}\right)$: 276.1361. Found: 276.1365 .

2,5,7-Trimethyl-1-indanone ${ }^{17}$ (71): Prepared from 70 in $87 \%$ yield using Procedure D in 30 min. Purified by flash chromatography (10:1 hexanes:EtOAc) to provide a clear colorless oil. ${ }^{1} \mathrm{H}$ NMR $\left(\mathrm{CDCl}_{3}\right.$, $300 \mathrm{MHz}) \delta 7.24(\mathrm{~s}, 1 \mathrm{H}), 6.85(\mathrm{~s}, 1 \mathrm{H}), 3.23(\mathrm{dd}, J=18.3,9.3 \mathrm{~Hz}, 1 \mathrm{H}), 2.62-2.53(\mathrm{~m}, 5 \mathrm{H}), 2.33(\mathrm{~s}, 3 \mathrm{H})$ $1.24(\mathrm{~d}, J=7.3 \mathrm{~Hz}, 3 \mathrm{H}) ;{ }^{13} \mathrm{C} \mathrm{NMR}\left(\mathrm{CDCl}_{3}, 75 \mathrm{MHz}\right) \delta 209.6,154.5,144.9,138.5,131.3,130.2,124.1$, 42.2, 34.2, 21.7, 18.0, 16.4; $\mathrm{HRMS}(\mathrm{EI}) \mathrm{m} / \mathrm{z}$ calcd for $\mathrm{C}_{12} \mathrm{H}_{14} \mathrm{O}\left(\mathrm{M}^{+}\right)$: 174.1045. Found: 174.1040.

5-(3,4-Dimethoxybenzyl)-2,2,5-trimethyl-1,3-dioxane-4,6-dione (72): Prepared by alkylation of 27 according to Procedure B using iodomethane. Recrystallization from $\mathrm{MeOH}$ provided a white solid in 76\% yield. M.p. $88-89{ }^{\circ} \mathrm{C}(\mathrm{MeOH}) ;{ }^{1} \mathrm{H} \mathrm{NMR}\left(\mathrm{CDCl}_{3}, 300 \mathrm{MHz}\right) \delta$ 6.68-6.60 (m, 3H), 3.74 (s, $\left.3 \mathrm{H}\right), 3.73$ (s, 3H), $3.18(\mathrm{~s}, 2 \mathrm{H}), 1.64(\mathrm{~s}, 3 \mathrm{H}), 1.52(\mathrm{~s}, 3 \mathrm{H}), 0.90(\mathrm{~s}, 3 \mathrm{H}) ;{ }^{13} \mathrm{C} \mathrm{NMR}\left(\mathrm{CDCl}_{3}, 75 \mathrm{MHz}\right) \delta 169.8,148.7$, 148.3, 127.6, 122.1, 112.8, 110.9, 105.0, 55.6, 52.2, 44.3, 29.1, 28.3, 25.7; Anal. Calcd for $\mathrm{C}_{16} \mathrm{H}_{20} \mathrm{O}_{6}: \mathrm{C}$, 62.33; H, 6.54. Found: C, 62.68; H, 6.68.

5,6-Dimethoxy-2-methyl-1-indanone ${ }^{18}$ (73): Prepared from 72 in $77 \%$ yield using Procedure D in 45 min. Purified by flash chromatography (2:1 hexanes:EtOAc) to provide a white powder. M.p. 131-132 ${ }^{\circ} \mathrm{C}\left(\mathrm{CH}_{2} \mathrm{Cl}_{2}\right)$. Lit. $132-133{ }^{\circ} \mathrm{C} ;{ }^{1} \mathrm{H}$ NMR $\left(\mathrm{CDCl}_{3}, 300 \mathrm{MHz}\right) \delta 7.08(\mathrm{~s}, 1 \mathrm{H}), 6.79(\mathrm{~s}, 1 \mathrm{H}), 3.88(\mathrm{~s}, 1 \mathrm{H}), 3.82$ 
$(\mathrm{s}, 1 \mathrm{H}), 3.22(\mathrm{dd}, J=16.4,7.2 \mathrm{~Hz}, 1 \mathrm{H}), 2.64-2.51(\mathrm{~m}, 2 \mathrm{H}), 1.20(\mathrm{~d}, J=7.2 \mathrm{~Hz}, 1 \mathrm{H}) ;{ }^{13} \mathrm{C} \mathrm{NMR}\left(\mathrm{CDCl}_{3}, 75\right.$ MHz) $\delta 207.9,155.3,149.3,148.5,128.8,107.3,104.3,56.0,55.9,42.0,34.6,16.5 ; \mathrm{HRMS} \mathrm{m} / z$ calcd for $\mathrm{C}_{12} \mathrm{H}_{14} \mathrm{O}_{3}\left(\mathrm{M}^{+}\right):$206.0943. Found: 206.0941 .

5-(3,4-Dimethoxybenzyl)-2,2-dimethyl-5-phenyl-1,3-dioxane-4,6-dione (74): Phenyl Meldrum's acid $^{19}$ was alkylated with 3,4-dimethoxybenzyl bromide ${ }^{20}$ according to Procedure B and purified by recrystallization from $\mathrm{MeOH}$ to provide a white solid in $68 \%$ yield. M.p. $99-100{ }^{\circ} \mathrm{C}(\mathrm{MeOH}) ;{ }^{1} \mathrm{H}$ NMR $\left(\mathrm{CDCl}_{3}, 300 \mathrm{MHz}\right) \delta$ 7.59-7.56 (m, 2H), 7.40-7.36 (m, 3H), 6.88-6.85 (m, 2H), 6.76-6.74 (m, 1H), $3.83(\mathrm{~s}$, 3H), $3.82(\mathrm{~s}, 3 \mathrm{H}), 3.61(\mathrm{~s}, 2 \mathrm{H}), 1.40(\mathrm{~s}, 3 \mathrm{H}), 1.31(\mathrm{~s}, 3 \mathrm{H}) ;{ }^{13} \mathrm{C} \mathrm{NMR}\left(\mathrm{CDCl}_{3}, 75 \mathrm{MHz}\right) \delta 167.0,148.5$, $148.3,136.1,129.4,128.8,127.6,126.5,123.3,114.0,110.9,105.4,61.9,55.8,55.7,45.1,28.9,28.4$; HRMS(EI) $m / z$ calcd for $\mathrm{C}_{21} \mathrm{H}_{22} \mathrm{O}_{6}\left(\mathrm{M}^{+}\right): 370.1416$. Found: 370.1411 .

5,6-Dimethoxy-2-phenyl-1-indanone ${ }^{21}$ (75): Prepared from 74 in $67 \%$ yield using Procedure D in 60 min. Purified by flash chromatography (4:1 hexanes:EtOAc) to provide a white solid. M.p. $155-157{ }^{\circ} \mathrm{C}$ (MeOH). Lit. 153-156 ${ }^{\circ} \mathrm{C} ;{ }^{1} \mathrm{H}$ NMR $\left(\mathrm{CDCl}_{3}, 300 \mathrm{MHz}\right) \delta$ 7.31-7.13 (m, 6H), 6.92 (s, 1H), 3.97 (s, 3H), $3.89(\mathrm{~s}, 3 \mathrm{H}), 3.84(\mathrm{dd}, J=8.0,3.5 \mathrm{~Hz}), 3.57(\mathrm{dd}, 17.2,8.0 \mathrm{~Hz}, 1 \mathrm{H}), 3.13(\mathrm{dd}, J=17.2,3.5 \mathrm{~Hz}, 1 \mathrm{H}) .{ }^{13} \mathrm{C}$ $\operatorname{NMR}\left(\mathrm{CDCl}_{3}, 75 \mathrm{MHz}\right) \delta 204.6,155.7,149.6,149.9,140.2,128.9,128.7,127.7,126.8,107.2,104.7,56.2$, 56.1, 53.6, 35.7; HRMS(EI) $\mathrm{m} / z$ calcd for $\mathrm{C}_{17} \mathrm{H}_{16} \mathrm{O}_{3}\left(\mathrm{M}^{+}\right)$: 268.1099. Found: 268.1107.

5-Benzyl-5-(3,4-dimethoxybenzyl)-2,2-dimethyl-1,3-dioxane-4,6-dione (76): Prepared by the alkylation of 27 according to Procedure B using benzyl bromide. Recrystallization from EtOH provided a clear colorless solid in $70 \%$ yield. M.p. $148-149{ }^{\circ} \mathrm{C}(\mathrm{EtOH}) ;{ }^{1} \mathrm{H} \mathrm{NMR}\left(\mathrm{CDCl}_{3}, 300 \mathrm{MHz}\right) \delta 7.24-7.14$ (m, 5H), 6.73-6.70 (m, 3H), 3.80 (s, 3H), 3.77 (s, 3H), 3.38 (s, 2H), 3.37 (s, 2H), 0.70 (s, 3H), 0.61 (s, 3H); ${ }^{13} \mathrm{C} \mathrm{NMR}\left(\mathrm{CDCl}_{3}, 75 \mathrm{MHz}\right) \delta 168.2,148.8,148.4,134.7,129.9,129.6,128.6,128.3,127.6,127.2,122.2$, 113.0, 111.1, 105.6, 60.0, 55.7, 44.8, 44.4, 28.7, 28.3; Anal. Calcd for $\mathrm{C}_{22} \mathrm{H}_{24} \mathrm{O}_{6}$ : C, 68.74; H, 6.29. Found: C, 68.52; H, 6.34 .

2-Benzyl-5,6-dimethoxy-1-indanone ${ }^{22}$ (77): Prepared from 76 in $80 \%$ yield using Procedure D in 45 min. Purified by flash chromatography (2:1 hexanes:EtOAc) to provide a white solid. M.p. $125-126^{\circ} \mathrm{C}$ $\left(\mathrm{CH}_{2} \mathrm{Cl}_{2}\right)$. Lit. $125-126{ }^{\circ} \mathrm{C} ;{ }^{1} \mathrm{H}$ NMR $\left(\mathrm{CDCl}_{3}, 300 \mathrm{MHz}\right) \delta$ 7.28-7.16 (m, 5H), $7.16(\mathrm{~s}, 1 \mathrm{H}), 6.77(\mathrm{~s}, 1 \mathrm{H})$, $3.89(\mathrm{~s}, 3 \mathrm{H}), 3.87(\mathrm{~s}, 3 \mathrm{H}), 3.33(\mathrm{dd}, J=14.0,4.0 \mathrm{~Hz}, 1 \mathrm{H}), 3.07-2.94(\mathrm{~m}, 2 \mathrm{H}), 2.73(\mathrm{dd}, J=16.3,2.7 \mathrm{~Hz}$, 1H), $2.60(\mathrm{dd}, J=13.9,10.0 \mathrm{~Hz}, 1 \mathrm{H}) ;{ }^{13} \mathrm{C} \mathrm{NMR}\left(\mathrm{CDCl}_{3}, 75 \mathrm{MHz}\right) \delta 206.3,155.6,149.5,148.8,139.7$, 
129.2, 128.8, 128.4, 126.2, 107.4, 104.4, 56.1, 56.0, 49.1, 37.2, 31.8; HRMS $m / z$ calcd for $\mathrm{C}_{18} \mathrm{H}_{18} \mathrm{O}_{3}\left(\mathrm{M}^{+}\right)$: 282.1256. Found: 282.1256.

5-Allyl-5-(3,4-dimethoxybenzyl)-2,2-dimethyl-1,3-dioxane-4,6-dione (78): The alkylation of 27 according to Procedure B using allyl bromide provided a white crystals in $67 \%$ yield following recrystallization from EtOH. M.p. 81-82 ${ }^{\circ} \mathrm{C}(\mathrm{EtOH}) ;{ }^{1} \mathrm{H} \mathrm{NMR}\left(\mathrm{CDCl}_{3}, 300 \mathrm{MHz}\right) \delta 6.72-6.64(\mathrm{~m}, 3 \mathrm{H})$, 5.70-5.56 (m, 1H), 5.19-5.11 (m, 2H), $3.77(\mathrm{~s}, 3 \mathrm{H}), 3.76(\mathrm{~s}, 3 \mathrm{H}), 3.21(\mathrm{~s}, 2 \mathrm{H}), 2.78(\mathrm{~d}, J=7.4 \mathrm{~Hz}, 2 \mathrm{H})$, $1.48(\mathrm{~s}, 3 \mathrm{H}), 0.73(\mathrm{~s}, 3 \mathrm{H}) ;{ }^{13} \mathrm{C} \mathrm{NMR}\left(\mathrm{CDCl}_{3}, 75 \mathrm{MHz}\right) \delta 168.4,148.9,148.5,130.5,127.5,122.3,121.2$, 113.1, 111.2, 105.7, 58.0, 55.8, 44.2, 43.3, 29.4, 29.0; Anal. Calcd for $\mathrm{C}_{18} \mathrm{H}_{22} \mathrm{O}_{6}$ : C, 64.66; H, 6.63. Found: C, 64.46; H, 6.73.

2-Allyl-5,6-dimethoxy-1-indanone (79): Prepared from 78 in 76\% yield using Procedure D in 45 min. Purified by flash chromatography (2:1 hexanes:EtOAc) to provide a pale yellow oil. ${ }^{1} \mathrm{H}$ NMR $\left(\mathrm{CDCl}_{3}, 300 \mathrm{MHz}\right) \delta 7.07(\mathrm{~s}, 1 \mathrm{H}), 6.78(\mathrm{~s}, 1 \mathrm{H}), 5.77-5.63(\mathrm{~m}, 1 \mathrm{H}), 5.01(\mathrm{~d}, J=17.0 \mathrm{~Hz}, 1 \mathrm{H}), 4.94(\mathrm{~d}, J=$ $10.0 \mathrm{~Hz}, 1 \mathrm{H}), 3.87(\mathrm{~s}, 3 \mathrm{H}), 3.81(\mathrm{~s}, 3 \mathrm{H}), 3.09$ (dd, $J=17.7,7.9 \mathrm{~Hz}, 1 \mathrm{H}), 2.71-2.55(\mathrm{~m}, 3 \mathrm{H}), 2.20-2.10$ (m, $1 \mathrm{H}) ;{ }^{13} \mathrm{C} \mathrm{NMR}\left(\mathrm{CDCl}_{3}, 75 \mathrm{MHz}\right) \delta 206.4,155.4,149.3,148.8,135.4,129.2,116.6,107.3,104.2,56.0$, 55.9, 46.6, 35.6, 31.6; HRMS $m / z$ calcd for $\mathrm{C}_{14} \mathrm{H}_{16} \mathrm{O}_{3}\left(\mathrm{M}^{+}\right):$232.1099. Found: 232.1093 .

5-(3,4-Dimethoxybenzyl)-2,2-dimethyl-5-(2-propynyl)-1,3-dioxane-4,6-dione (80): Prepared by alkylation of 27 according to Procedure B using propargyl bromide. Recrystallization from $\mathrm{MeOH}$ gave a white powder in $75 \%$ yield. M.p. $121-122{ }^{\circ} \mathrm{C}(\mathrm{MeOH}) ;{ }^{1} \mathrm{H} \mathrm{NMR}\left(\mathrm{CDCl}_{3}, 300 \mathrm{MHz}\right) \delta 6.74-6.60(\mathrm{~m}, 3 \mathrm{H})$, $3.79(\mathrm{~s}, 3 \mathrm{H}), 3.78(\mathrm{~s}, 3 \mathrm{H}), 3.17(\mathrm{~s}, 2 \mathrm{H}), 2.95(\mathrm{~d}, J=2.7 \mathrm{~Hz}, 2 \mathrm{H}), 2.12(\mathrm{t}, J=2.6 \mathrm{~Hz}, 1 \mathrm{H}), 1.61(\mathrm{~s}, 3 \mathrm{H}) 0.81$ $(\mathrm{s}, 3 \mathrm{H}) ;{ }^{13} \mathrm{C} \mathrm{NMR}\left(\mathrm{CDCl}_{3}, 75 \mathrm{MHz}\right) \delta$ 167.6, 148.8, 148.6, 126.4, 122.1, 112.7, 111.1, 106.3, 78.0, 72.9, 56.7, 55.7, 55.7, 44.0, 29.9, 28.4, 28.1; HRMS(EI) $m / z$ calcd for $\mathrm{C}_{18} \mathrm{H}_{20} \mathrm{O}_{6}\left(\mathrm{M}^{+}\right)$: 332.1260. Found: 332.1251.

5,6-Dimethoxy-2-(2-propynyl)-1-indanone (81): Prepared from 80 in $80 \%$ yield using Procedure D in 45 min. Purified by flash chromatography (2:1 hexanes:EtOAc) to provide a white solid. M.p. 109-111 ${ }^{\circ} \mathrm{C}\left(\mathrm{CH}_{2} \mathrm{Cl}_{2}\right) ;{ }^{1} \mathrm{H}$ NMR $\left(\mathrm{CDCl}_{3}, 300 \mathrm{MHz}\right) \delta 7.09$ (s, 1H), 6.83 (s, 1H), $3.90(\mathrm{~s}, 3 \mathrm{H}), 3.83$ (s, $\left.3 \mathrm{H}\right), 3.22$ $(\mathrm{dd}, J=17.0,7.5 \mathrm{~Hz}, 1 \mathrm{H}), 2.92(\mathrm{dd}, J=17.0,3.6 \mathrm{~Hz}, 1 \mathrm{H}), 2.77-2.64(\mathrm{~m}, 2 \mathrm{H}), 2.45(\mathrm{ddd}, J=16.6,7.9 \mathrm{~Hz}$, $2.5 \mathrm{~Hz}), 1.83(\mathrm{t}, J=2.1 \mathrm{~Hz}, 1 \mathrm{H}) ;{ }^{13} \mathrm{C} \mathrm{NMR}\left(\mathrm{CDCl}_{3}, 75 \mathrm{MHz}\right) \delta 204.7,155.6,149.4,148.8,129.0,107.3$, 104.3, 81.2, 69.3, 56.1, 55.9, 45.7, 31.6, 20.1; HRMS $m / z$ calcd for $\mathrm{C}_{14} \mathrm{H}_{14} \mathrm{O}_{3}\left(\mathrm{M}^{+}\right)$: 230.0943. Found: 230.0948 . 


\section{4-[5-(3,4-Dimethoxybenzyl)-2,2-dimethyl-4,6-dioxo-1,3-dioxan-5-yl]methylbenzonitrile (82):}

The alkylation of 27 according to Procedure B using $\alpha$-bromo- $p$-tolunitrile provided a $79 \%$ yield of white powder following recrystallization from $\mathrm{MeOH}$. M.p. $158-159.5{ }^{\circ} \mathrm{C}(\mathrm{MeOH}) ;{ }^{1} \mathrm{H}$ NMR $\left(\mathrm{CDCl}_{3}, 300\right.$ MHz) $\delta 7.49(\mathrm{~d}, J=8.2 \mathrm{~Hz}, 2 \mathrm{H}), 7.21(\mathrm{~d}, J=8.2 \mathrm{~Hz}, 2 \mathrm{H}), 6.67-6.60(\mathrm{~m}, 3 \mathrm{H}), 3.72(\mathrm{~s}, 3 \mathrm{H}), 3.71(\mathrm{~s}, 3 \mathrm{H})$, $3.37(\mathrm{~s}, 2 \mathrm{H}), 3.31(\mathrm{~s}, 2 \mathrm{H}), 0.65(\mathrm{~s}, 3 \mathrm{H}), 0.60(\mathrm{~s}, 3 \mathrm{H}) ;{ }^{13} \mathrm{C} \mathrm{NMR}\left(\mathrm{CDCl}_{3}, 75 \mathrm{MHz}\right) \delta 167.7,148.8,148.5$, $140.0,132.2,130.7,126.5,122.1,118.1,112.8,111.6,111.1,105.8,59.5,55.6,44.5,44.3,28.6,28.5$; HRMS(EI) $m / z$ calcd for $\mathrm{C}_{23} \mathrm{H}_{23} \mathrm{NO}_{6}\left(\mathrm{M}^{+}\right): 409.1525$. Found: 409.1531 .

4-[(5,6-Dimethoxy-1-oxo-2,3-dihydro-1H-2-indenyl)methyl]benzonitrile (83): Prepared from 82 in $78 \%$ yield using Procedure D with $17 \mathrm{~mol} \% \mathrm{Sc}(\mathrm{OTf})_{3}$ in $320 \mathrm{~min}$. Purified by flash chromatography (3:1 hexanes:EtOAc) to provide a pale yellow solid. Recrystallization from methanol afforded clear colorless crystals. M.p. 259-260 ${ }^{\circ} \mathrm{C}(\mathrm{MeOH}) ; \mathrm{IR}\left(\right.$ neat) $2224 \mathrm{~cm}^{-1} ;{ }^{1} \mathrm{H}$ NMR $\left(\mathrm{CDCl}_{3}, 300 \mathrm{MHz}\right) \delta 7.55$ (d, $J=8.2 \mathrm{~Hz}, 2 \mathrm{H}), 7.32(\mathrm{~d}, J=8.1 \mathrm{~Hz}, 2 \mathrm{H}), 7.15(\mathrm{~s}, 1 \mathrm{H}), 6.78(\mathrm{~s}, 1 \mathrm{H}), 3.91(\mathrm{~s}, 1 \mathrm{H}), 3.88(\mathrm{~s}, 3 \mathrm{H}), 3.35$ (dd, $J$ $=13.9,4.2 \mathrm{~Hz}, 1 \mathrm{H}), 3.07(\mathrm{dd}, J=16.7,7.5 \mathrm{~Hz}, 1 \mathrm{H}), 2.99-2.91(\mathrm{~m}, 1 \mathrm{H}), 2.79-2.63(\mathrm{~m}, 2 \mathrm{H}) ;{ }^{13} \mathrm{C} \mathrm{NMR}$ $\left(\mathrm{CDCl}_{3}, 75 \mathrm{MHz}\right) \delta 155.8,149.7,148.5,145.4,132.3,129.7,129.0,118.9,110.3,107.3,104.4,56.2,56.1$, 48.4, 37.2, 31.7; HRMS(EI) $m / z$ calcd for $\mathrm{C}_{19} \mathrm{H}_{17} \mathrm{NO}_{3}\left(\mathrm{M}^{+}\right)$: 307.1208. Found: 307.1211.

5-(3,4-Dimethoxybenzyl)-2,2-dimethyl-5-(4-nitrobenzyl)-1,3-dioxane-4,6-dione (84): Compound 27 was alkylated according to Procedure B using 4-nitrobenzyl bromide and recrystallized from $\mathrm{MeOH}$ to provide a white solid in $81 \%$ yield. M.p. $192-193{ }^{\circ} \mathrm{C}(\mathrm{MeOH}) ;{ }^{1} \mathrm{H}$ NMR $\left(\mathrm{CDCl}_{3}, 300\right.$ MHz) $\delta 8.09(\mathrm{~d}, J=8.7 \mathrm{~Hz}, 2 \mathrm{H}), 7.32(\mathrm{~d}, J=8.7 \mathrm{~Hz}, 2 \mathrm{H}), 6.71-6.64(\mathrm{~m}, 3 \mathrm{H}), 3.78$ (s, 3H), $3.76(\mathrm{~s}, 3 \mathrm{H})$, $3.46(\mathrm{~s}, 3 \mathrm{H}), 3.37(\mathrm{~s}, 2 \mathrm{H}), 0.71(\mathrm{~s}, 3 \mathrm{H}), 0.64(\mathrm{~s}, 3 \mathrm{H}) ;{ }^{13} \mathrm{C} \mathrm{NMR}\left(\mathrm{CDCl}_{3}, 75 \mathrm{MHz}\right) \delta$ 167.8, 148.9, 148.7, $142.4,142.1,131.0,126.6,123.7,122.3,112.9,111.2,106.0,59.5,55.8,55.7,44.5,44.3,28.9,28.6$; HRMS(EI) $m / z$ calcd for $\mathrm{C}_{22} \mathrm{H}_{23} \mathrm{NO}_{8}\left(\mathrm{M}^{+}\right)$: 429.1424. Found: 429.1418.

5,6-Dimethoxy-2-(4-nitrobenzyl)-1-indanone (85): Prepared from 84 in $81 \%$ yield using Procedure D in 250 min. Purified by flash chromatography (4:1 hexanes:EtOAc) to provide a pale yellow solid. Recrystallization from MeOH afforded clear, colorless crystals. M.p. $188-190{ }^{\circ} \mathrm{C}(\mathrm{MeOH})$; IR(neat) 2361 $\mathrm{cm}^{-1} ;{ }^{1} \mathrm{H} \mathrm{NMR}\left(\mathrm{CDCl}_{3}, 300 \mathrm{MHz}\right) \delta 8.15(\mathrm{~d}, J=8.6 \mathrm{~Hz}, 2 \mathrm{H}), 7.40(\mathrm{~d}, J=8.5 \mathrm{~Hz}, 2 \mathrm{H}), 7.18(\mathrm{~s}, 1 \mathrm{H}), 6.82$ (s, 1H), $3.94(\mathrm{~s}, 3 \mathrm{H}), 3.91(\mathrm{~s}, 3 \mathrm{H}), 3.42(\mathrm{dd}, J=13.8,4.1 \mathrm{~Hz}, 1 \mathrm{H}), 3.12$ (dd, $J=16.6,7.5 \mathrm{~Hz}, 1 \mathrm{H}), 3.16-$ $2.97(\mathrm{~m}, 1 \mathrm{H}), 2.86(\mathrm{dd}, J=13.8,9.5 \mathrm{~Hz}, 1 \mathrm{H}), 2.73(\mathrm{dd}, J=16.7,3.2 \mathrm{~Hz}, 1 \mathrm{H}) ;{ }^{13} \mathrm{C} \mathrm{NMR}\left(\mathrm{CDCl}_{3}, 75\right.$ 
MHz) § 205.3. 155.8, 149.6, 148.4, 147.5, 146.7, 129.7, 128.9, 123.7, 107.3, 104.4, 56.2, 56.1, 48.3, 36.9, 31.6; $\mathrm{HRMS}(\mathrm{EI}) \mathrm{m} / z$ calcd for $\mathrm{C}_{18} \mathrm{H}_{17} \mathrm{NO}_{5}\left(\mathrm{M}^{+}\right)$: 327.1107. Found: 327.1105 .

\section{5-(3,4-Dimethoxybenzyl)-2,2-dimethyl-5-(2,3,4,5,6-pentafluorobenzyl)-1,3-dioxane-4,6-dione}

(86): Compound 27 was alkylated according to Procedure B using 2,3,4,5,6-pentafluorobenzyl bromide and recrystallized from $\mathrm{MeOH}$ to provide a white powder in $95 \%$ yield. M.p. $169-169.5{ }^{\circ} \mathrm{C}(\mathrm{MeOH}) ;{ }^{1} \mathrm{H}$ $\operatorname{NMR}\left(\mathrm{CDCl}_{3}, 300 \mathrm{MHz}\right) \delta$ 6.75-6.66 (m, 3H), 3.80 (s, 6H), 3.44 (s, 2H), 3.35 (s, 2H), 1.50 (s, 3H), 0.85 $(\mathrm{s}, 3 \mathrm{H}) ;{ }^{13} \mathrm{C} \mathrm{NMR}\left(\mathrm{CDCl}_{3}, 75 \mathrm{MHz}\right) \delta$ 167.5, 148.8, 148.5, $147.2(\mathrm{~m}), 143.9(\mathrm{~m}), 139.0(\mathrm{~m}), 135.6(\mathrm{~m})$, 126.6, 122.7, 113.3, 111.0, 108.5 (m), 105.8, 56.4, 55.6, 41.7, 31.9, 28.9, 28.6; HRMS(EI) $m / z$ calcd for $\mathrm{C}_{22} \mathrm{H}_{19} \mathrm{~F}_{5} \mathrm{O}_{6}\left(\mathrm{M}^{+}\right)$: 474.1102. Found: 474.1108.

5,6-Dimethoxy-2-(2,3,4,5,6-pentafluorobenzyl)-1-indanone (87): Prepared from 86 in $80 \%$ yield using Procedure D in $85 \mathrm{~min}$. Purified by flash chromatography (6:1 hexanes:EtOAc) to provide a white solid. M.p. 163-164 ${ }^{\circ} \mathrm{C}\left(\mathrm{CH}_{2} \mathrm{Cl}_{2}\right) ;{ }^{1} \mathrm{H} \mathrm{NMR}\left(\mathrm{CDCl}_{3}, 300 \mathrm{MHz}\right) \delta 7.17$ (s, 1H), 6.83 (s, 1H), 3.95 (s, 3H), $3.90(\mathrm{~s}, 3 \mathrm{H}), 3.29(\mathrm{dd}, J=13.7 \mathrm{~Hz}, 5.4 \mathrm{~Hz}, 1 \mathrm{H}), 3.14(\mathrm{dd}, J=16.8,7.5 \mathrm{~Hz}, 1 \mathrm{H}), 2.97-2.94(\mathrm{~m}, 1 \mathrm{H}), 2.90-$ $2.82(\mathrm{~m}, 2 \mathrm{H}) ;{ }^{13} \mathrm{C} \mathrm{NMR}\left(\mathrm{CDCl}_{3}, 75 \mathrm{MHz}\right) \delta 204.3,155.8,149.6,148.1,146.8(\mathrm{~m}), 143.6(\mathrm{~m}), 141.5(\mathrm{~m})$, 139.3-138.2 (m), 135.9-135.7 (m), 128.6, $113.1(\mathrm{~m}), 107.3,104.4,56.1$ (d, $J=7.5 \mathrm{~Hz}), 46.7(\mathrm{~d}, J=15.0$ $\mathrm{Hz}), 32.1,24.3$; $\mathrm{HRMS}(\mathrm{EI}) \mathrm{m} / z$ calcd for $\mathrm{C}_{18} \mathrm{H}_{13} \mathrm{~F}_{5} \mathrm{O}_{3}\left(\mathrm{M}^{+}\right): 372.0785$. Found: 372.0782 .

\section{5-[1-(3,5-Dimethoxyphenyl)ethyl]-2,2,5-trimethyl-1,3-dioxane-4,6-dione (88): Alkylation of 11} according to Procedure B using iodomethane, followed by flash chromatography (4:1 hexanes:EtOAc) and recrystallization from $\mathrm{MeOH}$ gave a $60 \%$ yield of a white solid. M.p. $72-73{ }^{\circ} \mathrm{C}(\mathrm{MeOH}) ;{ }^{1} \mathrm{H}$ NMR $\left(\mathrm{CDCl}_{3}, 300 \mathrm{MHz}\right) \delta 6.30(\mathrm{~s}, 3 \mathrm{H}), 3.71(\mathrm{~s}, 6 \mathrm{H}), 3.41$ (q, J = 7.2 Hz, 1H), 1.63 (s, 3H), $1.54(\mathrm{~s}, 3 \mathrm{H}), 1.51$ $(\mathrm{d}, J=7.2 \mathrm{~Hz}, 3 \mathrm{H}), 1.04(\mathrm{~s}, 3 \mathrm{H}) ;{ }^{13} \mathrm{C} \mathrm{NMR}\left(\mathrm{CDCl}_{3}, 75 \mathrm{MHz}\right) \delta 170.4,168.6,160.7,142.7,106.5,104.9$, 100.0, 55.3, 54.0, 48.6, 30.0, 27.6, 22.5, 15.2; $\mathrm{HRMS}(\mathrm{EI}) \mathrm{m} / \mathrm{z}$ calcd for $\mathrm{C}_{17} \mathrm{H}_{22} \mathrm{O}_{6}\left(\mathrm{M}^{+}\right)$: 322.1416. Found: 322.1424.

5,7-Dimethoxy-2,3-dimethyl-1-indanone ${ }^{23}(\mathbf{8 9})$ : Prepared from 88 in 91\% yield as a 5.6:1 mixture of trans:cis isomers using Procedure D in 155 min. Purified by flash chromatography (6:1 hexanes:EtOAc) to provide a mixture of trans:cis isomers (5.6:1) as a clear, colorless oil. Trans isomer: ${ }^{1} \mathrm{H}$ NMR $\left(\mathrm{CDCl}_{3}, 300 \mathrm{MHz}\right) \delta 6.42(\mathrm{~s}, 1 \mathrm{H}), 6.26$ (s, 1H), 3.85 (s, 3H), $3.84(\mathrm{~s}, 3 \mathrm{H}), 2.71-2.77(\mathrm{~m}, 1 \mathrm{H})$, 2.11-2.19 (m, 1H), $1.33(\mathrm{~d}, J=7.1 \mathrm{~Hz}, 3 \mathrm{H}), 1.19(J=6.1 \mathrm{~Hz}, 3 \mathrm{H}) ;{ }^{13} \mathrm{C} \mathrm{NMR}\left(\mathrm{CDCl}_{3}, 75 \mathrm{MHz}\right) \delta 204.4$, 167.0, 162.9, 159.1, 117.8, 100.2, 97.2, 55.7, 55.6, 51.5, 41.6, 19.4, 15.0. Cis isomer: ${ }^{1} \mathrm{H}$ NMR $\left(\mathrm{CDCl}_{3}\right.$, 
$300 \mathrm{MHz}) \delta 6.42(\mathrm{~s}, 1 \mathrm{H}), 6.26(\mathrm{~s}, 1 \mathrm{H}), 3.85(\mathrm{~s}, 3 \mathrm{H}), 3.84(\mathrm{~s}, 3 \mathrm{H}), 3.35-3.29(\mathrm{~m}, 1 \mathrm{H}), 2.19-2.11(\mathrm{~m}, 1 \mathrm{H})$, $1.16(\mathrm{~d}, J=7.3 \mathrm{~Hz}, 3 \mathrm{H}), 1.12(J=7.6 \mathrm{~Hz}, 3 \mathrm{H}) ;{ }^{13} \mathrm{C} \mathrm{NMR}\left(\mathrm{CDCl}_{3}, 75 \mathrm{MHz}\right) \delta 205.1,166.8,164.2,159.1$, 117.4, 100.6, 97.2, 55.7, 55.6, 46.6, 36.6, 16.8; HRMS(EI) $m / z$ calcd for $\mathrm{C}_{13} \mathrm{H}_{16} \mathrm{O}_{3}\left(\mathrm{M}^{+}\right)$(mixture of isomers): 220.1099. Found: 220.1095.

5-Benzyl-5-[1-(3,5-dimethoxyphenyl)ethyl]-2,2-dimethyl-1,3-dioxane-4,6-dione (90): Alkylation of 11 according to Procedure B using benzyl bromide, followed by flash chromatography (4:1 hexanes:EtOAc) and recrystallization from $\mathrm{MeOH}$ gave a $67 \%$ yield of a white powder. M.p. $125-126^{\circ} \mathrm{C}$ $(\mathrm{MeOH}) ;{ }^{1} \mathrm{H}$ NMR $\left(\mathrm{CDCl}_{3}, 300 \mathrm{MHz}\right)$ d 7.24-7.13 (m, 5H), 6.32-6.29 (m, 3H), 3.69 (s, 6H), 3.57 (q, $J=$ $7.2 \mathrm{~Hz}, 1 \mathrm{H}), 3.55(\mathrm{~d}, J=12.5 \mathrm{~Hz}, 1 \mathrm{H}), 3.18(\mathrm{~d}, J=12.5 \mathrm{~Hz}, 1 \mathrm{H}), 1.62(\mathrm{~d}, J=7.2 \mathrm{~Hz}, 3 \mathrm{H}), 0.67(\mathrm{~s}, 3 \mathrm{H})$, $0.48(\mathrm{~s}, 3 \mathrm{H}) ;{ }^{13} \mathrm{C} \mathrm{NMR}\left(\mathrm{CDCl}_{3}, 75 \mathrm{MHz}\right) \delta 168.5,166.8,160.7,142.5,135.2,130.5,128.6,127.5,106.4$, 105.6, 99.9, 61.8, 55.2, 48.7, 41.5, 28.9, 27.8, 15.5; HRMS(EI) $m / z$ calcd for $\mathrm{C}_{23} \mathrm{H}_{26} \mathrm{O}_{6}\left(\mathrm{M}^{+}\right): 398.1729$. Found: 398.1728.

2-Benzyl-5,7-dimethoxy-3-methyl-1-indanone (91): Prepared from 90 in 92\% yield as a 12.3:1 mixture of trans:cis isomers using Procedure D with 17 mol \% Sc(OTf) 3 in 32 hours. Purified by flash chromatography (6:1 hexanes:EtOAc) to provide a mixture of trans:cis isomers $(12.3: 1)$ as a pale yellow oil. Trans isomer: ${ }^{1} \mathrm{H}$ NMR $\left(\mathrm{CDCl}_{3}, 300 \mathrm{MHz}\right) \delta$ 7.29-7.16 (m, 1H), $6.34(\mathrm{~s}, 1 \mathrm{H}), 6.29(\mathrm{~s}, 1 \mathrm{H}), 3.88(\mathrm{~s}$, 3H), $3.83(\mathrm{~s}, 3 \mathrm{H}), 3.33(\mathrm{dd}, J=13.6,4.3 \mathrm{~Hz}, 1 \mathrm{H}), 2.92-2.96(\mathrm{~m}, 1 \mathrm{H}), 2.67$ (dd, $J=13.6,9.9 \mathrm{~Hz}, 1 \mathrm{H}), 2.46-$ $2.51(\mathrm{~m}, 1 \mathrm{H}), 1.06(\mathrm{~d}, J=7.1 \mathrm{~Hz}, 3 \mathrm{H}) ;{ }^{13} \mathrm{C} \mathrm{NMR}\left(\mathrm{CDCl}_{3}, 75 \mathrm{MHz}\right) \delta 202.7,167.0,163.2,159.1,139.7$, 128.9, 128.2, 126.0, 117.7, 100.2, 97.2, 57.9, 55.6, 55.5, 38.3, 36.5, 20.1. Cis isomer: ${ }^{1} \mathrm{H}$ NMR $\left(\mathrm{CDCl}_{3}\right.$, $300 \mathrm{MHz}) \delta$ 7.29-7.16 (m, 1H), 6.47 (s, 1H), $6.44(\mathrm{~s}, 1 \mathrm{H}), 3.88$ (s, 3H), 3.83 (s, 3H), 3.33 (dd, $J=13.6$, $4.3 \mathrm{~Hz}, 1 \mathrm{H}), 2.96-2.92(\mathrm{~m}, 1 \mathrm{H}), 2.67(\mathrm{dd}, J=13.6,9.9 \mathrm{~Hz}, 1 \mathrm{H}), 2.51-2.46(\mathrm{~m}, 1 \mathrm{H}), 1.13(\mathrm{~d}, J=7.2 \mathrm{~Hz}$, $3 \mathrm{H}) ;{ }^{13} \mathrm{C} \mathrm{NMR}\left(\mathrm{CDCl}_{3}, 75 \mathrm{MHz}\right) \delta 202.4,166.7,164.0,140.5,128.9,127.9,125.7,117.0,100.7,97.2$, 60.2, 55.0, 52.9, 31.1, 20.9, 18.7, 14.0; $\mathrm{HRMS}(\mathrm{EI}) \mathrm{m} / \mathrm{z}$ calcd for $\mathrm{C}_{19} \mathrm{H}_{20} \mathrm{O}_{3}\left(\mathrm{M}^{+}\right)$(mixture of isomers): 296.1412. Found: 296.1407.

5-(2,5-Dimethoxybenzyl)-2,2,5-trimethyl-1,3-dioxane-4,6-dione (92): Alkylation of 32 according to Procedure B using iodomethane produced a white solid in $61 \%$ yield after recrystallization from $\mathrm{MeOH}$. M.p. 76-77 ${ }^{\circ} \mathrm{C}(\mathrm{MeOH}) ;{ }^{1} \mathrm{H} \mathrm{NMR}\left(\mathrm{CDCl}_{3}, 300 \mathrm{MHz}\right) \delta$ 6.75-6.67(3H, m), $3.71(3 \mathrm{H}, \mathrm{s}), 3.69(3 \mathrm{H}, \mathrm{s}), 3.27$ $(2 \mathrm{H}, \mathrm{s}), 1.66(3 \mathrm{H}, \mathrm{s}), 1.61(3 \mathrm{H}, \mathrm{s}), 1.32(3 \mathrm{H}, \mathrm{s}) ;{ }^{13} \mathrm{C} \mathrm{NMR}\left(\mathrm{CDCl}_{3}, 75 \mathrm{MHz}\right) \delta 169.4,153.3,124.0,117.8$, 
113.9, 111.3, 104.8, 55.7, 55.4, 50.1, 40.8, 30.0, 27.9, 23.7; $\mathrm{HRMS}(\mathrm{EI}) \mathrm{m} / z$ calcd for $\mathrm{C}_{16} \mathrm{H}_{20} \mathrm{O}_{6}\left(\mathrm{M}^{+}\right)$: 308.1260. Found: 308.1259.

4,7-Dimethoxy-2-methyl-1-indanone ${ }^{24}$ (93): Prepared from 92 in 69\% yield using Procedure D in 20 min. Purified by flash chromatography (5:1 hexanes:EtOAc) to provide a white solid. M.p. $78-79{ }^{\circ} \mathrm{C}$ $\left(\mathrm{Et}_{2} \mathrm{O}\right)$. Lit. $79.5-82.5^{\circ} \mathrm{C} ;{ }^{1} \mathrm{H} \mathrm{NMR}\left(\mathrm{CDCl}_{3}, 300 \mathrm{MHz}\right) \delta 6.89(\mathrm{~d}, J=8.7 \mathrm{~Hz}, 1 \mathrm{H}), 6.64(\mathrm{~d}, J=8.7 \mathrm{~Hz}$, 1H), $3.81(\mathrm{~s}, 3 \mathrm{H}), 3.76(\mathrm{~s}, 3 \mathrm{H}), 3.16(\mathrm{dd}, J=17.5,7.7 \mathrm{~Hz}, 1 \mathrm{H}), 2.59-2.43(\mathrm{~m}, 2 \mathrm{H}), 1.19(\mathrm{~d}, J=7.4 \mathrm{~Hz}$, $3 \mathrm{H}) ;{ }^{13} \mathrm{C} \mathrm{NMR}\left(\mathrm{CDCl}_{3}, 75 \mathrm{MHz}\right) \delta 207.2,151.8,150.3,144.1,125.4,116.5,109.5,55.8,42.0,42.1,31.2$, 16.7; HRMS(EI) $m / z$ calcd for $\mathrm{C}_{12} \mathrm{H}_{14} \mathrm{O}_{3}\left(\mathrm{M}^{+}\right)$: 206.0943. Found: 206.0937 .

\section{5-(2-[1-(tert-Butyl)-1,1-diphenylsilyl]oxy-5-methoxybenzyl)-2,2,5-trimethyl-1,3-dioxane-4,6-}

dione (94): Alkylation of $\mathbf{3 4}$ according to Procedure B using iodomethane was followed by flash chromatography using 5:1 (hexanes: EtOAc) to afford a pale yellow oil. ${ }^{1} \mathrm{H}$ NMR $\left(\mathrm{CDCl}_{3}, 300 \mathrm{MHz}\right)$ ઈ 7.75-7.70 (m, 4H), 7.40-7.34 (m, 6H), 6.69-6.68 (m, 1H), 6.31-6.30 (m, 2H), $3.62(\mathrm{~s}, 3 \mathrm{H}), 3.48(\mathrm{~s}, 2 \mathrm{H})$, $1.78(\mathrm{~s}, 3 \mathrm{H}), 1.66(\mathrm{~s}, 3 \mathrm{H}), 1.33(\mathrm{~s}, 3 \mathrm{H}), 1.14(\mathrm{~s}, 9 \mathrm{H}) ;{ }^{13} \mathrm{C} \mathrm{NMR}\left(\mathrm{CDCl}_{3}, 75 \mathrm{MHz}\right) \delta 169.7,153.1,147.5$, $135.3,132.5,129.7,127.6,125.2,119.9,115.7,113.6,104.9,55.3,50.6,39.5,30.0,27.8,26.4,23.8,19.2$; HRMS(EI) $m / z$ calcd for $\mathrm{C}_{31} \mathrm{H}_{36} \mathrm{O}_{6} \mathrm{Si}\left(\mathrm{M}^{+}\right)$: 532.2281. Found: 532.2278.

4-[1-(tert-Butyl)-1,1-diphenylsilyl]oxy-7-methoxy-2-methyl-1-indanone (95): Prepared from 94 in $94 \%$ yield using Procedure D in 20 min. Purified by flash chromatography (4:1 hexanes:EtOAc) to provide a pale yellow oil. ${ }^{1} \mathrm{H}$ NMR $\left(\mathrm{CDCl}_{3}, 300 \mathrm{MHz}\right) \delta 7.67(\mathrm{~d}, J=6.5 \mathrm{~Hz}, 4 \mathrm{H}), 7.45-7.33(\mathrm{~m}, 6 \mathrm{H}), 6.61$ $(\mathrm{d}, J=8.7 \mathrm{~Hz}, 1 \mathrm{H}), 6.36(\mathrm{~d}, J=8.7 \mathrm{~Hz}, 1 \mathrm{H}), 3.77(\mathrm{~s}, 3 \mathrm{H}), 3.37-3.29(\mathrm{~m}, \mathrm{~A}$ of AB, 1H), $2.65(\mathrm{~m}, 1 \mathrm{H}), 2.56$ $(\mathrm{m}, \mathrm{B}$ of $\mathrm{AB}, 1 \mathrm{H}), 1.26(3 \mathrm{H}, \mathrm{d}, J=7.3 \mathrm{~Hz}), 1.11(\mathrm{~s}, 9 \mathrm{H}) ;{ }^{13} \mathrm{C} \mathrm{NMR}\left(\mathrm{CDCl}_{3}, 75 \mathrm{MHz}\right) \delta 207.3,152.2$, $146.1,145.5,135.4,132.5,130.1,127.9,125.2,125.1,109.4,55.8,42.1,31.9,26.5,19.5,16.8 ;$ HRMS(EI) $m / z$ calcd for $\mathrm{C}_{27} \mathrm{H}_{30} \mathrm{O}_{3} \mathrm{Si}\left(\mathrm{M}^{+}\right)$: 430.1964. Found: 430.1967 .

5-(2,3-Dimethoxybenzyl)-2,2,5-trimethyl-1,3-dioxane-4,6-dione (96): Alkylation of 36 according to Procedure B using iodomethane, followed by flash chromatography (6:1 hexanes:EtOAc) produced a $50 \%$ yield of white solid. M.p. $72-73{ }^{\circ} \mathrm{C}\left(\mathrm{CH}_{2} \mathrm{Cl}_{2}\right) ;{ }^{1} \mathrm{H} \mathrm{NMR}\left(\mathrm{CDCl}_{3}, 300 \mathrm{MHz}\right) \delta 6.92(\mathrm{t}, J=7.8 \mathrm{~Hz}$, $1 \mathrm{H}), 6.82(\mathrm{~d}, J=1.5 \mathrm{~Hz}, 1 \mathrm{H}), 6.73(\mathrm{~d}, J=1.5 \mathrm{~Hz}, 2 \mathrm{H}), 3.80(\mathrm{~s}, 3 \mathrm{H}), 3.79(\mathrm{~s}, 3 \mathrm{H}), 3.32(\mathrm{~s}, 3 \mathrm{H}), 1.69$ (s, $3 \mathrm{H}), 1.61(\mathrm{~s}, 3 \mathrm{H}) ;{ }^{13} \mathrm{C} \mathrm{NMR}\left(\mathrm{CDCl}_{3}, 75 \mathrm{MHz}\right) \delta 169.5,152.6,147.9,128.5,123.5,112.5,105.1,60.5$, 55.6, 50.3, 40.1, 29.9, 28.1, 24.6; HRMS(EI) $m / z$ calcd for $\mathrm{C}_{16} \mathrm{H}_{20} \mathrm{O}_{6}\left(\mathrm{M}^{+}\right)$: 308.1260. Found: 308.1249. 
4,5-Dimethoxy-2-methyl-1-indanone ${ }^{25}$ (97): Prepared from 96 in $75 \%$ yield using Procedure D in 20 min. Purified by flash chromatography (7:1 hexanes:EtOAc) to provide a white solid. M.p. $69-70{ }^{\circ} \mathrm{C}$ $\left(\mathrm{Et}_{2} \mathrm{O}\right) ;{ }^{1} \mathrm{H} \mathrm{NMR}\left(\mathrm{CDCl}_{3}, 300 \mathrm{MHz}\right) \delta 7.49(\mathrm{~d}, J=8.3 \mathrm{~Hz}, 1 \mathrm{H}), 6.94(\mathrm{~d}, J=8.4 \mathrm{~Hz}, 1 \mathrm{H}), 3.91(\mathrm{~s}, 3 \mathrm{H})$, $3.88(\mathrm{~s}, 3 \mathrm{H}), 3.36(\mathrm{dd}, J=18.2,8.8 \mathrm{~Hz}, 1 \mathrm{H}), 2.69-2.60(\mathrm{~m}, 2 \mathrm{H}), 1.25(\mathrm{~d}, J=7.2 \mathrm{~Hz}, 3 \mathrm{H}) ;{ }^{13} \mathrm{C} \mathrm{NMR}$ $\left(\mathrm{CDCl}_{3}, 75 \mathrm{MHz}\right) \delta 207.8,157.6,146.1,145.3,130.3,120.4,112.3,60.3,56.2,42.1,31.5,16.5 ;$ HRMS(EI) $m / z$ calcd for $\mathrm{C}_{12} \mathrm{H}_{14} \mathrm{O}_{3}\left(\mathrm{M}^{+}\right)$: 206.0943. Found: 206.0947 .

\section{5-(2-[1-(tert-Butyl)-1,1-diphenylsilyl] oxy-3-methoxybenzyl)-2,2,5-trimethyl-1,3-dioxane-4,6-}

dione (98): Alkylation of 42 according to Procedure B using iodomethane was followed by flash

chromatography (5:1 hexanes:EtOAc) and recrystallization from $\mathrm{MeOH}$ to give an $84 \%$ yield of white powder. M.p. $131-132{ }^{\circ} \mathrm{C}(\mathrm{MeOH}) ;{ }^{1} \mathrm{H} \mathrm{NMR}\left(\mathrm{CDCl}_{3}, 300 \mathrm{MHz}\right) \delta$ 7.68-7.65 (m, 4H), 7.33-7.27 (m, 6H), $6.71(\mathrm{t}, J=3.1 \mathrm{~Hz}, 2 \mathrm{H}), 6.39(\mathrm{dd}, J=6.1,3.5 \mathrm{~Hz}, 1 \mathrm{H}), 3.64(\mathrm{~s}, 2 \mathrm{H}), 2.73(\mathrm{~s}, 3 \mathrm{H}), 1.75(\mathrm{~s}, 3 \mathrm{H}), 1.66(\mathrm{~s}$, 3H), 1.35 (s, 3H), $1.10(\mathrm{~s}, 9 \mathrm{H}) ;{ }^{13} \mathrm{C} \mathrm{NMR}\left(\mathrm{CDCl}_{3}, 75 \mathrm{MHz}\right) \delta 169.7,149.4,143.6,135.0,134.3,128.8$, 127.1, 125.5, 121.9, 120.7, 110.7, 104.9, 52.4, 51.0, 37.0, 30.0, 28.0, 27.0, 24.3, 20.0; HRMS(EI) $\mathrm{m} / \mathrm{z}$ calcd for $\mathrm{C}_{31} \mathrm{H}_{36} \mathrm{O}_{6} \mathrm{Si}\left(\mathrm{M}^{+}-\mathrm{CH}_{3}\right): 517.2046$. Found: 517.2039.

4-[1-(tert-Butyl)-1,1-diphenylsilyl]oxy-5-methoxy-2-methyl-1-indanone (99): Prepared from 98 in $86 \%$ yield using Procedure D in 30 min. Purified by flash chromatography (11:1 hexanes:EtOAc) to provide a clear colorless oil. ${ }^{1} \mathrm{H}$ NMR $\left(\mathrm{CDCl}_{3}, 300 \mathrm{MHz}\right) \delta 7.84(\mathrm{dd}, J=6.3,1.2 \mathrm{~Hz}, 4 \mathrm{H}), 7.53-7.40(\mathrm{~m}$, $7 \mathrm{H}), 3.54(\mathrm{~d}, J=8.3 \mathrm{~Hz}, 1 \mathrm{H}), 3.54(\mathrm{q}, J=9.4 \mathrm{~Hz}, 1 \mathrm{H}), 3.25(\mathrm{~s}, 3 \mathrm{H}), 2.84-2.76(\mathrm{~m}, 2 \mathrm{H}), 1.43(\mathrm{~d}, J=7.2$ $\mathrm{Hz}, 3 \mathrm{H}), 1.27(\mathrm{~s}, 9 \mathrm{H}) ;{ }^{13} \mathrm{C} \mathrm{NMR}\left(\mathrm{CDCl}_{3}, 75 \mathrm{MHz}\right) \delta 208.3,154.6,144.0,141.4,134.7,134.1,130.2$, 129.4, 127.3, 117.9, 111.6, 54.4, 42.1, 32.2, 26.7, 20.0, 16.5; HRMS(EI) $m / z$ calcd for $\mathrm{C}_{27} \mathrm{H}_{30} \mathrm{O}_{3} \mathrm{Si}\left(\mathrm{M}^{+}-\right.$ $\left.\mathrm{CH}_{3}\right):$ 415.1724. Found: 415.1735.

\section{5-(3-Methoxy-2-[(1,1,1-triisopropylsilyl)oxy]benzyl)-2,2,5-trimethyl-1,3-dioxane-4,6-dione}

(100): Alkylation of 23 according to Procedure B using iodomethane, followed by flash chromatography (8:1 hexanes:EtOAc) provided a white solid. M.p. 60-61 ${ }^{\circ} \mathrm{C}$ (Benzene); ${ }^{1} \mathrm{H}$ NMR $\left(\mathrm{CDCl}_{3}, 300 \mathrm{MHz}\right)$

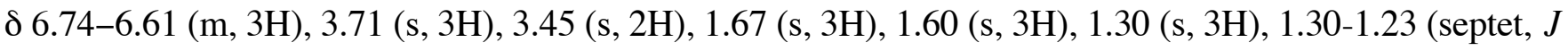
$=7.3 \mathrm{~Hz}, 3 \mathrm{H}), 1.06(\mathrm{~d}, J=7.2 \mathrm{~Hz}, 18 \mathrm{H}) ;{ }^{13} \mathrm{C} \mathrm{NMR}\left(\mathrm{CDCl}_{3}, 75 \mathrm{MHz}\right) \delta 169.6,149.9,144.4,125.8,122.0$, $120.1,110.3$, 104.7, 54.6, 50.5, 39.0, 29.9, 28.0, 24.2, 18.1, 14.2; HRMS(EI) $m / z$ calcd for $\mathrm{C}_{24} \mathrm{H}_{38} \mathrm{O}_{6} \mathrm{Si}$ $\left(\mathrm{M}^{+}-\mathrm{CH}_{3}\right)$ : 435.2203. Found: 435.2218. 
5-Methoxy-2-methyl-4-[(1,1,1-triisopropylsilyl)oxy]-1-indanone (101): Prepared from 100 in $77 \%$ yield using Procedure D in $40 \mathrm{~min}$. Purified by flash chromatography (10:1 hexanes:EtOAc) to provide a pale yellow oil. ${ }^{1} \mathrm{H}$ NMR $\left(\mathrm{CDCl}_{3}, 300 \mathrm{MHz}\right) \delta 7.36(\mathrm{~d}, J=8.3 \mathrm{~Hz}, 1 \mathrm{H}), 6.88(\mathrm{~d}, J=8.3 \mathrm{~Hz}, 1 \mathrm{H})$, $3.85(\mathrm{~s}, 2 \mathrm{H}, \mathrm{s}), 3.32(\mathrm{dd}, J=16.8,7.6 \mathrm{~Hz}, 1 \mathrm{H}), 2.64-2.59(\mathrm{~m}, 2 \mathrm{H}), 1.25$ (septet, $J=6.8 \mathrm{~Hz}, 6 \mathrm{H}), 1.07(\mathrm{~d}, J$ $=7.1 \mathrm{~Hz}, 18 \mathrm{H}) ;{ }^{13} \mathrm{C} \mathrm{NMR}\left(\mathrm{CDCl}_{3}, 75 \mathrm{MHz}\right) \delta 208.3,155.0,144.3,142.1,130.4,117.4,111.5,55.3,42.2$, 32.2, 18.0, 16.6, 13.7; HRMS(EI) $m / z$ calcd for $\mathrm{C}_{20} \mathrm{H}_{32} \mathrm{O}_{3} \mathrm{Si}\left(\mathrm{M}^{+}\right)$: 348.2121. Found: 348.2129.

5,5-Dibenzyl-2,2-dimethyl-1,3-dioxane-4,6-dione ${ }^{26}$ (102): Prepared according to Procedure B from Meldrum's acid and benzyl bromide. The reaction mixture in DMF was poured into $\mathrm{H}_{2} \mathrm{O}$ and stirred vigorously for 10 minutes, and the resulting white solid was filtered and dried under high vacuum to provide a white powder in $90 \%$ yield. M.p. $225-227{ }^{\circ} \mathrm{C}\left(\mathrm{H}_{2} \mathrm{O}\right) ;{ }^{1} \mathrm{H}$ NMR $\left(\mathrm{CDCl}_{3}, 300 \mathrm{MHz}\right) \delta 7.25-7.17$ (m, 10H), $3.42(\mathrm{~s}, 4 \mathrm{H}), 0.60(\mathrm{~s}, 6 \mathrm{H}) ;{ }^{13} \mathrm{C} \mathrm{NMR}\left(\mathrm{CDCl}_{3}, 75 \mathrm{MHz}\right) \delta 168.1,134.8,130.1,128.7,127.7$, 105.8, 60.0, 44.8, 28.5; Anal. Calcd for $\mathrm{C}_{20} \mathrm{H}_{20} \mathrm{O}_{4}$ : C, 74.06; H, 6.21. Found: C, 73.97; H, 6.18.

2-Benzyl-1-indanone ${ }^{25}$ (103): Prepared from 102 in 66\% yield using Procedure D in 50 min. Using catalytic TfOH gave 71\% yield in the same time period. Purified by flash chromatography (10:1 hexanes:EtOAc) to provide a clear colorless oil. ${ }^{1} \mathrm{H}$ NMR $\left(\mathrm{CDCl}_{3}, 300 \mathrm{MHz}\right) \delta 7.77(\mathrm{~d}, J=7.6 \mathrm{~Hz}, 1 \mathrm{H})$, $7.55(\mathrm{t}, J=7.4 \mathrm{~Hz}, 1 \mathrm{H}), 7.38-7.20(\mathrm{~m}, 7 \mathrm{H}), 3.39(\mathrm{dd}, J=4.2,3.9 \mathrm{~Hz}, 1 \mathrm{H}), 3.15(\mathrm{dd}, J=17.0,7.7 \mathrm{~Hz}, 1 \mathrm{H})$, 3.10-2.90 (m, 1H), $2.84(\mathrm{dd}, J=17.0,3.9 \mathrm{~Hz}, 1 \mathrm{H}), 2.65(\mathrm{dd}, J=13.9,10.4 \mathrm{~Hz}, 1 \mathrm{H}) .{ }^{13} \mathrm{C} \mathrm{NMR}\left(\mathrm{CDCl}_{3}, 75\right.$ MHz) $\delta 207.7,153.5,139.6,136.5,134.7,128.8,128.4,127.3,126.5,126.3,123.9,48.8,36.9,32.1$; HRMS(EI) $m / z$ calcd for $\mathrm{C}_{16} \mathrm{H}_{14} \mathrm{O}\left(\mathrm{M}^{+}\right)$: 222.1045. Found: 222.1052 .

5,5-Di(4-fluorobenzyl)-2,2-dimethyl-1,3-dioxane-4,6-dione (104): The dialkylation of Meldrum's acid with 4-fluorobenzyl bromide was performed according to Procedure B. The product was precipitated by addition of water, then filtered and dried under high vacuum. Recrystallization from $\mathrm{MeOH}$ provided a white solid in $74 \%$ yield. M.p. $144-145^{\circ} \mathrm{C}(\mathrm{MeOH}) ;{ }^{1} \mathrm{H}$ NMR $\left(\mathrm{CDCl}_{3}, 300 \mathrm{MHz}\right) \delta$ 7.16-7.11 (m, 4H), 6.96-6.90 (m, 4H), $3.67(\mathrm{~s}, 4 \mathrm{H}), 0.68(\mathrm{~s}, 6 \mathrm{H}) ;{ }^{13} \mathrm{C} \mathrm{NMR}\left(\mathrm{CDCl}_{3}, 75 \mathrm{MHz}\right) \delta 168.0,164.0,160.7,131.8$, 131.7, 130.5, 115.8, 105.9, 60.0, 43.9, 28.7; $\mathrm{HRMS}(\mathrm{EI}) \mathrm{m} / z$ calcd for $\mathrm{C}_{20} \mathrm{H}_{18} \mathrm{~F}_{2} \mathrm{O}_{4}\left(\mathrm{M}^{+}\right): 360.1173$. Found: 360.1166.

2,2-Dimethyl-5,5-di(4-nitrobenzyl)-1,3-dioxane-4,6-dione ${ }^{26}$ (106): The dialkylation of Meldrum's acid with 4-nitrobenzyl bromide was performed according to Procedure B. The product was precipitated by addition of water, then filtered and dried under high vacuum. Recrystallization from $\mathrm{MeOH}$ provided a 
white solid in $89 \%$ yield. M.p. $237-238^{\circ} \mathrm{C}(\mathrm{MeOH})$. Lit. $258-259^{\circ} \mathrm{C} ;{ }^{1} \mathrm{H}$ NMR $\left(\mathrm{CDCl}_{3}, 300 \mathrm{MHz}\right) \delta 8.16$ $(\mathrm{d}, J=8.6 \mathrm{~Hz}, 4 \mathrm{H}), 7.38(\mathrm{~d}, J=8.6 \mathrm{~Hz}, 4 \mathrm{H}), 3.56(\mathrm{~s}, 4 \mathrm{H}), 0.72(\mathrm{~s}, 6 \mathrm{H}) ;{ }^{13} \mathrm{C} \mathrm{NMR}\left(\mathrm{CDCl}_{3}, 75 \mathrm{MHz}\right) \delta$ 167.3, 147.7, 141.6, 131.3, 130.9, 124.0, 123.8, 106.3, 59.0, 44.4, 29.0.

5,5-Di(3-fluorobenzyl)-2,2-dimethyl-1,3-dioxane-4,6-dione (108): Dialkylation of Meldrum's acid according to Procedure B with 3-fluorobenzyl bromide to provide a white solid in a yield of $58 \%$ after recrystallization from $\mathrm{MeOH}$. M.p. $137-138{ }^{\circ} \mathrm{C}(\mathrm{MeOH}) ;{ }^{1} \mathrm{H}$ NMR $\left(\mathrm{CDCl}_{3}, 300 \mathrm{MHz}\right) \delta$ 7.27-7.19 (m, 2H), 7.00-6.88 (m, 6H), $3.40(\mathrm{~s}, 4 \mathrm{H}), 0.71(\mathrm{~s}, 6 \mathrm{H}) ;{ }^{13} \mathrm{C} \mathrm{NMR}\left(\mathrm{CDCl}_{3}, 75 \mathrm{MHz}\right) \delta$ 167.8, 164.4, 161.1, 136.9, 130.3, 125.9, $117.2(\mathrm{~d}, J=22.5 \mathrm{~Hz}), 114.9$ (d, $J=15.0 \mathrm{~Hz}), 106.0,59.5,44.5,28.7$; HRMS(EI) $\mathrm{m} / z$ calcd for $\mathrm{C}_{20} \mathrm{H}_{18} \mathrm{~F}_{2} \mathrm{O}_{4}\left(\mathrm{M}^{+}\right)$: 360.1173 . Found: 360.1177 .

5-Fluoro-2-(3-fluorobenzyl)-1-indanone (109): Prepared from 108 in 93\% yield as a 13:1 mixture of para 109:ortho 110 products using Procedure D in $90 \mathrm{~min}$. The mixture was separated by flash chromatography (13:1 hexanes:EtOAc) to provide clear colorless oils. ${ }^{1} \mathrm{H}$ NMR $\left(\mathrm{CDCl}_{3}, 300 \mathrm{MHz}\right)$

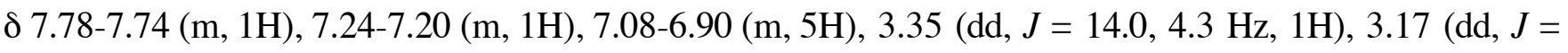
$17.2,7.8 \mathrm{~Hz}, 1 \mathrm{H}), 3.05-2.90(\mathrm{~m}, 1 \mathrm{H}), 2.80(\mathrm{dd}, J=17.1,4.2 \mathrm{~Hz}, 1 \mathrm{H}), 2.68(\mathrm{dd}, J=13.9,10.1 \mathrm{~Hz}, 1 \mathrm{H}) ;{ }^{13} \mathrm{C}$ NMR $\left(\mathrm{CDCl}_{3}, 75 \mathrm{MHz}\right) \delta 205.4,166.8(\mathrm{~d}, J=336.2 \mathrm{~Hz}), 163.4(\mathrm{~d}, J=325.7 \mathrm{~Hz}), 156.3(\mathrm{~d}, J=10.0 \mathrm{~Hz})$, 141.9 (d, $J=7.1 \mathrm{~Hz}), 132.8(\mathrm{~d}, J=1.7 \mathrm{~Hz}), 130.0(\mathrm{~d}, J=8.4 \mathrm{~Hz}), 126.3(\mathrm{~d}, J=10.5 \mathrm{~Hz}), 124.5,115.8$ (d, $J=23.7 \mathrm{~Hz}), 115.7$ (d, $J=20.9 \mathrm{~Hz}), 113.4(\mathrm{~d}, J=20.9 \mathrm{~Hz}), 113.2$ (d, $J=22.1 \mathrm{~Hz}), 48.8,36.6,32.0$; HRMS(EI) $\mathrm{m} / z$ calcd for $\mathrm{C}_{16} \mathrm{H}_{12} \mathrm{~F}_{2} \mathrm{O}(\mathrm{M}+)$ : 258.0856. Found: 258.0851.

7-Fluoro-2-(3-fluorobenzyl)-1-indanone (110): ${ }^{1} \mathrm{H}$ NMR $\left(\mathrm{CDCl}_{3}, 300 \mathrm{MHz}\right) \delta$ 7.80-7.75 (m, 1H), 7.28-7.24 (m, 1H), 7.09-6.92 (m, 5H), $3.36(\mathrm{dd}, J=14.0,4.3 \mathrm{~Hz}, 1 \mathrm{H}), 3.18(\mathrm{dd}, J=17.3,7.8 \mathrm{~Hz}, 1 \mathrm{H})$, 3.20-2.99 (m, 1H), $2.84(\mathrm{dd}, J=17.3,3.9 \mathrm{~Hz}, 1 \mathrm{H}), 2.70(\mathrm{dd}, J=14.0,10.1 \mathrm{~Hz}, 1 \mathrm{H}) ;{ }^{13} \mathrm{C}$ NMR $\left(\mathrm{CDCl}_{3}, 75\right.$

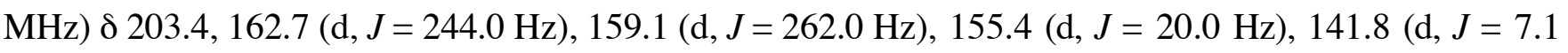
Hz), $136.8(\mathrm{~d}, J=8.3 \mathrm{~Hz}), 130.0(\mathrm{~d}, J=8.3 \mathrm{~Hz}), 124.6,122.4,115.7$ (d, $J=20.9 \mathrm{~Hz}), 114.4(\mathrm{~d}, J=19.1$ $\mathrm{Hz}), 113.4(\mathrm{~d}, J=20.9 \mathrm{~Hz}), 49.1,36.5,32.0 ; \mathrm{HRMS}(\mathrm{EI}) \mathrm{m} / \mathrm{z}$ calcd for $\mathrm{C}_{16} \mathrm{H}_{12} \mathrm{~F}_{2} \mathrm{O}\left(\mathrm{M}^{+}\right)$: 258.0856. Found: 258.0858 .

\section{5-[(1-Benzyl-3-piperidyl)methyl]-5-(3,4-dimethoxybenzyl)-2,2-dimethyl-1,3-dioxane-4,6-}

dione (111): Reductive alkyation of Meldrum's acid according to Procedure A using N-benzyl-4formylpiperidine (prepared by Swern oxidation of (1-benzylpiperidin-4-yl)methanol) ${ }^{27}$ provided the monosubstituted derivative in $47 \%$ yield after flash chromatography $\left(50: 1 \mathrm{CH}_{2} \mathrm{Cl}_{2}: \mathrm{MeOH}\right)$. This yellow oil 
was alkylated with 3,4-dimethoxybenzyl bromide ${ }^{20}$ according to Procedure $\mathrm{B}$, and the target material was isolated as a pale yellow solid in 44\% yield following flash chromatography (5:2 EtOAc:hexanes) and recrystallization from $\mathrm{MeOH}$. M.p. $120-121^{\circ} \mathrm{C}(\mathrm{MeOH}) ;{ }^{1} \mathrm{H} \mathrm{NMR}\left(\mathrm{CDCl}_{3}, 300 \mathrm{MHz}\right) \delta 7.25-7.17$ (m, 5H), 6.70-6.64 (m, 3H), 3.78 (s, 3H), 3.77 (s, 3H), 3.39 (s, 2H), 3.17 (s, 2H), 2.80-2.76 (m, 2H), 2.10-2.07 $(\mathrm{m}, 2 \mathrm{H}), 1.84-1.80(\mathrm{~m}, 2 \mathrm{H}), 1.60-1.52(\mathrm{~m}, 2 \mathrm{H}), 1.52(\mathrm{~s}, 3 \mathrm{H}), 1.35-1.20(\mathrm{~m}, 3 \mathrm{H}), 0.65(\mathrm{~s}, 3 \mathrm{H}) ;{ }^{13} \mathrm{C}$ NMR $\left(\mathrm{CDCl}_{3}, 75 \mathrm{MHz}\right) \delta 169.0,148.9,148.5,138.2,128.9,128.0,127.5,126.8,122.5,113.2,111.2,105.8$, 63.2, 56.2, 55.8, 55.7, 53.3, 47.9, 44.4, 33.2, 32.7, 29.1, 29.1; HRMS(EI) $m / z$ calcd for $\mathrm{C}_{28} \mathrm{H}_{35} \mathrm{NO}_{6}\left(\mathrm{M}^{+}\right)$: 481.2464. Found: 481.2458.

2-[(1-Benzyl-4-piperidyl)methyl]-5,6-dimethoxy-1-indanone (donepezil) (112): Prepared from 111 in $61 \%$ yield using Procedure D with $\mathrm{TfOH}$ (1.2 eq) in $30 \mathrm{~min}$. After removal of nitromethane, the crude brown oil was dissolved in $\mathrm{CH}_{2} \mathrm{Cl}_{2}$ and washed with saturated $\mathrm{NaHCO}_{3}$ (2 times) then the organic layer washed with brine, then dried over $\mathrm{MgSO}_{4}$ and filtered. Concentration, followed by flash chromatography $\left(20: 1 \mathrm{CH}_{2} \mathrm{Cl}_{2}: \mathrm{MeOH}\right)$ provided a pale yellow oil. ${ }^{1} \mathrm{H} \mathrm{NMR}\left(\mathrm{CDCl}_{3}, 300 \mathrm{MHz}\right) \delta 7.30$ $7.14(\mathrm{~m}, 5 \mathrm{H}), 7.14(\mathrm{~s}, 3 \mathrm{H}), 6.83(\mathrm{~s}, 1 \mathrm{H}), 3.93(\mathrm{~s}, 3 \mathrm{H}), 3.88(\mathrm{~s}, 3 \mathrm{H}), 3.51(\mathrm{~s}, 2 \mathrm{H}), 3.21(\mathrm{dd}, J=17.5,8.1 \mathrm{~Hz}$, $1 \mathrm{H}), 2.92-2.87(\mathrm{~m}, 2 \mathrm{H}), 2.70-2.63(\mathrm{~m}, 2 \mathrm{H}), 2.02-1.80(\mathrm{~m}, 3 \mathrm{H}), 1.75-1.60(\mathrm{~m}, 2 \mathrm{H}), 1.55-1.20(\mathrm{~m}, 4 \mathrm{H}) ;{ }^{13} \mathrm{C}$ NMR $\left(\mathrm{CDCl}_{3}, 75 \mathrm{MHz}\right) \delta 207.7,155.4,149.4,148.7,138.1,129.3,128.1,127.0,107.3,104.4,63.3,56.2$, 56.1, 53.7, 45.4, 38.7, 34.3, 33.4, 32.8, 31.7; $\mathrm{HRMS}(\mathrm{EI}) \mathrm{m} / \mathrm{z}$ calcd for $\mathrm{C}_{24} \mathrm{H}_{29} \mathrm{NO}_{3}\left(\mathrm{M}^{+}\right)$: 379.2147. Found: 379.2142 .

5-(3,4-Dimethoxybenzyl)-2,2-dimethyl-5-(4-pyridylmethyl)-1,3-dioxane-4,6-dione (113): Alkylation of 27 according to Procedure B with (4-bromomethyl)pyridine hydrobromide provided a 74\% yield of an off-white solid after recrystallization from MeOH. M.p. $139-141{ }^{\circ} \mathrm{C}(\mathrm{MeOH}) ;{ }^{1} \mathrm{H}$ NMR $\left(\mathrm{CDCl}_{3}, 300 \mathrm{MHz}\right) \delta 8.51(\mathrm{~d}, J=5.8 \mathrm{~Hz}, 2 \mathrm{H}), 7.10(\mathrm{~d}, J=5.8 \mathrm{~Hz}, 2 \mathrm{H}), 6.74-6.68(\mathrm{~m}, 3 \mathrm{H}), 3.82(\mathrm{~s}, 3 \mathrm{H})$, $3.80(\mathrm{~s}, 3 \mathrm{H}), 3.39(\mathrm{~s}, 2 \mathrm{H}), 3.38(\mathrm{~s}, 2 \mathrm{H}), 0.74(\mathrm{~s}, 3 \mathrm{H}), 0.68(\mathrm{~s}, 3 \mathrm{H}) ;{ }^{13} \mathrm{C} \mathrm{NMR}\left(\mathrm{CDCl}_{3}, 75 \mathrm{MHz}\right) \delta 167.5$, $149.9,148.6,148.4,143.2,128.4,124.7,122.0,112.7,110.9,105.9,59.1,55.5,44.3,43.6,28.5,28.4$; HRMS(EI) $m / z$ calcd for $\mathrm{C}_{21} \mathrm{H}_{23} \mathrm{NO}_{6}\left(\mathrm{M}^{+}\right)$: 385.1525. Found: 385.1522 .

5,6-Dimethoxy-2-(4-pyridylmethyl)-1-indanone ${ }^{28}$ (114): Prepared from 113 using Procedure D, using TfOH (1.2 eq) in $45 \mathrm{~min}$. After removal of nitromethane, the crude brown oil was dissolved in $\mathrm{CH}_{2} \mathrm{Cl}_{2}$ and washed with saturated $\mathrm{NaHCO}_{3}$ (2 times), brine, then dried over $\mathrm{MgSO}_{4}$ and filtered. Concentration, followed by flash chromatography $\left(20: 1 \mathrm{CH}_{2} \mathrm{Cl}_{2}: \mathrm{MeOH}\right)$ provided a pale yellow solid in 
$77 \%$ yield. The use of TMSOTf (1.2 eq) instead of TfOH provided a $62 \%$ yield. Recrystallization from $\mathrm{MeOH}$ afforded a white powder. M.p. $190-191{ }^{\circ} \mathrm{C}(\mathrm{MeOH})$. Lit. 190-191 ${ }^{\circ} \mathrm{C} ;{ }^{1} \mathrm{H}$ NMR $\left(\mathrm{CDCl}_{3}, 300\right.$ MHz) $\delta 8.49-8.48(\mathrm{~m}, 2 \mathrm{H}), 7.17-7.14(\mathrm{~m}, 3 \mathrm{H}), 6.79(\mathrm{~s}, 1 \mathrm{H}), 3.92(\mathrm{~s}, 3 \mathrm{H}), 3.81(\mathrm{~s}, 3 \mathrm{H}), 3.32(\mathrm{dd}, J=14.1$ $\mathrm{Hz}, 4.1 \mathrm{~Hz}, 1 \mathrm{H}), 3.09$ (dd, $J=16.7 \mathrm{~Hz}, 7.5 \mathrm{~Hz}, 1 \mathrm{H}), 3.00-2.90(\mathrm{~m}, 1 \mathrm{H}), 2.71-2.63(\mathrm{~m}, 2 \mathrm{H})$; ${ }^{13} \mathrm{C} \mathrm{NMR}$ $\left(\mathrm{CDCl}_{3}, 75 \mathrm{MHz}\right) \delta 205.1,155.4,149.5,149.3,148.5,148.3,128.6,124.0,107.1,104.0,55.9,55.7,47.6$, 36.1, 31.5; $\mathrm{HRMS}(\mathrm{EI}) \mathrm{m} / \mathrm{z}$ calcd for $\mathrm{C}_{17} \mathrm{H}_{17} \mathrm{NO}_{3}\left(\mathrm{M}^{+}\right)$: 283.1208. Found: 283.1205.

5-(3,4-Dimethoxyphenethyl)-2,2-dimethyl-1,3-dioxane-4,6-dione (115): Condensation and reduction of Meldrum's acid and 3,4-dimethoxyphenylacetic acid according to the procedure of Tsukamoto $^{29}$ provided a white powder in $79 \%$ yield after recrystallization from $\mathrm{MeOH}$. M.p. $138-139.5^{\circ} \mathrm{C}$ $(\mathrm{MeOH}) ;{ }^{1} \mathrm{H} \mathrm{NMR}\left(\mathrm{CDCl}_{3}, 300 \mathrm{MHz}\right) \delta$ 6.77-6.73 (m, 3H), $3.85(\mathrm{~s}, 3 \mathrm{H}), 3.84(\mathrm{~s}, 3 \mathrm{H}), 3.46(\mathrm{t}, J=5.3 \mathrm{~Hz}$, $1 \mathrm{H}), 2.81-2.76(\mathrm{~m}, 2 \mathrm{H}), 2.41-2.36(\mathrm{~m}, 2 \mathrm{H}), 1.74(\mathrm{~s}, 3 \mathrm{H}), 1.72(\mathrm{~s}, 3 \mathrm{H}) ;{ }^{13} \mathrm{C} \mathrm{NMR}\left(\mathrm{CDCl}_{3}, 75 \mathrm{MHz}\right) \delta$ $165.3,148.8,147.3,132.7,120.4,111.6,111.1,104.7,55.7,55.6,44.8,31.8,28.3,27.9,26.4$; HRMS(EI) $m / z$ calcd for $\mathrm{C}_{16} \mathrm{H}_{20} \mathrm{O}_{6}\left(\mathrm{M}^{+}\right): 308.1260$. Found: 308.1257 .

6,7-Dimethoxy-1,2,3,4-tetrahydro-1-naphthalenone ${ }^{30}$ (116): Prepared from 115 in 82\% yield using Procedure D in 45 min. Purified by flash chromatography (1:1 hexanes:EtOAc) to provide a white solid. M.p. 96-98 ${ }^{\circ} \mathrm{C}\left(\mathrm{CH}_{2} \mathrm{Cl}_{2}\right)$. Lit. 98-99 ${ }^{\circ} \mathrm{C} ;{ }^{1} \mathrm{H} \mathrm{NMR}\left(\mathrm{CDCl}_{3}, 300 \mathrm{MHz}\right) \delta 7.48(\mathrm{~s}, 1 \mathrm{H}), 6.64$ (s, 1H), 3.90 (s, $3 \mathrm{H}), 3.88(\mathrm{~s}, 3 \mathrm{H}), 2.88-2.84(\mathrm{~m}, 2 \mathrm{H}), 2.58-2.54(\mathrm{~m}, 2 \mathrm{H}), 2.10-2.07$ (m, 2H) ${ }^{13} \mathrm{C} \mathrm{NMR}\left(\mathrm{CDCl}_{3}, 75 \mathrm{MHz}\right)$ $\delta 197.1,153.3,147.8,139.2,128.7,110.1,108.3,55.9,38.4,29.3,23.5 ; \mathrm{HRMS}(\mathrm{EI}) \mathrm{m} / \mathrm{z}$ calcd for $\mathrm{C}_{12} \mathrm{H}_{14} \mathrm{O}_{3}: 206.0943$. Found: 206.0950 .

5-(3,4-Dimethoxyphenethyl)-2,2,5-trimethyl-1,3-dioxane-4,6-dione (117): Alkylation of 115 according to Procedure B using iodomethane, followed by recrystallization from $\mathrm{MeOH}$ provided an $82 \%$ yield of white powder. M.p. 81.5-83 ${ }^{\circ} \mathrm{C}(\mathrm{MeOH}) ;{ }^{1} \mathrm{H} \mathrm{NMR}\left(\mathrm{CDCl}_{3}, 300 \mathrm{MHz}\right) \delta$ 6.70-6.57 (m, 3H), 3.76 (s, 6H), $3.74(\mathrm{~s}, 3 \mathrm{H}), 2.43-2.38(\mathrm{~m}, 2 \mathrm{H}), 2.8-2.24(\mathrm{~m}, 2 \mathrm{H}), 1.67$ (br s, 6H), 1.57 (s, 3H); ${ }^{13} \mathrm{C} \mathrm{NMR}\left(\mathrm{CDCl}_{3}\right.$, $75 \mathrm{MHz}) \delta 170.0,148.7,147.4,132.1,120.0,111.4,111.1,104.7,55.6,55.6,49.2,42.8,31.2,29.5,28.2$, 24.0; $\mathrm{HRMS}(\mathrm{EI}) \mathrm{m} / z$ calcd for $\mathrm{C}_{17} \mathrm{H}_{22} \mathrm{O}_{6}\left(\mathrm{M}^{+}\right)$: 322.1416. Found: 322.1419 .

6,7-Dimethoxy-2-methyl-1,2,3,4-tetrahydro-1-naphthalenone ${ }^{31}$ (118): Prepared from 117 in $82 \%$ yield using Procedure D in 15 min. Purified by flash chromatography (3:1 hexanes:EtOAc) to provide a pale yellow solid. M.p. $120-121{ }^{\circ} \mathrm{C}\left(\mathrm{CH}_{2} \mathrm{Cl}_{2}\right)$. Lit. $129-130{ }^{\circ} \mathrm{C} ;{ }^{1} \mathrm{H} \mathrm{NMR}\left(\mathrm{CDCl}_{3}, 300 \mathrm{MHz}\right) \delta 7.49$ (s, $1 \mathrm{H}), 6.62(\mathrm{~s}, 1 \mathrm{H}), 3.90(\mathrm{~s}, 3 \mathrm{H}), 3.88(\mathrm{~s}, 3 \mathrm{H}), 3.00-2.80(\mathrm{~m}, 2 \mathrm{H}), 2.53-2.45(\mathrm{~m}, 1 \mathrm{H}), 2.18-2.12(\mathrm{~m}, 1 \mathrm{H})$, 
$1.90-1.75(\mathrm{~m}, 1 \mathrm{H}), 1.23(\mathrm{~d}, J=6.8 \mathrm{~Hz}, 3 \mathrm{H}) ;{ }^{13} \mathrm{C} \mathrm{NMR}\left(\mathrm{CDCl}_{3}, 75 \mathrm{MHz}\right) \delta 199.5,153.1,147.1,138.8$, 125.3, 109.9, 108.5, 55.8, 41.7, 31.5, 28.3, 15.4; HRMS(EI) $m / z$ calcd for $\mathrm{C}_{13} \mathrm{H}_{16} \mathrm{O}_{3}: 220.1099$. Found: 220.1107.

5-[3-(3,4-Dimethoxyphenyl)propyl]-2,2-dimethyl-1,3-dioxane-4,6-dione (119): Condensation and reduction of Meldrum's acid and 3,4-dimethoxyhydrocinnamic acid according to Tsukamoto ${ }^{29}$ provided a white solid in $68 \%$ yield after recrystallization from $\mathrm{MeOH}$. M.p. $113-114.5{ }^{\circ} \mathrm{C}(\mathrm{MeOH}) ;{ }^{1} \mathrm{H}$ $\operatorname{NMR}\left(\mathrm{CDCl}_{3}, 300 \mathrm{MHz}\right) \delta$ 6.76-6.67 (m, 3H), $3.83(\mathrm{~s}, 3 \mathrm{H}), 3.80(\mathrm{~s}, 3 \mathrm{H}), 3.48(\mathrm{t}, J=4.9 \mathrm{~Hz}, 1 \mathrm{H}), 2.60(\mathrm{t}$, $J=7.6 \mathrm{~Hz}, 2 \mathrm{H}), 2.14-2.07(\mathrm{~m}, 2 \mathrm{H}), 1.80-1.68(\mathrm{~m}, 2 \mathrm{H}), 1.69($ br s, $6 \mathrm{H}) ;{ }^{13} \mathrm{C} \mathrm{NMR}\left(\mathrm{CDCl}_{3}, 75 \mathrm{MHz}\right) \delta$ $165.4,148.7,147.1,134.0,120.1,111.4,111.1,104.7,55.8,55.4,45.9,35.2,28.3,27.8,26.6,26.0$; HRMS(EI) $m / z$ calcd for $\mathrm{C}_{17} \mathrm{H}_{22} \mathrm{O}_{6}\left(\mathrm{M}^{+}\right): 322.1416$. Found: 322.1407 .

2,3-Dimethoxy-6,7,8,9-tetrahydro-5H-benzo[a]cyclohepten-5-one ${ }^{32}$ (120): Prepared from 119 in 78\% yield using Procedure D in $45 \mathrm{~min}$. Purified by flash chromatography (1:1 hexanes:EtOAc) to provide a white solid. M.p. 61-62.5 ${ }^{\circ} \mathrm{C}\left(\mathrm{CH}_{2} \mathrm{Cl}_{2}\right)$. Lit. 63-64 ${ }^{\circ} \mathrm{C} ;{ }^{1} \mathrm{H}$ NMR $\left(\mathrm{CDCl}_{3}, 300 \mathrm{MHz}\right) \delta 7.35(\mathrm{~s}$, $1 \mathrm{H}), 6.65(\mathrm{~s}, 1 \mathrm{H}), 3.91(\mathrm{~s}, 3 \mathrm{H}), 3.88(\mathrm{~s}, 3 \mathrm{H}), 2.90-2.86(\mathrm{~m}, 2 \mathrm{H}), 2.73-2.69(\mathrm{~m}, 2 \mathrm{H}), 1.87-1.74(\mathrm{~m}, 4 \mathrm{H}) ;{ }^{13} \mathrm{C}$ $\operatorname{NMR}\left(\mathrm{CDCl}_{3}, 75 \mathrm{MHz}\right) \delta 203.7,152.0,147.3,136.6,130.6,112.2,111.1,55.8,40.6,32.4,25.0,20.5$; HRMS(EI) $m / z$ calcd for $\mathrm{C}_{13} \mathrm{H}_{16} \mathrm{O}_{3}: 220.1099$ Found: 220.1096 .

5-[3-(3,4-Dimethoxyphenyl)propyl]-2,2,5-trimethyl-1,3-dioxane-4,6-dione (121): Alkylation of 119 using Procedure B with iodomethane, followed by recrystallization from $\mathrm{MeOH}$ provided a 79\% yield of a white powder. M.p. 80-81 ${ }^{\circ} \mathrm{C}(\mathrm{MeOH}) ;{ }^{1} \mathrm{H}$ NMR $\left(\mathrm{CDCl}_{3}, 300 \mathrm{MHz}\right) \delta 6.77-6.63(\mathrm{~m}, 3 \mathrm{H}), 3.85(\mathrm{~s}$, $3 \mathrm{H}), 3.83(\mathrm{~s}, 3 \mathrm{H}), 2.52(\mathrm{t}, J=7.6 \mathrm{~Hz}, 2 \mathrm{H}), 2.06-2.00(\mathrm{~m}, 2 \mathrm{H}), 1.71(\mathrm{~s}, 3 \mathrm{H}), 1.70(\mathrm{~s}, 3 \mathrm{H}), 1.60(\mathrm{~s}, 3 \mathrm{H}), 1.60-$ $1.40(\mathrm{~m}, 2 \mathrm{H}) ;{ }^{13} \mathrm{C} \mathrm{NMR}\left(\mathrm{CDCl}_{3}, 75 \mathrm{MHz}\right) \delta$ 170.2, 148.6, 147.1, 133.3, 120.2, 111.2, 111.0, 104.7, 55.7, 55.6, 49.3, 40.5, 34.8, 29.6, 28.3, 27.0, 24.0; $\mathrm{HRMS}(\mathrm{EI}) \mathrm{m} / \mathrm{z}$ calcd for $\mathrm{C}_{18} \mathrm{H}_{24} \mathrm{O}_{6}\left(\mathrm{M}^{+}\right)$: 336.1573. Found: 336.1585.

2,3-Dimethoxy-6-methyl-6,7,8,9-tetrahydro-5H-benzo[a]cyclohepten-5-one (122): Prepared from 121 in $81 \%$ yield using Procedure D in 15 min. Purified by flash chromatography (1:1 hexanes:EtOAc) to provide a pale yellow solid. M.p. $80-81{ }^{\circ} \mathrm{C}\left(\right.$ from $\left.\mathrm{CH}_{2} \mathrm{Cl}_{2}\right) ;{ }^{1} \mathrm{H} \mathrm{NMR}\left(\mathrm{CDCl}_{3}, 300 \mathrm{MHz}\right) \delta 7.33(\mathrm{~s}, 1 \mathrm{H})$, $6.65(\mathrm{~s}, 1 \mathrm{H}), 3.91$ (s, 3H), 3.89 (s, 3H), 3.05-2.84 (m, 3H), 2.10-1.90 (m, 1H), 1.89-1.82 (m, 1H), 1.70$1.50(\mathrm{~m}, 2 \mathrm{H}), 1.20(\mathrm{~d}, J=6.6 \mathrm{~Hz}, 3 \mathrm{H}) .{ }^{13} \mathrm{C} \mathrm{NMR}\left(\mathrm{CDCl}_{3}, 75 \mathrm{MHz}\right) \delta 205.0,151.2,147.0,137.2,131.3$, 
112.3, 111.1, 55.8, 43.5, 43.4, 31.2, 25.5, 16.4; $\mathrm{HRMS}(\mathrm{EI}) \mathrm{m} / \mathrm{z}$ calcd for $\mathrm{C}_{14} \mathrm{H}_{18} \mathrm{O}_{3}$ : 234.1256. Found: 234.1251.

5-Benzyl-2,2-dimethyl-5-phenethyl-1,3-dioxane-4,6-dione (123): 2,2-Dimethyl-5-phenethyl-1,3dioxane-4,6-dione ${ }^{29}$ was alkylated with benzyl bromide according to Procedure B. Purification by recrystallization from $\mathrm{MeOH}$ provided a crystalline solid in 22\% yield (3 steps from Meldrum's acid); M.p. 117.0-118.0 ${ }^{\circ} \mathrm{C}(\mathrm{MeOH}) ;{ }^{1} \mathrm{H}$ NMR $\left(\mathrm{CDCl}_{3}, 300 \mathrm{MHz}\right) \delta$ 7.30-7.15 (m, 10H), 3.33 (s, 2H), 2.59$2.38(\mathrm{~m}, 4 \mathrm{H}), 1.58(\mathrm{~s}, 3 \mathrm{H}), 0.65(\mathrm{~s}, 3 \mathrm{H}) ;{ }^{13} \mathrm{C} \mathrm{NMR}\left(\mathrm{CDCl}_{3}, 75 \mathrm{MHz}\right) \delta 168.8,139.6,135.3,130.3,128.9$, 128.6, 128.3, 127.8, 126.5, 106.0, 57.6, 43.8, 43.1, 31.8, 29.5, 28.8; HRMS(EI) $m / z$ calcd for $\mathrm{C}_{18} \mathrm{H}_{16} \mathrm{O}_{3}$ $\left(\mathrm{M}^{+}-\mathrm{C}_{3} \mathrm{H}_{6} \mathrm{O}\right): 280.1099$. Found: 280.1088.

2-Benzyl-1,2,3,4-tetrahydro-1-naphthalenone ${ }^{33}$ (124): Obtained as the exclusive isomer in $75 \%$ yield from 123 using Procedure $\mathrm{D}$ in $45 \mathrm{~min}$. Purified by flash chromatography $\left(1: 1 \mathrm{CH}_{2} \mathrm{Cl}_{2}\right.$ :hexanes $)$ to afford a fine white powder. M.p. $50-51{ }^{\circ} \mathrm{C}\left(\mathrm{CH}_{2} \mathrm{Cl}_{2}\right.$ /hexanes). Lit. $53-54{ }^{\circ} \mathrm{C}$ (petroleum ether); ${ }^{1} \mathrm{H}$ NMR $\left(\mathrm{CDCl}_{3}, 300 \mathrm{MHz}\right) \delta 8.05(\mathrm{~d}, J=7.8 \mathrm{~Hz}, 1 \mathrm{H}), 7.45(\mathrm{dt}, J=7.1,1.2 \mathrm{~Hz}, 1 \mathrm{H}), 7.32-7.19(\mathrm{~m}, 7 \mathrm{H}), 3.48(\mathrm{dd}, J$ $=13.4,3.7 \mathrm{~Hz}, 1 \mathrm{H}), 2.94-2.89(\mathrm{~m}, 2 \mathrm{H}), 2.74-2.59(\mathrm{~m}, 1 \mathrm{H}), 2.62(\mathrm{dd}, J=13.4,9.6 \mathrm{~Hz}, 1 \mathrm{H}), 2.09(\mathrm{dq}, J=$ 13.4, $4.4 \mathrm{~Hz}, 1 \mathrm{H}), 1.84-1.70(\mathrm{~m}, 1 \mathrm{H}) ;{ }^{13} \mathrm{C} \mathrm{NMR}\left(\mathrm{CDCl}_{3}, 75 \mathrm{MHz}\right) \delta 199.4,144.0,140.0,133.3,132.5$, 129.3 $128.7,128.4,127.5,126.6,126.1,49.5,35.7,28.6,27.6$; $\mathrm{HRMS}(\mathrm{EI}) \mathrm{m} / z$ calcd for $\mathrm{C}_{17} \mathrm{H}_{16} \mathrm{O}\left(\mathrm{M}^{+}\right)$: 236.1201. Found: 236.1200.

5-Benzyl-2,2-dimethyl-5-(3-phenylpropyl)-1,3-dioxane-4,6-dione (125): Meldrum's acid was coupled with cinnamic acid using the method of Tsukamoto. $^{29}$ The resulting intermediate was hydrogenated over $5 \% \mathrm{Pd} / \mathrm{C}$, then alkylated with benzyl bromide according to Procedure $\mathrm{B}$, and purified by recrystallization from $\mathrm{MeOH}$ to afford a crystalline solid in $25 \%$ yield (3 steps); M.p. $126-127{ }^{\circ} \mathrm{C}$ $(\mathrm{MeOH}) ;{ }^{1} \mathrm{H}$ NMR $\left(\mathrm{CDCl}_{3}, 300 \mathrm{MHz}\right) \delta$ 7.29-7.11 (m, 10H), $3.29(\mathrm{~s}, 2 \mathrm{H}), 2.61(\mathrm{t}, J=7.6 \mathrm{~Hz}, 2 \mathrm{H}), 2.20-$ $2.15(\mathrm{~m}, 2 \mathrm{H}), 1.65-1.54(\mathrm{~m}, 2 \mathrm{H}), 1.51(\mathrm{~s}, 3 \mathrm{H}), 0.60(\mathrm{~s}, 3 \mathrm{H}) ;{ }^{13} \mathrm{C} \mathrm{NMR}\left(\mathrm{CDCl}_{3}, 75 \mathrm{MHz}\right) \delta 168.9,140.7$, $130.3,128.9,128.5,128.3,127.8,126.1,105.9,57.6,43.8,40.8,35.5,29.5,28.9,27.0 ; \mathrm{HRMS}(\mathrm{EI}) \mathrm{m} / z$ calcd for $\mathrm{C}_{19} \mathrm{H}_{18} \mathrm{O}_{3}\left(\mathrm{M}^{+}-\mathrm{C}_{3} \mathrm{H}_{6} \mathrm{O}\right):$ 294.1256. Found: 294.1249.

6-Benzyl-6,7,8,9-tetrahydro-5H-benzo[a]cyclohepten-5-one (126): Obtained as the exclusive isomer in 77\% yield from 125 using Procedure D in 45 min. Purified by flash chromatography (9:1 pentane:Et $\left.{ }_{2} \mathrm{O}\right)$ to afford a clear, colorless oil. ${ }^{1} \mathrm{H} \mathrm{NMR}\left(\mathrm{CDCl}_{3}, 300 \mathrm{MHz}\right) \delta 7.73(\mathrm{~d}, J=7.6 \mathrm{~Hz}, 1 \mathrm{H}), 7.56$ $(\mathrm{t}, J=7.2 \mathrm{~Hz}, 1 \mathrm{H}), 7.42(\mathrm{~d}, J=7.6 \mathrm{~Hz}, 1 \mathrm{H}), 7.34(\mathrm{t}, J=7.4 \mathrm{~Hz}, 1 \mathrm{H}), 7.29-7.01(\mathrm{~m}, 5 \mathrm{H}), 3.30(\mathrm{dd}, J=17.2$, 
$7.9 \mathrm{~Hz}, 1 \mathrm{H}), 2.78(\mathrm{dd}, J=17.2,3.9 \mathrm{~Hz}, 1 \mathrm{H}), 2.71-2.62(\mathrm{~m}, 3 \mathrm{H}), 2.05-1.93(\mathrm{~m}, 1 \mathrm{H}), 1.74$ (quint., $J=7.8$ $\mathrm{Hz}, 2 \mathrm{H}), 1.55-1.45(\mathrm{~m}, 1 \mathrm{H}) ;{ }^{13} \mathrm{C} \mathrm{NMR}\left(\mathrm{CDCl}_{3}, 75 \mathrm{MHz}\right) \delta 208.8,153.7,142.1,136.8,134.7,128.4,128.3$, 127.3, 126.5, 125.8, 123.9, 47.3, 36.0, 32.8, 31.1, 29.3; HRMS(EI) $m / z$ calcd for $\mathrm{C}_{18} \mathrm{H}_{18} \mathrm{O}\left(\mathrm{M}^{+}\right): 250.1358$. Found: 250.1358 .

\section{2,2-Dimethyl-5-phenethyl-5-(3-phenylpropyl)-1,3-dioxane-4,6-dione (127): 2,2-Dimethyl-5-} phenethyl-1,3-dioxane-4,6-dione ${ }^{29}$ was alkylated with cinnamyl chloride according to Procedure B, and the resulting intermediate hydrogenated over $5 \% \mathrm{Pd} / \mathrm{C}$ and recrystallized from $\mathrm{MeOH}$ to provide a crystalline solid in $72 \%$ yield (2 steps); M.p. 94.5-95.5 ${ }^{\circ} \mathrm{C}(\mathrm{MeOH}) ;{ }^{1} \mathrm{H}$ NMR $\left(\mathrm{CDCl}_{3}, 300 \mathrm{MHz}\right) \delta$ 7.28-7.09 (m, $10 \mathrm{H}), 2.62-2.53(\mathrm{~m}, 4 \mathrm{H}), 2.28-2.03(\mathrm{~m}, 4 \mathrm{H}), 1.74(\mathrm{~s}, 3 \mathrm{H}), 1.70(\mathrm{~s}, 3 \mathrm{H}), 1.70-1.56(\mathrm{~m}, 2 \mathrm{H}) ;{ }^{13} \mathrm{C}$ NMR $\left(\mathrm{CDCl}_{3}, 75 \mathrm{MHz}\right) \delta 169.2,128.6,128.5,128.3,126.1,105.7,54.5,29.8,27.1 ; \mathrm{HRMS}(\mathrm{EI}) \mathrm{m} / z$ calcd for $\mathrm{C}_{20} \mathrm{H}_{20} \mathrm{O}_{3}\left(\mathrm{M}^{+}-\mathrm{C}_{3} \mathrm{H}_{6} \mathrm{O}\right): 308.1412$. Found: 308.1407.

2-(3-Phenylpropyl)-1,2,3,4-tetrahydro-1-naphthalenone (128): Obtained as the exclusive isomer in 76\% yield from 127 using Procedure D in 45 min. Purified by flash chromatography (9:1 pentane:Et $\left.{ }_{2} \mathrm{O}\right)$ to afford a clear colorless oil. ${ }^{1} \mathrm{H}$ NMR $\left(\mathrm{CDCl}_{3}, 300 \mathrm{MHz}\right) \delta 8.02(\mathrm{~d}, J=7.8 \mathrm{~Hz}, 1 \mathrm{H}), 7.44(\mathrm{dt}, J=7.5,1.3$ $\mathrm{Hz}, 1 \mathrm{H}), 7.31-7.16(\mathrm{~m}, 7 \mathrm{H}), 2.98-2.95(\mathrm{~m}, 2 \mathrm{H}), 2.66(\mathrm{dt}, J=7.7,2.4 \mathrm{~Hz}, 2 \mathrm{H}), 2.53-2.44(\mathrm{~m}, 1 \mathrm{H}), 2.22$ (dq, $J=13.3,4.6 \mathrm{~Hz}, 1 \mathrm{H}), 2.06-1.81(\mathrm{~m}, 2 \mathrm{H}), 1.79-1.67(\mathrm{~m}, 2 \mathrm{H}), 1.61-1.49(\mathrm{~m}, 1 \mathrm{H}) ;{ }^{13} \mathrm{C} \mathrm{NMR}\left(\mathrm{CDCl}_{3}, 75\right.$ MHz) $\delta 200.2,143.9,142.4,133.1,132.5,128.6,128.4,128.3,127.4,126.5,125.7,47.4,36.1,29.2,28.9$, 28.3, 28.2; $\mathrm{HRMS}(\mathrm{EI}) \mathrm{m} / z$ calcd for $\mathrm{C}_{19} \mathrm{H}_{20} \mathrm{O}\left(\mathrm{M}^{+}\right)$: 264.1514. Found: 264.1509.

5-(1,2-Diphenylethyl)-2,2-dimethyl-1,3-dioxane-4,6-dione (129): Phenyl Meldrum's alkylidene ${ }^{3}$ was prepared by the conjugate addition of phenylmagnesium bromide to 5-methoxymethylene Meldrum's $\operatorname{acid}^{34}$ according to Procedure $\mathrm{C}$, followed by acid hydrolysis with $10 \% \mathrm{HCl}$ for 30 minutes. After standard workup the phenyl Meldrum's alkylidene was acquired as a pale yellow solid in quantitative yield. This was reacted with benzylmagnesium chloride (Procedure C) to produce the desired target material, which was purified by flash chromatography $\left(1: 1\right.$ hexanes: $\left.\mathrm{CH}_{2} \mathrm{Cl}_{2}\right)$ to afford a colorless oil in $82 \%$ yield. ${ }^{1} \mathrm{H}$ $\operatorname{NMR}\left(\mathrm{CDCl}_{3}, 300 \mathrm{MHz}\right)$ d 7.39-7.20 (m, 10H), 4.10-4.03 (m, 1H), $3.70(\mathrm{t}, J=12.4 \mathrm{~Hz}, 1 \mathrm{H}), 3.53(\mathrm{~d}, J=$ $2.8 \mathrm{~Hz}, 1 \mathrm{H}), 3.23(\mathrm{dd}, J=13.7,5.7 \mathrm{~Hz}, 1 \mathrm{H}), 1.52(\mathrm{~s}, 3 \mathrm{H}), 1.07(\mathrm{~s}, 3 \mathrm{H}) ;{ }^{13} \mathrm{C} \mathrm{NMR}\left(\mathrm{CDCl}_{3}, 75 \mathrm{MHz}\right) \delta$ 166.0, 164.5, 139.5, 139.0, 129.3, 128.9, 128.7, 127.8, 126.7, 105.3, 48.4, 47.7, 38.1, 28.0; HRMS(EI) $\mathrm{m} / z$ calcd for $\mathrm{C}_{17} \mathrm{H}_{14} \mathrm{O}_{3}\left(\mathrm{M}^{+}-\mathrm{C}_{3} \mathrm{H}_{6} \mathrm{O}\right)$ : 266.0943. Found: 266.0933 . 
3-Phenyl-1,2,3,4-tetrahydro-1-naphthalenone (130): Obtained as the exclusive isomer in 51\% yield from 129 using Procedure D with slow addition of substrate by syringe pump over $8 \mathrm{~h}$, followed by an additional one hour of stirring at $100{ }^{\circ} \mathrm{C}$. Purified by flash chromatography $\left(1: 1\right.$ hexanes: $\left.\mathrm{CH}_{2} \mathrm{Cl}_{2}\right)$ to afford a colorless oil. ${ }^{1} \mathrm{H}$ NMR $\left(\mathrm{CDCl}_{3}, 300 \mathrm{MHz}\right) \delta 8.07(\mathrm{~d}, J=7.8 \mathrm{~Hz}, 1 \mathrm{H}), 7.50(\mathrm{t}, J=7.4 \mathrm{~Hz}, 1 \mathrm{H}), 7.39-7.24$ (m, 7H), 3.51-3.40 (m, 1H), 3.21-3.17 (m, 2H), $2.97(\mathrm{dd}, J=16.7,3.5 \mathrm{~Hz}, 1 \mathrm{H}), 2.83(\mathrm{dd}, J=16.6,12.9 \mathrm{~Hz}$ $1 \mathrm{H}) ;{ }^{13} \mathrm{C} \mathrm{NMR}\left(\mathrm{CDCl}_{3}, 75 \mathrm{MHz}\right) \delta 197.8,143.4,133.8,132.1,128.8,127.2,127.0,126.9,126.7,45.9$, 41.1, 37.7; $\mathrm{HRMS}(\mathrm{EI}) \mathrm{m} / z$ calcd for $\mathrm{C}_{16} \mathrm{H}_{14} \mathrm{O}\left(\mathrm{M}^{+}\right)$: 222.1045. Found: 222.1044 .

5-(1,3-Diphenylpropyl)-2,2-dimethyl-1,3-dioxane-4,6-dione (131): Cinnamaldehyde was condensed with Meldrum's acid using the method of Bigi ${ }^{9}$, and the resulting alkylidene reacted with phenylmagnesium bromide according to Procedure $\mathrm{C}$. The resulting product was hydrogenated over $5 \%$ $\mathrm{Pd} / \mathrm{C}$ to afford the target material as a solid in 56\% yield (3 steps from Meldrum's acid) after recrystallization from MeOH. M.p. 72.5-73.5 ${ }^{\circ} \mathrm{C}(\mathrm{MeOH}) ;{ }^{1} \mathrm{H}$ NMR $\left(\mathrm{CDCl}_{3}, 300 \mathrm{MHz}\right) \delta$ 7.35-7.12 (m, $10 \mathrm{H}), 3.80-3.74(\mathrm{~m}, 1 \mathrm{H}), 3.67(\mathrm{~d}, J=3.2 \mathrm{~Hz}, 1 \mathrm{H}), 2.73-2.51(\mathrm{~m}, 3 \mathrm{H}), 2.36-2.24(\mathrm{~m}, 1 \mathrm{H}), 1.59(\mathrm{~s}, 3 \mathrm{H})$, $1.11(\mathrm{~s}, 3 \mathrm{H}) ;{ }^{13} \mathrm{C} \mathrm{NMR}\left(\mathrm{CDCl}_{3}, 75 \mathrm{MHz}\right) \delta 165.5,164.6,141.3,139.0,129.1,128.8,128.4,127.8,126.0$, 105.4, 51.4, 45.5, 34.3, 34.1, 28.3, 28.0; HRMS(EI) $m / z$ calcd for $\mathrm{C}_{21} \mathrm{H}_{22} \mathrm{O}_{4}\left(\mathrm{M}^{+}\right)$: 338.1518. Found: 338.1519.

7-Phenyl-6,7,8,9-tetrahydro-5H-benzo[a]cyclohepten-5-one (132): Obtained as the exclusive isomer in $42 \%$ yield from 131 using Procedure D with slow addition of substrate by syringe pump over 8 $\mathrm{h}$, followed by an additional one hour of stirring at $100{ }^{\circ} \mathrm{C}$. Purified by flash chromatography $(1: 1$ hexanes: $\left.\mathrm{CH}_{2} \mathrm{Cl}_{2}\right)$ to afford a colorless oil. ${ }^{1} \mathrm{H} \mathrm{NMR}\left(\mathrm{CDCl}_{3}, 300 \mathrm{MHz}\right) \delta 7.73(\mathrm{~d}, J=7.6 \mathrm{~Hz}, 1 \mathrm{H}), 7.59(\mathrm{t}$, $J=7.4 \mathrm{~Hz}, 1 \mathrm{H}), 7.49(\mathrm{~d}, J=7.7 \mathrm{~Hz}, 1 \mathrm{H}), 7.36(\mathrm{t}, J=7.4 \mathrm{~Hz}, 1 \mathrm{H}), 7.31-7.17(\mathrm{~m}, 5 \mathrm{H}), 3.40-3.34(\mathrm{~m}, 1 \mathrm{H})$, $2.89(\mathrm{dd}, J=19.0,7.5 \mathrm{~Hz}, 1 \mathrm{H}), 2.75-2.68(\mathrm{~m}, 2 \mathrm{H}), 2.43(\mathrm{dd}, J=19.0,3.3 \mathrm{~Hz}, 1 \mathrm{H}), 2.32-2.20(\mathrm{~m}, 1 \mathrm{H})$, 1.86-1.74 (m, 1H); ${ }^{13} \mathrm{C} \mathrm{NMR}\left(\mathrm{CDCl}_{3}, 75 \mathrm{MHz}\right) \delta 158.5,141.4,136.8,134.7,128.5,128.3,127.6,126.1$, 125.5, 123.6, 43.0, 37.8, 37.7, 33.9; $\mathrm{HRMS}(\mathrm{EI}) \mathrm{m} / \mathrm{z}$ calcd for $\mathrm{C}_{17} \mathrm{H}_{16} \mathrm{O}\left(\mathrm{M}^{+}\right):$236.1201. Found: 236.1194.

5-(1-Benzyl-3-phenylpropyl)-2,2-dimethyl-1,3-dioxane-4,6-dione (133): Cinnamaldehyde was condensed with Meldrum's acid ${ }^{9}$, and the resulting alkylidene reacted with benzylmagnesium chloride according to Procedure $\mathrm{C}$. The resulting product was hydrogenated over $5 \% \mathrm{Pd} / \mathrm{C}$ to afford the target material as a solid in 65\% yield (3 steps from Meldrum's acid) after recrystallization from MeOH. M.p. 83.0-84.0 ${ }^{\circ} \mathrm{C}(\mathrm{MeOH}) ;{ }^{1} \mathrm{H}$ NMR $\left(\mathrm{CDCl}_{3}, 300 \mathrm{MHz}\right) \delta$ 7.31-7.14 (m, 10H), $3.29(\mathrm{~d}, J=2.4 \mathrm{~Hz}, 1 \mathrm{H}), 2.98$ 
$(\mathrm{dd}, J=8.1,2.3 \mathrm{~Hz}, 2 \mathrm{H}), 2.87-2.74(\mathrm{~m}, 2 \mathrm{H}), 2.65-2.55(\mathrm{~m}, 1 \mathrm{H}), 1.98-1.84(\mathrm{~m}, 2 \mathrm{H}), 1.66(\mathrm{~s}, 3 \mathrm{H}), 1.51(\mathrm{~s}$, $3 \mathrm{H}) ;{ }^{13} \mathrm{C} \mathrm{NMR}\left(\mathrm{CDCl}_{3}, 75 \mathrm{MHz}\right) \delta 165.5,164.7,141.5,139.6,129.2,128.7,128.5,128.4,126.7,126.0$, 104.6, 47.4, 40.6, 37.4, 34.3, 32.7, 28.2, 26.7; $\mathrm{HRMS}(\mathrm{EI}) \mathrm{m} / \mathrm{z}$ calcd for $\mathrm{C}_{19} \mathrm{H}_{18} \mathrm{O}_{3}\left(\mathrm{M}^{+}-\mathrm{C}_{3} \mathrm{H}_{6} \mathrm{O}\right)$ : 294.1256. Found: 294.1260.

3-Phenethyl-1,2,3,4-tetrahydro-1-naphthalenone (134): Obtained as the exclusive isomer in 59\% yield from 133 using Procedure D with slow addition of substrate by syringe pump over $8 \mathrm{~h}$, followed by an additional one hour of stirring at $100{ }^{\circ} \mathrm{C}$. Purified by flash chromatography $\left(1: 1\right.$ hexanes: $\left.\mathrm{CH}_{2} \mathrm{Cl}_{2}\right)$ to afford a colorless oil. ${ }^{1} \mathrm{H} \mathrm{NMR}\left(\mathrm{CDCl}_{3}, 300 \mathrm{MHz}\right) \delta 8.02(\mathrm{~d}, J=7.8 \mathrm{~Hz}, 1 \mathrm{H}), 7.47(\mathrm{t}, J=7.5 \mathrm{~Hz}, 1 \mathrm{H})$, 7.32-7.18 (m, 7H), $3.05(\mathrm{~d}, J=14.0 \mathrm{~Hz}, 1 \mathrm{H}), 2.86-2.79(\mathrm{~m}, 1 \mathrm{H}), 2.72(\mathrm{t}, J=7.9 \mathrm{~Hz}, 2 \mathrm{H}), 2.37(\mathrm{dd}, J=$ 16.2, $11.9 \mathrm{~Hz}, 1 \mathrm{H}), 2.27-2.19(\mathrm{~m}, 1 \mathrm{H}), 1.78$ (quintet, $J=7.6 \mathrm{~Hz}, 2 \mathrm{H}) ;{ }^{13} \mathrm{C} \mathrm{NMR}\left(\mathrm{CDCl}_{3}, 75 \mathrm{MHz}\right) \delta$ $198.2,143.5,141.7,133.5,132.3,128.9,128.4,128.3,126.9,126.7,125.9,45.2,37.4,36.1,34.7,32.8$; HRMS(EI) $m / z$ calcd for $\mathrm{C}_{18} \mathrm{H}_{18} \mathrm{O}\left(\mathrm{M}^{+}\right): 250.1358$. Found: 250.1366 .

\section{REFERENCES}

${ }^{1}$ For the synthesis of methyl alkylidene Meldrum's acid, see: Ziegler, F. E.; Guenther, T.; Nelson, R. V. Synth. Commun. 1980, 10, 661-665.

${ }^{2}$ Vogt, P. F.; Molino, B. F.; Robichaud, A. J. Synth. Commun. 2001, 31, 679-684.

${ }^{3}$ Baty, J. D.; Jones, G.; Moore, C. J. Org. Chem. 1969, 34, 3295-3302.

${ }^{4}$ (a) Marquardt, F.-H. Helv. Chim. Acta. 1965, 48, 1490-1493. (b) Johnson, W. S.; Glenn, H. J. J. Am. Chem. Soc. 1949, 71, 1092-1096.

${ }^{5}$ Huisgen, R.; Seidl, G.; Wimmer, I. Justus Liebigs Ann. Chem. 1964, 677, 21-33.

${ }^{6}$ Kasturi, T. R.; Abraham, E. M.; Prasad, R. S. Tetrahedron 1974, 30, 2887-2890.

${ }^{7}$ Kadesch, R. G. J. Am. Chem. Soc. 1944, 66, 1207-1213.

${ }^{8}$ Tóth, G.; Kövér, K. E. Synth. Commun. 1995, 25, 3067-3074. 
${ }^{9}$ Bigi, F.; Carloni, S.; Ferrari, L.; Maggi, R.; Mazzacani, A.; Sartori, G. Tetrahedron Lett. 2001, 42, 5203-5205.

${ }^{10}$ Posternak, T.; Castro, R. Helv. Chim. Acta. 1948, 31, 536-42.

${ }^{11}$ Huffman, J. W.; Opliger, C. E. J. Org. Chem. 1971, 36, 111-117.

${ }^{12}$ Levitz, M.; Perlman, D.; Bogert, M. T. J. Org. Chem. 1941, 6, 105-119.

${ }^{13}$ Brown, R. F. C.; Coulston, K. J.; Eastwood, F. W.; Gatehouse, B. M.; Guddatt, L. W.; Luke, W.; Pfenninger, M.; Rainbow, I. Aust. J. Chem. 1984, 37, 2509-2524.

${ }^{14}$ Minami, S.; Tomita, M.; Takamatsu, H.; Uyeo, S. Chem. Pharm. Bull. 1965, 13, 1084-1091.

${ }^{15}$ House, H.; Schellenbaum, M. J. Org. Chem. 1963, 28, 34-38.

${ }^{16}$ Carter, R. H.; Colyer, R. M.; Hill, R. A.; Staunton, J. J. Chem. Soc., Perkin Trans. 1 1976, 14381441.

${ }^{17}$ Layer, R. W.; MacGregor, I. R. J. Org. Chem. 1956, 21, 1120-1123.

${ }^{18}$ Schrecker, A. W.; Hartwell, J. L. J. Am. Chem. Soc. 1957, 79, 3827-3830.

${ }^{19}$ Scheuer, P. J.; Cohen, S. G. J. Am. Chem. Soc. 1958, 80, 4933-4938.

${ }^{20}$ Torrado, A.; Imperiali, B. J. Org. Chem. 1996, 61, 8940-8948.

${ }^{21}$ Lednicer, D.; Babcock, J. C.; Marlatt, P. E.; Lyster, S. C.; Duncan, G. W. J. Med. Chem. 1965, 8, 5257.

${ }^{22}$ Buckley, III, T. F.; Rapoport, H. J. Am. Chem. Soc. 1980, 102, 3056-3062 and references cited therein.

${ }^{23}$ Carter, R. H.; Garson, M. J.; Hill, R. A.; Staunton, J.; Sunter, D. C. J. Chem. Soc., Perkin Trans. 1 1981, 471-479.

${ }^{24}$ Kometani, T.; Yoshii, E. J. Chem. Soc., Perkin Trans. 1 1981, 1191-1196. 
${ }^{25}$ Cardozo, M.G.; Iimura, Y.; Sugimoto, H.; Yamanishi, Y.; Hopfinger, A. J. J. Med. Chem. 1992, 35, 584-589.

${ }^{26}$ Chan, C.-C.; Huang, X. Synthesis 1982, 452-454.

${ }^{27}$ Diouf, O.; Depreux, P.; Chavatte, P.; Poupaert, J. H. Eur. J. Med. Chem. 2000, 35, 699-706.

${ }^{28}$ Sam, J.; Alwani, D. W.; Aparajithan, K. J. Heterocycl. Chem. 1965, 2, 366-369.

${ }^{29}$ Hin, B.; Majer, P.; Tsukamoto, T. J. Org. Chem. 2002, 67, 7365-7368 and references cited therein.

${ }^{30}$ Adam, R. T.; Geissman, T. A.; Baker, B. R.; Teeter, H. M. J. Am. Chem. Soc. 1941, 62, 528-532.

${ }^{31}$ Borsche, W.; Niemann, J. Justus Liebigs Ann. Chem. 1933, 502, 264-268.

${ }^{32}$ Caunt, D.; Crow, W. D.; Haworth, R. D.; Vodoz, C. A. J. Chem. Soc. 1950, 1631-1635.

${ }^{33}$ Borsche, W.; Hofmann; K. Justus Liebigs Ann. Chem. 1943, 554, $23-27$.

${ }^{34}$ Jourdain, F.; Pommelet, J. C. Synth. Commun. 1997, 27, 483-492. 